\title{
25. QUATERNARY AND PLIOCENE PLANKTONIC FORAMINIFERS OF THE NORTHEASTERN ATLANTIC (GOBAN SPUR), DEEP SEA DRILLING PROJECT LEG 80
}

\author{
Claude Pujol and Josette Duprat, Département de Géologie et Océanographie, Université de Bordeaux I²
}

\begin{abstract}
Quaternary to Pliocene planktonic foraminifers of the Goban Spur, off the Irish coast, were investigated for their biostratigraphic and environmental significance.

Three biostratigraphic zones were recognized in the Pliocene. The uppermost of these (the Globorotalia inflata Zone) includes the transition between the Pliocene and the Quaternary. The Quaternary (the $G$. truncatulinoides Zone) is subdivided into three subzones; from the base, the $G$. truncatulinoides, G. hirsuta, and Turborotalita humilis subzones. The thorough study of ecological assemblages of planktonic foraminifers led to the division of the PlioceneQuaternary sequence into some 30 bioclimatic subdivisions. Some of them are correlated with isotopic stages.

The Pliocene/Quaternary boundary does not appear to be well marked in the foraminiferal assemblages. The first indication of cooling appears at the base of the Olduvai Event. The first appearance datum (FAD) of G. truncatulinoides, which is usually used as a marker of the Quaternary base, occurs at the Jaramillo Event.

A significant biological event, the first appearance of $G$. hirsuta, is dated as occurring approximately 470,000 yr. ago and marks an environmental change. It occurs during bioclimatic Subdivision XIII.

The surficial Quaternary paleoenvironments in the North Atlantic were studied. The hydrological environment is illustrated by estimates of summer and winter surface water temperatures. During glacial periods, water temperatures are assumed to have averaged approximately $5.8^{\circ} \mathrm{C}$ in summer and $0^{\circ} \mathrm{C}$ in winter. All year long, there is a difference of about $10^{\circ} \mathrm{C}$ between the present and glacial periods that is also characteristic of the changes in geographic regions that are subject to intermittent glaciation. The general pattern of temperature estimates shows that a slight general cooling has taken place from 470,000 yr. ago to the present.

Twenty-four species of planktonic foraminifers were identified. Some of the most abundant of these are illustrated.
\end{abstract}

\section{INTRODUCTION}

Quaternary sediments were recovered from three holes (Holes 548, 549A, and 550) that were drilled during Leg 80 in the northeastern Atlantic Ocean (Table 1, Fig. 1).

These holes are located on a transect of the Goban Spur, a structural high in the Variscan basement that is blanketed by a relatively thin sedimentary cover (see site chapters, this volume) and follows an ENE-WSW trend on the northwest European Atlantic continental margin north of the Bay of Biscay.

The Quaternary sediments in Holes 550 and 549A are marly calcareous foraminifer nannofossil oozes (Lithologic Unit 1, site chapters, this volume). The upper Qua-

Table 1. Quaternary data for Leg 80 holes.

\begin{tabular}{lccccc}
\hline Hole & $\begin{array}{c}\text { Latitude } \\
(\mathrm{N})\end{array}$ & $\begin{array}{c}\text { Longitude } \\
(\mathrm{W})\end{array}$ & $\begin{array}{c}\text { Water depth } \\
(\mathrm{m})\end{array}$ & $\begin{array}{c}\text { Depth of } \\
\text { cored section } \\
(\mathrm{m})\end{array}$ & $\begin{array}{c}\text { Number of } \\
\text { samples }\end{array}$ \\
\hline 548 & $48^{\circ} 54^{\prime} 95$ & $12^{\circ} 09^{\prime} 84$ & 1256 & $110\left(210^{\mathrm{a}}\right)$ & $147\left(217^{\mathrm{b}}\right)$ \\
$549 \mathrm{~A}$ & $49^{\circ} 05^{\prime} 29$ & $13^{\circ} 05^{\prime} 89$ & 2535.5 & 27 & 52 \\
550 & $48^{\circ} 30^{\prime} 91$ & $13^{\circ} 26^{\prime} 37$ & 4432 & 5.5 & 10 \\
\hline
\end{tabular}

a Includes Pliocene section.

b Includes samples from Pliocene section and samples used for biostratigraphic investigation.

\footnotetext{
${ }^{1}$ Graciansky, P. C. de, Poag, C. W., et al., Init. Repts. DSDP, 80: Washington (U.S. Govt, Printing Office).

2 Address: Département de Géologie et Océanographie, Avenue des Facultés, 33405 Talence Cedex, France.
}

ternary sediments in Hole 548 are of the same type, but the lower Pleistocene and Pliocene sediments are reddish to grayish clay and nannofossil oozes (Lithologic Units 2 and 3 , site chapters, this volume). The planktonic foraminifers in the three holes are generally abundant and well preserved. They are seldom heavily damaged or broken, although they are affected to varying degrees by dissolution.

The purpose of this study is twofold. First, we wanted to recognize and define the biostratigraphic framework of the whole Quaternary sequence. We extended our observations several meters into Pliocene sediments in order to establish accurate biostratigraphic reference points for the correlation of the Pliocene/Quaternary boundary. Second, we wanted to determine within the Quaternary sequence the chief stages of climatic change and to estimate the temperature of surface water for summer and winter during these stages.

\section{MATERIAL AND METHODS}

Sampling that was random (with regard to the lithology) was carried out in the Hole 548 and 549A sediments; however, at least one sample was acquired per section. Because of the relatively high accumulation rate in the late Quaternary sediments in Hole 548 and the low accumulation rate at the base of the Quaternary sequence in Hole 549A (site chapters, this volume), we chose to consider the holes as complementary and used both to reconstruct a continuous and complete record of the Quaternary. It was possible to observe the biostratigraphic evolution of planktonic microfauna from the Pliocene upward in Hole 548 (Cores 35-16). In Hole 550 the Quaternary sequence recovered was short; it consisted of one core only.

Each sample (initial volume: $5-10 \mathrm{~cm}^{3}$ ) was used to evaluate carbonate content. The samples were washed through standard sieves and 

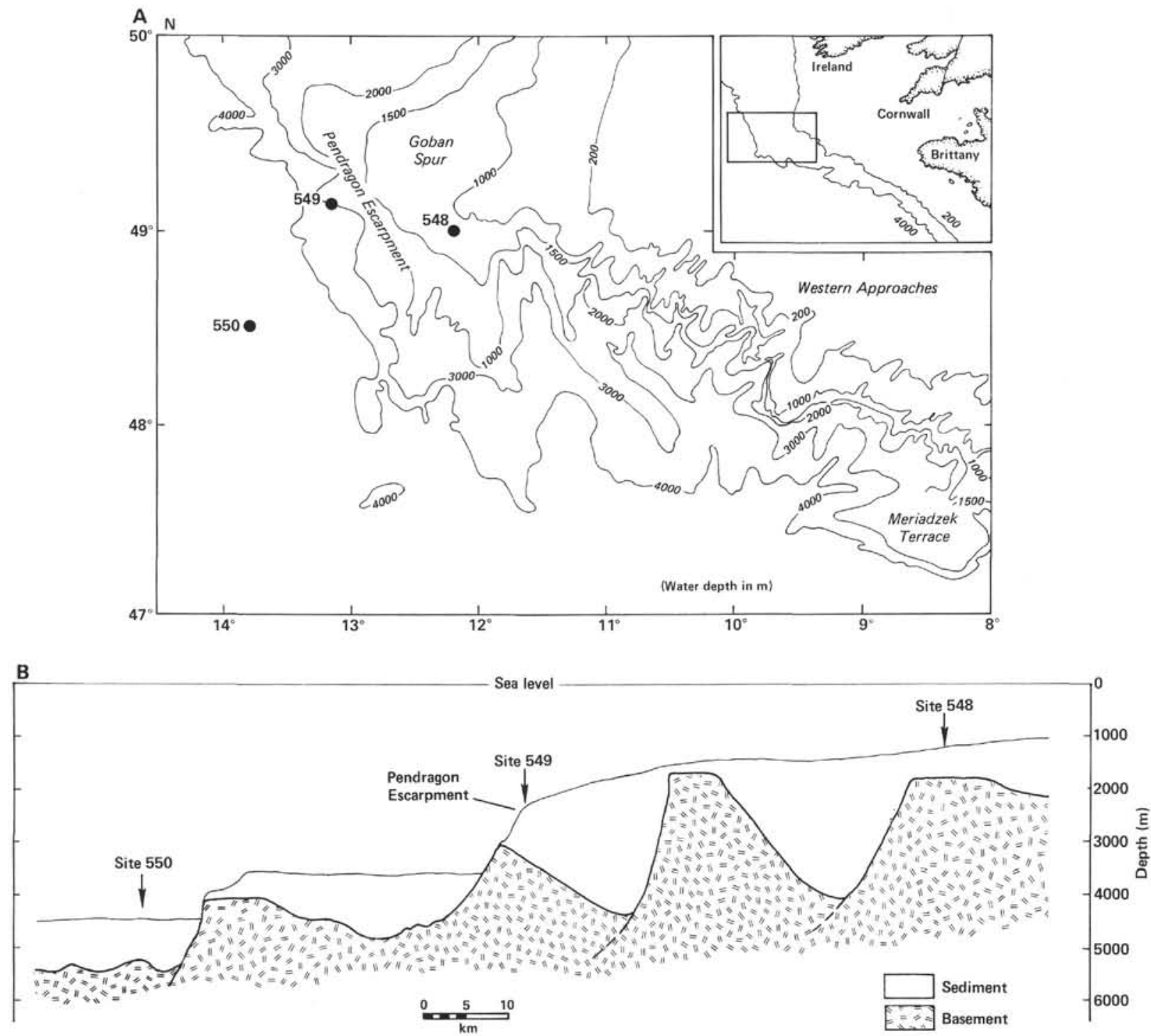

Figure 1. Location of Sites 548,549 , and 550. A. Position with respect to bathymetry. B. Position with respect to topography and sediment thickness. Quaternary sediment depths are $5.5 \mathrm{~m}$ at Site $550,27 \mathrm{~m}$ at Site 549, and $101 \mathrm{~m}$ at Site 548.

weighed; the fraction sizes were $>150 \mu \mathrm{m}, 150$ to $63 \mu \mathrm{m}$, and $<63$ $\mu \mathrm{m}$.

Planktonic microfauna could be identified in the two larger size fractions. Abundances were established from the $>150 \mu \mathrm{m}$ size fraction; they are based on no fewer than about 300 specimens.

The distribution of the different species in each hole is summarized in Tables 2 to 4 . The abundance, preservation, and dissolution of the planktonic microfauna are plotted for each sample.

\section{REGIONAL FAUNAL ASSOCIATION}

The present-day biocoenosis within the area investigated is an assemblage characteristic of temperate areas, and we have already determined the following specific proportions (Pujol, 1980); Globigerina pachyderma (leftcoiling), $1.5 \%$; G. pachyderma (right-coiling), $29 \%$; G. bulloides, $36 \%$; G. quinqueloba, 10\%; Globorotalia scitula, 2\%; Globigerinita glutinata, 13\%; Globorotalia inflata, 7\%; Orbulina universa, $1 \%$; Hastigerina siphonifera, $<1 \%$. In addition, there are a few specimens of Turborotalita humilis, G. hirsuta, and G. truncatulinoides. The authors identified this microfauna as belonging to the biogeographic area of Globigerina pachyderma (right-coiling). It corresponds to a subdivision of the subarctic and transitional assemblages recognized by Bé (1977).

The faunal assemblage observed in surficial marine sediments (recent thanatocoenosis) is made up of the same species.

The Quaternary species recognized on the Goban Spur are as follows: arctic fauna-G. pachyderma (left-coiling); subarctic fauna-G. pachyderma (right-coiling), G. bulloides, G. quinqueloba, Globigerinita uvula; transitional or winter subtropical fauna-Globorotalia scitula, G. inflata, Globigerinita glutinata, O. universa, $H$. siphonifera, Globorotalia truncatulinoides, G. hirsuta, T. humilis; summer subtropical fauna-Globigerinoides ruber, Globorotalia crassaformis div. sp., Globigerinoides conglobatus, Globigerina rubescens, G. falconensis, G. calida; tropical fauna-Globigerinoides trilobus, $G$. sacculiferus, Globorotalia menardii, Sphaeroidinella dehiscens, Globigerina digitata. 
Table 2A. Distribution of planktonic foraminifers in Hole 548 (Cores 1-5).

\begin{tabular}{|c|c|c|c|c|c|c|c|c|c|c|c|c|c|c|c|c|c|c|c|c|}
\hline 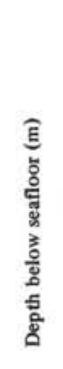 & 今ั & 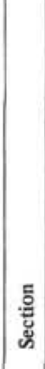 & 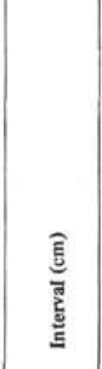 & 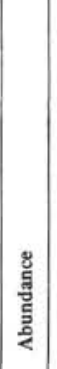 & 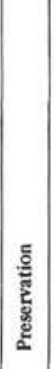 & 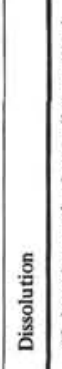 & 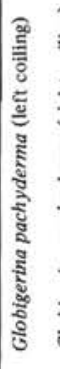 & 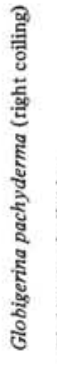 & 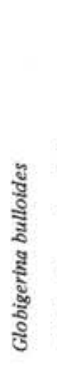 & 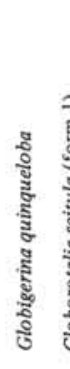 & 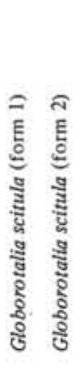 & 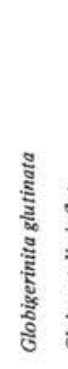 & 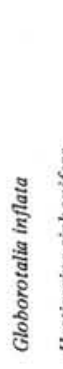 & 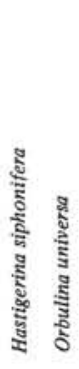 & 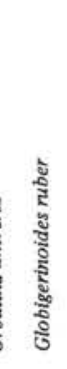 & 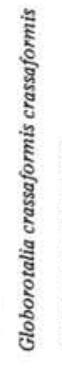 & 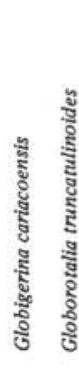 & 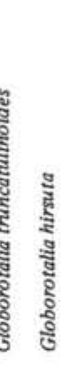 & 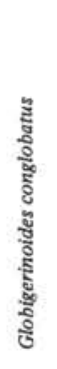 & 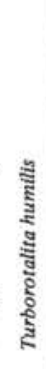 \\
\hline 4. & 1 & 2 & $\begin{array}{c}0-3 \\
4-5 \\
14-17 \\
70-71 \\
40-43 \\
70-71 \\
90 \cdot-93 \\
20.23 \\
\end{array}$ & \begin{tabular}{|l|}
$\mathrm{A}$ \\
$\mathrm{A}$ \\
$\mathrm{A}$ \\
$\mathrm{C}$ \\
$\mathrm{R}$ \\
$\mathrm{F}$ \\
$\mathrm{R}$ \\
$\mathrm{R}$ \\
\end{tabular} & \begin{tabular}{|l|}
$\mathrm{G}$ \\
$\mathrm{G}$ \\
$\mathrm{G}$ \\
$\mathrm{M}$ \\
$\mathrm{G}$ \\
$\mathrm{G}$ \\
$\mathrm{G}$ \\
$\mathrm{G}$ \\
\end{tabular} & \begin{tabular}{l|l}
2 \\
2 \\
2 \\
\\
1 \\
1 \\
1 \\
1 \\
\end{tabular} & \begin{tabular}{l|l}
$\mathrm{T}$ & $\mathrm{T}$ \\
$\mathrm{T}$ & \\
$\mathrm{R}$ & \\
$\mathrm{P}$ & \\
$\mathrm{C}$ & \\
$\mathrm{A}$ & \\
$\mathrm{A}$ & \\
$\mathrm{A}$ & \\
\end{tabular} & \begin{tabular}{l|l}
$\mathrm{C}$ & \\
$\mathrm{P}$ & \\
$\mathrm{C}$ & \\
$\mathrm{C}$ & \\
& \\
& \\
$\mathrm{T}$ & \\
$\mathrm{T}$ &
\end{tabular} & $\begin{array}{l}\mathrm{P} \\
\mathrm{P} \\
\mathrm{C} \\
\mathrm{C} \\
\mathrm{C} \\
\mathrm{T} \\
\mathrm{T}\end{array}$ & \begin{tabular}{|l|l}
$\mathrm{P}$ & $\mathrm{R}$ \\
$\mathrm{P}$ & $\mathrm{R}$ \\
$\mathrm{P}$ & $\mathrm{R}$ \\
$\mathrm{P}$ & $\mathrm{R}$ \\
$\mathrm{R}$ & $\mathrm{R}$ \\
$\mathrm{T}$ & \\
\end{tabular} & $\begin{array}{l}\mathrm{R} \\
\mathrm{R} \\
\mathrm{R} \\
\mathrm{R} \\
\mathrm{R}\end{array}$ & \begin{tabular}{|l|l} 
R & R \\
P & \\
R & \\
\end{tabular} & \begin{tabular}{l|l}
$\mathrm{P}$ & $\mathrm{P}$ \\
$\mathrm{R}$ & $\mathrm{F}$ \\
$\mathrm{P}$ & $\mathrm{F}$ \\
$\mathrm{P}$ & \\
$\mathrm{T}$ &
\end{tabular} & \begin{tabular}{l|l}
$R$ & $R$ \\
$R$ & $R$ \\
$R$ & $R$ \\
& $R$
\end{tabular} & $\begin{array}{l}\mathrm{R} \\
\mathrm{R} \\
\mathrm{R}\end{array}$ & & $\begin{array}{l}\mathbf{R} \\
\mathbf{R} \\
\mathbf{R} \\
\mathbf{R}\end{array}$ & \begin{tabular}{l|l}
$\mathrm{R}$ \\
$\mathrm{R}$ \\
$\mathrm{R}$ \\
$\mathrm{R}$
\end{tabular} & & P \\
\hline 1 & 2 & $\begin{array}{l}1 \\
2 \\
3 \\
4 \\
5\end{array}$ & $\begin{array}{c}20.23 \\
41-42 \\
20-23 \\
20-23 \\
33-36 \\
53-54 \\
2-3 \\
33-36 \\
70-71 \\
33-36 \\
33-36\end{array}$ & \begin{tabular}{|l|} 
F \\
C \\
A \\
A \\
A \\
F \\
C \\
C \\
R \\
C \\
C \\
\end{tabular} & \begin{tabular}{|l|}
$\mathrm{G}$ \\
$\mathrm{G}$ \\
$\mathrm{G}$ \\
$\mathrm{M}$ \\
$\mathrm{M}$ \\
$\mathrm{G}$ \\
$\mathrm{G}$ \\
$\mathrm{G}$ \\
$\mathrm{G}$ \\
$\mathrm{M}$ \\
$\mathrm{G}$ \\
\end{tabular} & \begin{tabular}{|l|l|}
1 & \\
2 & \\
2 & \\
2 & \\
2 & \\
1 & \\
2 & \\
2 & \\
1 & \\
2 & \\
\end{tabular} & \begin{tabular}{|l|l}
$A$ & \\
$C$ & \\
$R$ & \\
$P$ & \\
$P$ & \\
$P$ & \\
$A$ & \\
& $A$ \\
R & \\
\end{tabular} & \begin{tabular}{l|l}
$\mathrm{R}$ & \\
$\mathrm{P}$ & \\
$\mathrm{C}$ & \\
$\mathrm{C}$ & \\
$\mathrm{C}$ & \\
$\mathrm{P}$ & \\
$\mathrm{P}$ & \\
$\mathrm{P}$ & \\
$\mathrm{R}$ & \\
$\mathrm{C}$ & \\
$\mathrm{A}$ &
\end{tabular} & \begin{tabular}{l|l}
$\mathrm{R}$ \\
$\mathrm{C}$ \\
$\mathrm{C}$ \\
$\mathrm{A}$ \\
$\mathrm{A}$ \\
$\mathrm{C}$ \\
$\mathrm{P}$ \\
$\mathrm{A}$ \\
$\mathrm{R}$ \\
$\mathrm{A}$ \\
$\mathrm{C}$
\end{tabular} & \begin{tabular}{|l|l}
$\mathrm{R}$ & $\mathrm{R}$ \\
$\mathrm{R}$ & $\mathrm{R}$ \\
$\mathrm{P}$ & $\mathrm{P}$ \\
$\mathrm{P}$ & $\mathrm{R}$ \\
$\mathrm{P}$ & $\mathrm{R}$ \\
$\mathrm{R}$ & $\mathrm{R}$ \\
$\mathrm{R}$ & $\mathrm{R}$ \\
$\mathrm{P}$ & $\mathrm{R}$ \\
& \\
$\mathrm{P}$ & $\mathrm{R}$ \\
$\mathrm{P}$ & $\mathrm{R}$ \\
\end{tabular} & $\begin{array}{l}\mathrm{R} \\
\mathrm{R} \\
\mathrm{P} \\
\mathrm{R} \\
\mathrm{R} \\
\mathrm{R} \\
\mathrm{R} \\
\mathrm{R} \\
\mathrm{R} \\
\mathrm{R} \\
\mathrm{R}\end{array}$ & \begin{tabular}{|l}
$\mathrm{R}$ \\
$\mathrm{R}$ \\
$\mathrm{R}$ \\
$\mathrm{R}$ \\
$\mathrm{R}$ \\
$\mathrm{R}$ \\
$\mathrm{R}$ \\
$\mathrm{P}$
\end{tabular} & $\begin{array}{l}R \\
P \\
R \\
R \\
R \\
T \\
R \\
R \\
R \\
R\end{array}$ & $\begin{array}{l}\mathbf{R} \\
\mathbf{R} \\
\end{array}$ & & & R & $\mathbf{R}$ & & \\
\hline 23.0 & 3 & \begin{tabular}{|c|}
2 \\
3 \\
4 \\
5 \\
6 \\
7 \\
$\mathrm{CC}$ \\
\end{tabular} & \begin{tabular}{|c|}
$34-35$ \\
$56-57$ \\
$76-79$ \\
$113-120$ \\
$76-79$ \\
$76-79$ \\
$76-79$ \\
$76-79$ \\
$76-79$ \\
$29-32$
\end{tabular} & \begin{tabular}{|l|} 
C \\
A \\
A \\
A \\
A \\
A \\
C \\
F \\
C \\
C \\
A \\
\end{tabular} & \begin{tabular}{|l|} 
G \\
G \\
G \\
G \\
G \\
G \\
G \\
G \\
G \\
G \\
G \\
\end{tabular} & \begin{tabular}{|l|}
3 \\
2 \\
2 \\
2 \\
2 \\
1 \\
2 \\
1 \\
1 \\
1 \\
1 \\
\end{tabular} & 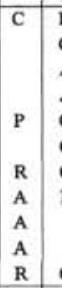 & \begin{tabular}{l|l}
$\mathrm{P}$ & \\
$\mathrm{C}$ & \\
$\mathrm{A}$ & \\
$\mathrm{A}$ & \\
$\mathrm{C}$ & \\
$\mathrm{C}$ & \\
$\mathrm{C}$ & \\
$\mathrm{R}$ & \\
& \\
$\mathrm{C}$ & \\
\end{tabular} & \begin{tabular}{l|}
$\mathrm{C}$ \\
$\mathrm{C}$ \\
$\mathrm{A}$ \\
$\mathrm{C}$ \\
$\mathrm{C}$ \\
$\mathrm{C}$ \\
$\mathrm{C}$ \\
$\mathrm{P}$ \\
$\mathrm{R}$ \\
$\mathrm{R}$ \\
$\mathrm{C}$
\end{tabular} & \begin{tabular}{|l|l}
$\mathrm{P}$ & $\mathrm{R}$ \\
$\mathrm{P}$ & $\mathrm{R}$ \\
$\mathrm{P}$ & $\mathrm{R}$ \\
$\mathrm{P}$ & $\mathrm{R}$ \\
$\mathrm{R}$ & $\mathrm{R}$ \\
$\mathrm{R}$ & $\mathrm{R}$ \\
& \\
& \\
$\mathrm{R}$ & \\
& $\mathrm{T}$ \\
$\mathrm{R}$ & $\mathrm{R}$ \\
\end{tabular} & $\begin{array}{l}\mathrm{R} \\
\mathrm{R} \\
\mathrm{R} \\
\mathrm{R} \\
\mathrm{R} \\
\mathrm{R}\end{array}$ & \begin{tabular}{|l|}
$R$ \\
$P$ \\
$P$ \\
$P$ \\
$R$ \\
$R$ \\
$R$ \\
$R$ \\
$R$ \\
$R$ \\
$R$ \\
\end{tabular} & \begin{tabular}{l|l}
$\mathrm{R}$ & \\
$\mathrm{P}$ & \\
$\mathrm{P}$ & \\
$\mathrm{P}$ & \\
$\mathrm{P}$ & \\
$\mathrm{P}$ & \\
$\mathrm{P}$ & \\
&
\end{tabular} & \begin{tabular}{l|l}
$\mathrm{T}$ & $\mathrm{P}$ \\
$\mathrm{R}$ \\
$\mathrm{R}$ & $\mathrm{R}$ \\
& $\mathrm{R}$ \\
& $\mathrm{R}$ \\
& $\mathrm{R}$
\end{tabular} & R & $\begin{array}{l}\mathrm{T} \\
\mathrm{R}\end{array}$ & $\begin{array}{l}\mathrm{R} \\
\mathrm{R} \\
\mathrm{R} \\
\mathrm{R} \\
\mathrm{R}\end{array}$ & \begin{tabular}{l|l} 
& $R$ \\
$R$ & $R$ \\
$R$ & $R$ \\
$R$ & $R$ \\
$R$ & $R$ \\
$R$ & $R$
\end{tabular} & $\mathbf{R}$ & \\
\hline 32.5 & 4 & $\begin{array}{l}1 \\
2 \\
3 \\
4 \\
5 \\
6 \\
7\end{array}$ & $\begin{array}{l}61-65 \\
61-65 \\
61-65 \\
61-65 \\
61-65 \\
20-23 \\
10-13 \\
\end{array}$ & \begin{tabular}{l|}
$\mathrm{A}$ \\
$\mathrm{A}$ \\
$\mathrm{C}$ \\
$\mathrm{A}$ \\
$\mathrm{C}$ \\
$\mathrm{C}$ \\
$\mathrm{C}$
\end{tabular} & \begin{tabular}{|l|}
$G$ \\
$G$ \\
$G$ \\
$G$ \\
$G$ \\
$G$ \\
$G$ \\
$G$ \\
\end{tabular} & $\begin{array}{l}2 \\
2 \\
2 \\
2 \\
1 \\
1 \\
1 \\
\end{array}$ & \begin{tabular}{l|l} 
& \\
& \\
P & \\
C & \\
R & \\
A & \\
\end{tabular} & \begin{tabular}{l|l}
$\mathrm{C}$ & \\
$\mathrm{A}$ & \\
$\mathrm{c}$ & \\
$\mathrm{C}$ & \\
$\mathrm{P}$ & \\
$\mathrm{C}$ & \\
$\mathrm{T}$ &
\end{tabular} & $\begin{array}{l}\text { C } \\
\text { C } \\
\text { C } \\
\text { A } \\
\text { P } \\
\text { C }\end{array}$ & \begin{tabular}{|l|l|}
$\mathrm{N}$ & $\mathrm{N}$ \\
$\mathrm{P}$ & $\mathrm{R}$ \\
$\mathrm{R}$ & $\mathrm{R}$ \\
$\mathrm{P}$ & $\mathrm{R}$ \\
$\mathrm{P}$ & $\mathrm{R}$ \\
$\mathrm{P}$ & $\mathrm{R}$ \\
$\mathrm{P}$ & $\mathrm{R}$ \\
\end{tabular} & \begin{tabular}{l|l}
$\mathrm{R}$ & $\mathrm{T}$ \\
$\mathrm{R}$ & \\
$\mathrm{R}$ & \\
$\mathrm{R}$ & $\mathrm{T}$ \\
$\mathrm{R}$ & \\
$\mathrm{R}$ &
\end{tabular} & \begin{tabular}{|l|l|}
$R$ & \\
$R$ & \\
$R$ & \\
$P$ & \\
$R$ & \\
$R$ & \\
\end{tabular} & $\begin{array}{l}\mathrm{P} \\
\mathrm{R} \\
\mathrm{P} \\
\mathrm{P} \\
\mathrm{P} \\
\mathrm{P} \\
\mathrm{T}\end{array}$ & $\begin{array}{l}\mathrm{R} \\
\mathrm{R} \\
\mathrm{R} \\
\mathrm{R} \\
\mathrm{R} \\
\mathrm{R}\end{array}$ & & ${ }_{\mathbf{R}}$ & \begin{tabular}{|l|l}
$\mathrm{T}$ & $\mathrm{R}$ \\
& $\mathrm{R}$ \\
& $\mathrm{T}$ \\
& $\mathrm{R}$ \\
$\mathrm{T}$ & $\mathrm{T}$ \\
$\mathrm{R}$ & $\mathrm{R}$ \\
\end{tabular} & \begin{tabular}{l|l}
$R_{R}$ & $R$ \\
$R$ & \\
$R$ & $T$ \\
$R$ & \\
$R$ &
\end{tabular} & & \\
\hline 42.0 & 5 & \begin{tabular}{l|l|l}
1 & \\
2 & \\
& \\
3 \\
4 \\
5 \\
6
\end{tabular} & $\begin{array}{l}73-74 \\
21-24 \\
77-78 \\
21-26 \\
21-24 \\
21 \cdot 24 \\
21 \cdot 24 \\
21 \cdot 24\end{array}$ & \begin{tabular}{|l|} 
A \\
A \\
F \\
A \\
C \\
F \\
F \\
\end{tabular} & \begin{tabular}{|l|} 
G \\
G \\
G \\
G \\
G \\
G \\
G \\
G
\end{tabular} & \begin{tabular}{|l|}
3 \\
2 \\
2 \\
2 \\
1 \\
1 \\
1 \\
1
\end{tabular} & \begin{tabular}{l|l} 
& \\
$\mathrm{A}$ & \\
$\mathrm{P}$ & \\
$\mathrm{R}$ & \\
$\mathrm{A}$ & \\
$\mathrm{A}$ &
\end{tabular} & \begin{tabular}{l|l}
$\mathrm{C}$ & \\
$\mathrm{C}$ & \\
$\mathrm{A}$ & \\
$\mathrm{R}$ & \\
$\mathrm{C}$ & \\
$\mathrm{A}$ & \\
$\mathrm{R}$ &
\end{tabular} & $\begin{array}{l}\mathrm{C} \\
\mathrm{C} \\
\mathrm{C} \\
\mathrm{R} \\
\mathrm{C} \\
\mathrm{C} \\
\mathrm{R} \\
\mathrm{R}\end{array}$ & \begin{tabular}{|l|l|}
$\mathrm{P}$ & $\mathrm{R}$ \\
$\mathrm{P}$ & $\mathrm{R}$ \\
$\mathrm{C}$ & $\mathrm{R}$ \\
$\mathrm{P}$ & $\mathrm{R}$ \\
$\mathrm{R}$ & $\mathrm{R}$ \\
$\mathrm{R}$ & $\mathrm{R}$ \\
\end{tabular} & \begin{tabular}{l|l}
$R$ & \\
$R$ & $R$ \\
$R$ & $R$ \\
$R$ & \\
$R$ & \\
$R$ & \\
$R$ & \\
\end{tabular} & \begin{tabular}{|l|}
$\mathrm{R}$ \\
$\mathrm{P}$ \\
$\mathrm{P}$ \\
$\mathrm{P}$ \\
$\mathrm{R}$ \\
\end{tabular} & \begin{tabular}{l|}
$\mathrm{P}$ \\
$\mathrm{P}$ \\
$\mathrm{P}$ \\
$\mathrm{C}$ \\
$\mathrm{P}$ \\
$\mathrm{R}$
\end{tabular} & $\begin{array}{l}\mathrm{R} \\
\mathrm{R} \\
\mathrm{P} \\
\mathrm{T} \\
\mathrm{P} \\
\mathrm{R}\end{array}$ & $\begin{array}{l}R \\
R \\
\end{array}$ & \begin{tabular}{l|l}
$\mathrm{R}$ \\
$\mathrm{T}$ \\
\end{tabular} & \begin{tabular}{|l|l}
$\mathrm{R}$ & $\mathrm{R}$ \\
$\mathrm{R}$ & $\mathrm{R}$ \\
$\mathrm{cf}$ & $\mathrm{T}$ \\
$\mathrm{T}$ & $\mathrm{T}$
\end{tabular} & \begin{tabular}{l|l}
$R$ & $R$ \\
$R$ & \\
$R$ & $T$ \\
& $T$
\end{tabular} & & \\
\hline
\end{tabular}

Within the Pliocene sequence there were recognized Sphaeroidinellopsis seminulina, S. subdehiscens, Globorotalia margaritae, Globigerinoides obliquus, Globorotalia acostaensis, G. obesa, Globigerina atlantica, G. apertura, G. cariacoensis, Globorotalia crassula, G. triangula.

Some of these are differentiated in morphotypes; others are grouped together.

\section{FACTORS THAT DISTURB THE BIOSTRATIGRAPHIC RECORD}

The transition of a planktonic foraminiferal biocoenosis to a thanatocoenosis and the preservation of the latter are subject to various factors that are related more or less directly to the submarine topography and the wa- ter depths of the different sites. Hole 548 was near the top of the continental slope that terminates the wide continental shelf (Fig. 1B). It was at $1256 \mathrm{~m}$ below sea level. Hole 549A was at the top of the Pendragon Escarpment, approximately halfway down the continental slope, at a water depth of $2535 \mathrm{~m}$. Hole 550 was on the abyssal plain at $4432 \mathrm{~m}$ water depth.

Bathymetric position has effects with regard to both the reworking of displaced faunas and dissolution.

\section{Reworked and Displaced Faunas}

Reworked and displaced faunas occur in Hole 548 from Core 13 to Core 2, where a benthic microfauna that originated on the continental shelf was observed (Caralp, 
Table 2B. Distribution of planktonic foraminifers in Hole 548 (Cores 6-9).

\begin{tabular}{|c|c|c|c|c|c|c|c|c|c|c|c|c|c|c|c|c|c|c|c|c|c|c|c|c|c|}
\hline 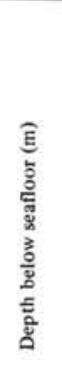 & อั้ & 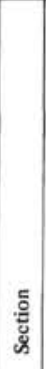 & 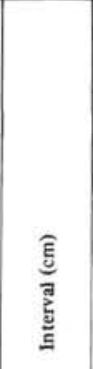 & 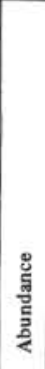 & 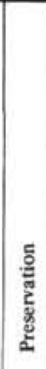 & 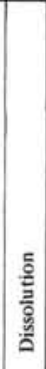 & 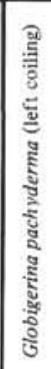 & 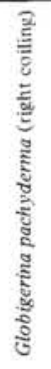 & 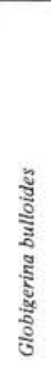 & 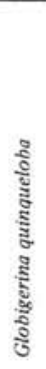 & 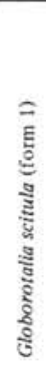 & 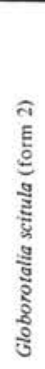 & 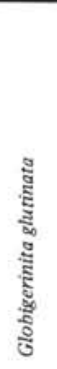 & 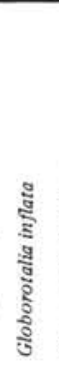 & 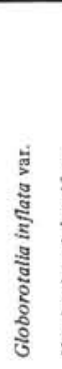 & 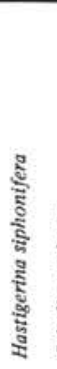 & 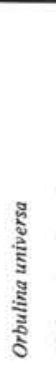 & 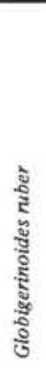 & 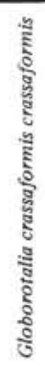 & 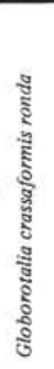 & 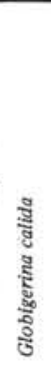 & 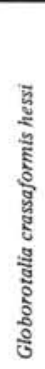 & 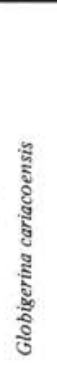 & 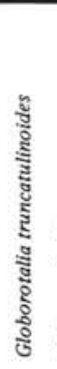 & 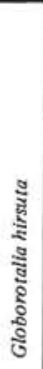 \\
\hline 51.5 & 6 & $\begin{array}{l}1 \\
2 \\
3 \\
4 \\
5 \\
6 \\
7 \\
\end{array}$ & $\begin{array}{l}57-60 \\
16-19 \\
95-98 \\
16-19 \\
16-19 \\
28-29 \\
16-19 \\
13-19 \\
\end{array}$ & $\begin{array}{l}\mathrm{A} \\
\mathrm{A} \\
\mathrm{C} \\
\mathrm{C} \\
\mathrm{C} \\
\mathrm{F} \\
\mathrm{F} \\
\mathrm{F} \\
\end{array}$ & $\begin{array}{l}\mathrm{G} \\
\mathrm{G} \\
\mathrm{M} \\
\mathrm{M} \\
\mathrm{G} \\
\mathrm{G} \\
\mathrm{G} \\
\mathrm{G}\end{array}$ & $\begin{array}{l}3 \\
3 \\
3 \\
2 \\
2 \\
2 \\
1 \\
1 \\
\end{array}$ & \begin{tabular}{|l|}
$\mathrm{R}$ \\
$\mathrm{T}$ \\
$\mathrm{T}$ \\
$\mathrm{A}$ \\
$\mathrm{A}$ \\
$\mathrm{A}$ \\
\end{tabular} & $\begin{array}{l}\mathrm{c} \\
\mathrm{c} \\
\mathrm{c} \\
\mathrm{c} \\
\mathrm{P}\end{array}$ & \begin{tabular}{|l|}
$\mathrm{c}$ \\
$\mathrm{C}$ \\
$\mathrm{P}$ \\
$\mathrm{p}$ \\
$\mathrm{P}$ \\
$\mathrm{R}$
\end{tabular} & $\begin{array}{l}\mathrm{R} \\
\mathrm{P} \\
\mathrm{P} \\
\mathrm{R} \\
\mathrm{R}\end{array}$ & $\begin{array}{l}\mathrm{R} \\
\mathrm{R} \\
\mathrm{R} \\
\mathrm{R} \\
\mathrm{P}\end{array}$ & & \begin{tabular}{|l|}
$\mathbf{P}$ \\
$\mathrm{P}$ \\
$\mathrm{R}$ \\
$\mathrm{R}$
\end{tabular} & $\begin{array}{l}\mathrm{C} \\
\mathrm{C} \\
\mathrm{P} \\
\mathrm{P} \\
\mathrm{C} \\
\mathrm{T}\end{array}$ & & $\begin{array}{l}\mathrm{R} \\
\mathrm{P}\end{array}$ & $\begin{array}{l}\mathrm{C} \\
\mathrm{P} \\
\mathrm{P} \\
\mathrm{T} \\
\mathrm{R}\end{array}$ & $\mathrm{T}$ & & & & & & $\begin{array}{l}\mathrm{R} \\
\mathrm{R} \\
\mathrm{T} \\
\mathrm{R}\end{array}$ & $\mathbf{R}$ \\
\hline 61.0 & 7 & $\begin{array}{l}1 \\
2 \\
3 \\
4 \\
5\end{array}$ & \begin{tabular}{|c|}
$76-79$ \\
$84-85$ \\
$40-43$ \\
$104-111$ \\
$40-43$ \\
$103-110$ \\
$19-22$ \\
$71-72$ \\
$111-116$ \\
$140-141$ \\
$40-43$ \\
$110-117$ \\
$10-13$
\end{tabular} & $\begin{array}{l}\mathrm{F} \\
\mathrm{F} \\
\mathrm{F} \\
\mathrm{F} \\
\mathrm{A} \\
\mathrm{A} \\
\mathrm{A} \\
\mathrm{F} \\
\mathrm{A} \\
\mathrm{A} \\
\mathrm{F} \\
\mathrm{A} \\
\mathrm{C}\end{array}$ & \begin{tabular}{|l} 
G \\
G \\
G \\
G \\
G \\
G \\
G \\
M \\
G \\
G \\
M \\
G \\
M
\end{tabular} & $\begin{array}{l}3 \\
2 \\
3 \\
2 \\
2 \\
2 \\
3 \\
3 \\
2 \\
1 \\
2 \\
2 \\
4\end{array}$ & \begin{tabular}{|l|} 
A \\
A \\
A \\
P \\
\\
\\
C
\end{tabular} & \begin{tabular}{|l|}
$\mathrm{C}$ \\
$\mathrm{R}$ \\
$\mathrm{P}$ \\
$\mathrm{A}$ \\
$\mathrm{C}$ \\
$\mathrm{A}$ \\
$\mathrm{C}$ \\
$\mathrm{A}$ \\
$\mathrm{C}$ \\
$\mathrm{C}$ \\
$\mathrm{A}$ \\
$\mathrm{A}$ \\
$\mathrm{A}$
\end{tabular} & \begin{tabular}{|l|}
$\mathrm{P}$ \\
$\mathrm{R}$ \\
$\mathrm{P}$ \\
$\mathrm{C}$ \\
$\mathrm{P}$ \\
$\mathrm{C}$ \\
$\mathrm{P}$ \\
$\mathrm{P}$ \\
$\mathrm{P}$ \\
$\mathrm{C}$ \\
$\mathrm{C}$ \\
$\mathrm{P}$ \\
$\mathrm{P}$
\end{tabular} & $\begin{array}{l}\mathrm{R} \\
\mathrm{P} \\
\mathrm{R} \\
\mathrm{R} \\
\mathrm{P} \\
\mathrm{P} \\
\mathrm{P} \\
\mathrm{P} \\
\mathrm{R}\end{array}$ & $\begin{array}{l}\mathrm{R} \\
\mathrm{R} \\
\mathrm{R} \\
\mathrm{R} \\
\mathrm{R} \\
\mathrm{R} \\
\mathrm{R} \\
\mathrm{R}\end{array}$ & $\begin{array}{l}\mathrm{R} \\
\mathrm{R} \\
\mathrm{R} \\
\mathrm{R} \\
\mathrm{R} \\
\mathrm{R} \\
\mathrm{R} \\
\mathrm{R} \\
\mathrm{R}\end{array}$ & \begin{tabular}{|l|}
$\mathrm{R}$ \\
$\mathrm{R}$ \\
$\mathrm{R}$ \\
$\mathrm{R}$ \\
$\mathrm{R}$ \\
$\mathrm{P}$ \\
$\mathrm{R}$ \\
$\mathrm{R}$ \\
$\mathrm{A}$ \\
$\mathrm{R}$ \\
$\mathrm{P}$
\end{tabular} & $\begin{array}{l}\mathrm{P} \\
\mathrm{P} \\
\mathrm{C} \\
\mathrm{C} \\
\mathrm{C} \\
\mathrm{P} \\
\mathrm{P} \\
\mathrm{P} \\
\mathrm{P} \\
\mathrm{P} \\
\mathrm{P}\end{array}$ & $\begin{array}{l}\mathrm{R} \\
\mathrm{R}\end{array}$ & $\mathbf{R}$ & $\begin{array}{l}\mathrm{R} \\
\mathrm{R} \\
\mathrm{R} \\
\mathrm{R} \\
\mathrm{R} \\
\mathrm{R} \\
\mathrm{R} \\
\mathrm{R} \\
\mathrm{R} \\
\mathrm{R}\end{array}$ & $\begin{array}{l}\mathbf{R} \\
\mathbf{R}\end{array}$ & $\mathbf{R}$ & & $\begin{array}{l}\mathrm{R} \\
\mathrm{R}\end{array}$ & & $\begin{array}{l}\text { R } \\
\text { P } \\
\text { P }\end{array}$ & $\begin{array}{l}\mathrm{R} \\
\mathrm{R} \\
\mathrm{R} \\
\mathrm{R} \\
\mathrm{R} \\
\mathrm{R}\end{array}$ & $\begin{array}{l}\mathbf{R} \\
\mathrm{R}\end{array}$ \\
\hline 70.5 & 8 & $\begin{array}{l}3 \\
4 \\
5\end{array}$ & $\begin{array}{c}69-70 \\
120-122 \\
40-47 \\
90-91 \\
140-147 \\
69-70 \\
20-27 \\
90-91 \\
20-22 \\
69-70 \\
103-110 \\
69-70 \\
124-125 \\
\end{array}$ & $\begin{array}{l}\mathrm{A} \\
\mathrm{A} \\
\mathrm{A} \\
\mathrm{C} \\
\mathrm{A} \\
\mathrm{C} \\
\mathrm{A} \\
\mathrm{F} \\
\mathrm{A} \\
\mathrm{C} \\
\mathrm{A} \\
\mathrm{A} \\
\mathrm{A}\end{array}$ & \begin{tabular}{|l} 
M \\
G \\
G \\
M \\
G \\
M \\
G \\
M \\
G \\
M \\
G \\
G \\
G \\
\end{tabular} & \begin{tabular}{l|}
3 \\
2 \\
2 \\
2 \\
1 \\
1 \\
1 \\
1 \\
1 \\
2 \\
2 \\
1 \\
1 \\
\end{tabular} & $\begin{array}{l}\mathrm{R} \\
\mathrm{A} \\
\mathrm{A} \\
\mathrm{A}\end{array}$ & \begin{tabular}{l|}
$\mathrm{A}$ \\
$\mathrm{C}$ \\
$\mathrm{C}$ \\
$\mathrm{C}$ \\
$\mathrm{A}$ \\
$\mathrm{A}$ \\
$\mathrm{A}$ \\
$\mathrm{A}$ \\
$\mathrm{A}$ \\
$\mathrm{A}$ \\
$\mathrm{A}$ \\
$\mathrm{A}$ \\
$\mathrm{A}$ \\
\end{tabular} & \begin{tabular}{|l|}
$\mathrm{R}$ \\
$\mathrm{P}$ \\
$\mathrm{C}$ \\
$\mathrm{P}$ \\
$\mathrm{P}$ \\
$\mathrm{P}$ \\
$\mathrm{P}$ \\
$\mathrm{P}$ \\
$\mathrm{C}$ \\
$\mathrm{C}$ \\
$\mathrm{C}$ \\
$\mathrm{C}$ \\
$\mathrm{C}$ \\
\end{tabular} & $\begin{array}{l}\mathrm{R} \\
\mathrm{P} \\
\mathrm{P} \\
\mathrm{R} \\
\mathrm{R} \\
\mathrm{R} \\
\mathrm{R} \\
\mathrm{R}\end{array}$ & \begin{tabular}{|l}
$\mathrm{R}$ \\
$\mathrm{R}$ \\
$\mathrm{R}$ \\
$\mathrm{R}$ \\
$\mathrm{R}$ \\
$\mathrm{R}$ \\
\\
$\mathrm{C}$ \\
$\mathrm{R}$ \\
$\mathrm{R}$ \\
$\mathrm{R}$ \\
\end{tabular} & \begin{tabular}{|l|}
$R$ \\
$R$ \\
$R$ \\
$R$ \\
$R$ \\
\\
$R$ \\
$P$ \\
$R$ \\
$R$ \\
$P$ \\
$R$ \\
$R$ \\
$R$ \\
\end{tabular} & \begin{tabular}{|l|}
$R$ \\
$R$ \\
$P$ \\
$R$ \\
$P$ \\
$R$ \\
$R$ \\
$P$ \\
$R$ \\
$R$ \\
$R$ \\
$R$ \\
$R$ \\
$R$ \\
\end{tabular} & $\begin{array}{l}\mathrm{P} \\
\mathrm{P} \\
\mathrm{P} \\
\mathrm{P} \\
\mathrm{P} \\
\mathrm{P} \\
\mathrm{P} \\
\mathrm{C} \\
\mathrm{C} \\
\mathrm{P} \\
\mathrm{P} \\
\mathrm{P} \\
\end{array}$ & & & $\begin{array}{l}\mathrm{R} \\
\mathrm{R} \\
\mathrm{R} \\
\mathrm{R} \\
\mathrm{R} \\
\mathrm{R} \\
\mathrm{R} \\
\mathrm{R} \\
\mathrm{A} \\
\mathrm{R} \\
\mathrm{R} \\
\mathrm{R} \\
\mathrm{R} \\
\end{array}$ & & $\begin{array}{l}\mathrm{R} \\
\mathrm{R} \\
\mathrm{R} \\
\mathrm{P} \\
\mathrm{R} \\
\mathrm{R} \\
\mathrm{R} \\
\mathrm{T}\end{array}$ & & $\mathbf{R}$ & $\begin{array}{l}\mathrm{R} \\
\mathrm{R} \\
\mathrm{R} \\
\mathrm{P} \\
\mathrm{P} \\
\mathrm{R} \\
\mathrm{T}\end{array}$ & \begin{tabular}{|l|}
$\mathrm{P}$ \\
$\mathrm{P}$ \\
$\mathrm{R}$ \\
$\mathrm{R}$ \\
\\
$\mathrm{R}$ \\
$\mathrm{R}$ \\
$\mathrm{P}$ \\
$\mathrm{T}$ \\
\end{tabular} & $\begin{array}{l}\mathrm{R} \\
\mathrm{R} \\
\mathrm{R} \\
\mathrm{P} \\
\mathrm{R} \\
\\
\mathrm{R} \\
\mathrm{T} \\
\mathrm{T} \\
\mathrm{T}\end{array}$ & \\
\hline 75. & 9 & $\begin{array}{l}1 \\
2\end{array}$ & \begin{tabular}{|c|}
$20-23$ \\
$100-107$ \\
$20-23$ \\
$43-44$ \\
$108-109$ \\
$20-23$
\end{tabular} & $\begin{array}{l}\mathrm{A} \\
\mathrm{A} \\
\mathrm{A} \\
\mathrm{A} \\
\mathrm{A} \\
\mathrm{A}\end{array}$ & $\begin{array}{l}\text { G } \\
\text { G } \\
\text { G } \\
\text { M } \\
\text { G } \\
\text { G }\end{array}$ & $\begin{array}{l}3 \\
1 \\
2 \\
2 \\
1 \\
2\end{array}$ & $\begin{array}{l}\mathrm{R} \\
\mathrm{R} \\
\mathrm{R} \\
\mathrm{R} \\
\mathrm{P}\end{array}$ & \begin{tabular}{l|l|}
$\mathrm{P}$ & \\
$\mathrm{C}$ & $\mathrm{C}$ \\
$\mathrm{C}$ & $\mathrm{c}$ \\
$\mathrm{C}$ & \\
\end{tabular} & \begin{tabular}{|l|}
$\mathrm{c}$ \\
$\mathrm{c}$ \\
$\mathrm{c}$ \\
$\mathrm{c}$ \\
$\mathrm{c}$ \\
$\mathrm{c}$ \\
\end{tabular} & $\begin{array}{l}\mathrm{R} \\
\mathrm{R}\end{array}$ & \begin{tabular}{|l|}
$R$ \\
$R$ \\
$R$ \\
$R$ \\
$P$ \\
$R$
\end{tabular} & $\begin{array}{l}\mathrm{P} \\
\mathrm{R} \\
\\
\mathrm{R} \\
\mathrm{R}\end{array}$ & $\begin{array}{l}\mathrm{P} \\
\mathrm{P} \\
\mathrm{P} \\
\mathrm{P} \\
\mathrm{P}\end{array}$ & $\begin{array}{l}\text { c } \\
\text { c } \\
\text { c } \\
\text { c } \\
\text { c } \\
\text { c }\end{array}$ & & $\begin{array}{l}\mathbf{R} \\
\mathbf{R} \\
\mathbf{R}\end{array}$ & $\begin{array}{l}\mathbf{P} \\
\mathbf{R} \\
\mathbf{R} \\
\mathrm{R}\end{array}$ & $\begin{array}{l}\mathbf{R} \\
\mathrm{R}\end{array}$ & $\begin{array}{l}\mathrm{R} \\
\mathrm{R} \\
\mathrm{R} \\
\mathrm{R}\end{array}$ & \begin{tabular}{|l|}
$\mathbf{R}$ \\
$\mathbf{T}$ \\
$\mathbf{T}$ \\
$\mathbf{T}$
\end{tabular} & $\mathrm{R}$ & \begin{tabular}{|l|}
$\mathrm{R}$ \\
$\mathrm{R}$ \\
$\mathrm{P}$ \\
$\mathrm{P}$
\end{tabular} & $\begin{array}{l}\mathrm{R} \\
\mathrm{T} \\
\mathrm{R} \\
\mathrm{R}\end{array}$ & $\begin{array}{l}R \\
R \\
R \\
R \\
R \\
R\end{array}$ & \\
\hline
\end{tabular}

this volume). Some planktonic microfauna have also been displaced. Redeposition and also grain size sorting by turbidite or contour currents are manifested by the frequent occurrence at some levels of small-sized microfauna and the relative abundance of shell fragments and resistant large forms at other levels. We disregarded samples from these levels in developing the biostratigraphy and reconstructing the paleoclimate.

\section{Dissolution}

In most of the samples examined the microfauna is quite well preserved and shows very few signs of dissolution. Although Hole 550 was at a water depth of 4432 $\mathrm{m}$, dissolution was not particularly pronounced. Nevertheless, several levels, reflecting climatic conditions comparable to the present, show quite distinct evidence of dissolution, with foraminifers slightly affected to damaged. At Holes 549A and 548 the fauna is badly damaged at a few levels also.
The dissolution of planktonic foraminiferal calcitic tests may destroy the faunal association in whole or in part. It changes the nature of the association, which is usually interpreted as reflecting near-surface environmental conditions. An interpretation of environmental conditions must therefore be either disregarded or treated with particular care during biostratigraphic and paleoclimatic analyses. Other factors that can affect environmental reconstruction are drifts and bioturbation, which may mix or disturb original faunal associations. Environmental studies should, to the extent possible, avoid samples affected by any of these factors.

\section{BIOSTRATIGRAPHIC ZONATION}

The biostratigraphy established in this paper for the Quaternary sequences is based on planktonic foraminifers. We used the same faunal elements and criteria for this biostratigraphy as we did elsewhere in the northeast Atlantic (Pujol, 1980). 
Table 2C. Distribution of planktonic foraminifers in Hole 548 (Cores 10-14).

\begin{tabular}{|c|c|c|c|c|c|c|c|c|c|c|c|c|c|c|c|c|c|c|c|c|c|c|c|c|c|}
\hline 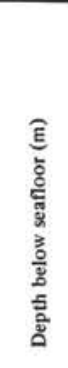 & ठ․ & 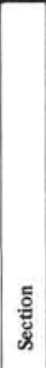 & 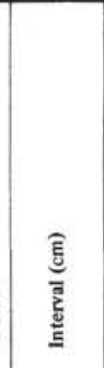 & 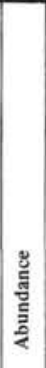 & 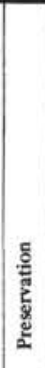 & 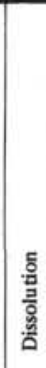 & 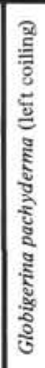 & 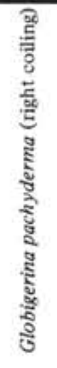 & 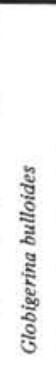 & 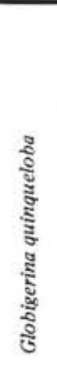 & 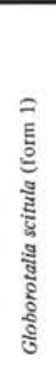 & 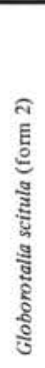 & 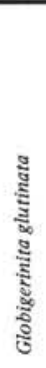 & 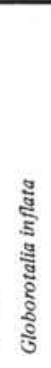 & 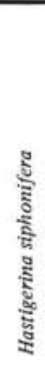 & 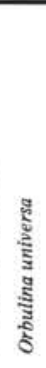 & 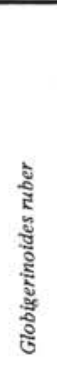 & 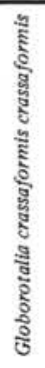 & 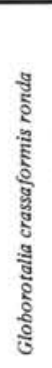 & 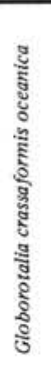 & 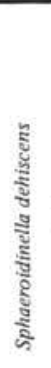 & $\begin{array}{l} \\
\text { ปี } \\
\text { है } \\
\text { : } \\
\text { : } \\
\text { : }\end{array}$ & 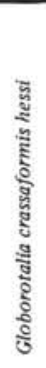 & 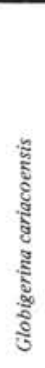 & 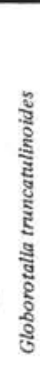 \\
\hline 80.0 & 10 & CC & \begin{tabular}{|c}
$43-44$ \\
$121-122$ \\
$30-31$ \\
$126-129$ \\
$70-71$ \\
$117-711$ \\
$43-44$ \\
$96-99$ \\
$114-116$ \\
$43-44$ \\
$120-122$ \\
$54-58$ \\
$88-90$ \\
\end{tabular} & $\begin{array}{l}\mathrm{A} \\
\mathrm{A} \\
\mathrm{A} \\
\mathrm{F} \\
\mathrm{C} \\
\mathrm{A} \\
\mathrm{A} \\
\mathrm{A} \\
\mathrm{A} \\
\mathrm{A} \\
\mathrm{C} \\
\mathrm{A} \\
\mathrm{C} \\
\mathrm{A}\end{array}$ & $\begin{array}{l}\mathrm{M} \\
\mathrm{M} \\
\mathrm{M} \\
\mathrm{G} \\
\mathrm{G} \\
\mathrm{M} \\
\mathrm{M} \\
\mathrm{M} \\
\mathrm{G} \\
\mathrm{G} \\
\mathrm{M} \\
\mathrm{G} \\
\mathrm{M} \\
\mathrm{G}\end{array}$ & $\begin{array}{l}2 \\
3 \\
1 \\
1 \\
1 \\
2 \\
2 \\
2 \\
3 \\
1 \\
2 \\
1 \\
2 \\
1\end{array}$ & $\begin{array}{l}\mathrm{R} \\
\mathrm{P} \\
\mathrm{C} \\
\mathrm{C} \\
\mathrm{P} \\
\mathrm{A} \\
\mathrm{P} \\
\mathrm{P} \\
\mathrm{P} \\
\mathrm{P} \\
\mathrm{R}\end{array}$ & \begin{tabular}{|l|}
$\mathrm{C}$ \\
$\mathrm{A}$ \\
$\mathrm{A}$ \\
$\mathrm{P}$ \\
$\mathrm{C}$ \\
$\mathrm{A}$ \\
$\mathrm{C}$ \\
$\mathrm{C}$ \\
$\mathrm{A}$ \\
$\mathrm{A}$ \\
$\mathrm{A}$ \\
$\mathrm{A}$ \\
$\mathrm{A}$
\end{tabular} & \begin{tabular}{|l|}
$\mathrm{C}$ \\
$\mathrm{C}$ \\
$\mathrm{P}$ \\
$\mathrm{P}$ \\
$\mathrm{C}$ \\
$\mathrm{C}$ \\
$\mathrm{P}$ \\
$\mathrm{C}$ \\
$\mathrm{C}$ \\
$\mathrm{P}$ \\
$\mathrm{C}$ \\
$\mathrm{C}$ \\
$\mathrm{A}$
\end{tabular} & \begin{tabular}{|l|}
$\mathrm{R}$ \\
$\mathrm{R}$ \\
$\mathrm{R}$ \\
$\mathrm{P}$ \\
\\
$\mathrm{R}$ \\
$\mathrm{R}$ \\
$\mathrm{P}$ \\
$\mathrm{R}$ \\
$\mathrm{R}$ \\
$\mathrm{R}$ \\
$\mathrm{R}$ \\
$\mathrm{P}$
\end{tabular} & \begin{tabular}{|l} 
R \\
T \\
T \\
\\
R \\
T \\
T \\
\\
T
\end{tabular} & $\begin{array}{l}\mathrm{R} \\
\mathrm{R} \\
\mathrm{R} \\
\mathrm{R}\end{array}$ & \begin{tabular}{|l}
$\mathrm{P}$ \\
$\mathrm{P}$ \\
$\mathrm{R}$ \\
$\mathrm{P}$ \\
$\mathrm{R}$ \\
$\mathrm{P}$ \\
$\mathrm{R}$ \\
$\mathrm{R}$ \\
$\mathrm{P}$ \\
$\mathrm{R}$ \\
$\mathrm{R}$ \\
$\mathrm{R}$ \\
$\mathrm{R}$ \\
$\mathrm{R}$
\end{tabular} & $\begin{array}{l}\mathrm{P} \\
\mathrm{C} \\
\mathrm{C} \\
\mathrm{P} \\
\mathrm{R} \\
\mathrm{C} \\
\mathrm{C} \\
\mathrm{R} \\
\mathrm{P} \\
\mathrm{P} \\
\mathrm{P} \\
\mathrm{P} \\
\mathrm{P} \\
\mathrm{C}\end{array}$ & $\begin{array}{l}\mathrm{P} \\
\mathrm{R} \\
\mathrm{R} \\
\mathrm{R} \\
\mathrm{R} \\
\mathrm{R} \\
\mathrm{R} \\
\mathrm{P}\end{array}$ & \begin{tabular}{|l}
$\mathrm{R}$ \\
$\mathrm{R}$ \\
$\mathrm{P}$ \\
$\mathrm{P}$ \\
$\mathrm{R}$ \\
$\mathrm{R}$ \\
$\mathrm{R}$ \\
$\mathrm{R}$ \\
$\mathrm{R}$
\end{tabular} & \begin{tabular}{|l}
$\mathrm{R}$ \\
$\mathrm{R}$ \\
$\mathrm{R}$ \\
$\mathrm{R}$ \\
$\mathrm{R}$
\end{tabular} & \begin{tabular}{|l}
$\mathrm{R}$ \\
$\mathrm{R}$ \\
$\mathrm{R}$
\end{tabular} & \begin{tabular}{|l}
$\mathrm{R}$ \\
$\mathrm{R}$ \\
$\mathrm{R}$
\end{tabular} & $\mathbf{R}$ & & $\begin{array}{l}\mathrm{R} \\
\mathrm{R}\end{array}$ & $\mathrm{T}$ & R & \begin{tabular}{|l}
$\mathrm{R}$ \\
$\mathrm{T}$ \\
$\mathrm{R}$ \\
$\mathrm{T}$ \\
$\mathrm{P}$
\end{tabular} \\
\hline 82.5 & 11 & $\begin{array}{r}6 \\
\text { CC }\end{array}$ & \begin{tabular}{|c|}
$52-53$ \\
$73-74$ \\
$140-147$ \\
$50-53$ \\
$140-147$ \\
$50-53$ \\
$139-146$ \\
$50-53$ \\
$140-147$ \\
$17-24$ \\
$50-53$ \\
$92-97$ \\
$143-150$ \\
$8-15$ \\
\end{tabular} & \begin{tabular}{|l|}
$\mathrm{A}$ \\
$\mathrm{C}$ \\
$\mathrm{A}$ \\
$\mathrm{F}$ \\
$\mathrm{A}$ \\
$\mathrm{C}$ \\
$\mathrm{A}$ \\
$\mathrm{C}$ \\
$\mathrm{C}$ \\
$\mathrm{A}$ \\
$\mathrm{A}$ \\
$\mathrm{A}$ \\
$\mathrm{A}$ \\
$\mathrm{A}$ \\
$\mathrm{A}$ \\
\end{tabular} & $\begin{array}{l}\text { G } \\
\text { M } \\
\text { G } \\
\text { M } \\
\text { G } \\
\text { G } \\
\text { G } \\
\text { M } \\
\text { G } \\
\text { G } \\
\text { G } \\
\text { G } \\
\text { G } \\
\text { G } \\
\text { G }\end{array}$ & $\begin{array}{l}1 \\
2 \\
1 \\
3 \\
1 \\
3 \\
1 \\
2 \\
1 \\
1 \\
1 \\
1 \\
1 \\
2 \\
1 \\
\end{array}$ & ${ }_{R}^{R}$ & $\begin{array}{l}\mathrm{A} \\
\mathrm{A} \\
\mathrm{A} \\
\mathrm{A} \\
\mathrm{A} \\
\mathrm{A} \\
\mathrm{A} \\
\mathrm{A} \\
\mathrm{A} \\
\mathrm{A} \\
\mathrm{A} \\
\mathrm{C} \\
\mathrm{C} \\
\mathrm{A} \\
\mathrm{A}\end{array}$ & \begin{tabular}{|l|}
$\mathrm{C}$ \\
$\mathrm{A}$ \\
$\mathrm{R}$ \\
$\mathrm{C}$ \\
$\mathrm{R}$ \\
$\mathrm{C}$ \\
$\mathrm{R}$ \\
$\mathrm{C}$ \\
$\mathrm{C}$ \\
$\mathrm{A}$ \\
$\mathrm{A}$ \\
$\mathrm{A}$ \\
$\mathrm{A}$ \\
$\mathrm{A}$
\end{tabular} & $\begin{array}{l}\mathrm{R} \\
\mathrm{R} \\
\mathrm{R} \\
\mathrm{R} \\
\mathrm{R} \\
\mathrm{R} \\
\mathrm{P} \\
\mathrm{P}\end{array}$ & $\begin{array}{l}\mathrm{R} \\
\mathrm{R}\end{array}$ & $\begin{array}{l}\mathrm{R} \\
\mathrm{R} \\
\mathrm{R} \\
\mathrm{R} \\
\mathrm{R}\end{array}$ & $\begin{array}{ll}\mathrm{R} \\
\mathrm{R} \\
\mathrm{P} \\
\mathrm{P} \\
\mathrm{R} \\
\mathrm{R} \\
\mathrm{R} \\
\mathrm{R} \\
\mathrm{R} \\
\mathrm{R} \\
\mathrm{R} \\
\mathrm{R} \\
\mathrm{R} \\
\mathrm{R} \\
\mathrm{R}\end{array}$ & $\begin{array}{l}\mathrm{P} \\
\mathrm{P} \\
\mathrm{P} \\
\mathrm{P} \\
\mathrm{P} \\
\mathrm{P} \\
\mathrm{P} \\
\mathrm{R} \\
\mathrm{C} \\
\mathrm{C} \\
\mathrm{C} \\
\mathrm{C} \\
\mathrm{C} \\
\mathrm{C}\end{array}$ & $\begin{array}{l}\mathrm{R} \\
\mathrm{R} \\
\mathrm{R} \\
\mathrm{P} \\
\mathrm{R} \\
\mathrm{R} \\
\mathrm{T} \\
\mathrm{R} \\
\mathrm{R} \\
\mathrm{R} \\
\mathrm{R} \\
\mathrm{P}\end{array}$ & \begin{tabular}{|l}
$\mathrm{R}$ \\
$\mathrm{R}$ \\
$\mathrm{R}$ \\
$\mathrm{P}$ \\
$\mathrm{R}$ \\
$\mathrm{R}$ \\
$\mathrm{T}$ \\
$\mathrm{R}$ \\
$\mathrm{R}$ \\
$\mathrm{R}$ \\
$\mathrm{P}$ \\
$\mathrm{P}$ \\
$\mathrm{R}$ \\
$\mathrm{P}$
\end{tabular} & $\begin{array}{l}\mathrm{R} \\
\mathrm{R} \\
\mathrm{R} \\
\mathrm{R} \\
\mathrm{R} \\
\\
\mathrm{R} \\
\mathrm{R} \\
\mathrm{P} \\
\mathrm{R} \\
\mathrm{R} \\
\end{array}$ & \begin{tabular}{|l|}
$\mathrm{P}$ \\
$\mathrm{R}$ \\
$\mathrm{P}$ \\
$\mathrm{R}$ \\
$\mathrm{T}$ \\
$\mathrm{R}$ \\
$\mathrm{T}$ \\
$\mathrm{R}$ \\
$\mathrm{R}$ \\
$\mathrm{R}$ \\
$\mathrm{R}$ \\
\end{tabular} & $\begin{array}{l}\mathrm{P} \\
\mathrm{P} \\
\mathrm{R} \\
\mathrm{R} \\
\mathrm{R} \\
\mathrm{T} \\
\mathrm{R} \\
\mathrm{R} \\
\mathrm{R} \\
\mathrm{R} \\
\mathrm{R}\end{array}$ & P & R & $\begin{array}{l}\mathrm{R} \\
\mathrm{R}\end{array}$ & $\begin{array}{l}\mathrm{R} \\
\mathrm{R} \\
\mathrm{R} \\
\mathrm{T} \\
\mathrm{R}\end{array}$ & & \begin{tabular}{|l}
$\mathrm{R}$ \\
$\mathrm{R}$ \\
$\mathrm{R}$ \\
$\mathrm{T}$
\end{tabular} \\
\hline 82.3 & 12 & 1 & \begin{tabular}{|c|}
$129-130$ \\
$36 \cdot 37$
\end{tabular} & \begin{tabular}{|l}
$A$ \\
$A$ \\
$F$ \\
$A$
\end{tabular} & \begin{tabular}{|l} 
G \\
G \\
G
\end{tabular} & \begin{tabular}{|l}
1 \\
2 \\
3 \\
2
\end{tabular} & \begin{tabular}{|l|}
$\mathrm{A}$ \\
$\mathrm{A}$ \\
$\mathrm{C}$ \\
$\mathrm{P}$
\end{tabular} & \begin{tabular}{|l} 
A \\
C \\
A
\end{tabular} & \begin{tabular}{|l|}
$\mathrm{C}$ \\
$\mathrm{C}$ \\
$\mathrm{A}$ \\
\end{tabular} & \begin{tabular}{|l}
$R$ \\
$R$ \\
$P$
\end{tabular} & $\begin{array}{l}\mathrm{R} \\
\mathrm{R} \\
\mathrm{R}\end{array}$ & \begin{tabular}{|l} 
\\
$R$
\end{tabular} & \begin{tabular}{|l}
$\mathrm{R}$ \\
$\mathrm{R}$ \\
$\mathrm{R}$
\end{tabular} & $\begin{array}{l}\text { c } \\
\text { c } \\
\text { c }\end{array}$ & $\mathrm{R}$ & \begin{tabular}{|l}
$\mathrm{R}$ \\
$\mathrm{R}$ \\
\end{tabular} & $\mathrm{R}$ & & & & & & & & R \\
\hline 99.0 & 13 & $\begin{array}{l}1 \\
2\end{array}$ & \begin{tabular}{|c|}
$130-137$ \\
$35-38$ \\
$120-121$ \\
$137-138$ \\
$22-29$ \\
$105-106$ \\
$120-121$ \\
$130-131$ \\
$35-38$ \\
$95-96$ \\
$35-38$ \\
$130-132$ \\
$27-34$ \\
$81-82$ \\
\end{tabular} & \begin{tabular}{|l|}
$\mathrm{A}$ \\
$\mathrm{C}$ \\
$\mathrm{A}$ \\
$\mathrm{A}$ \\
$\mathrm{A}$ \\
$\mathrm{C}$ \\
$\mathrm{A}$ \\
$\mathrm{A}$ \\
$\mathrm{A}$ \\
$\mathrm{A}$ \\
$\mathrm{A}$ \\
$\mathrm{A}$ \\
$\mathrm{A}$ \\
$\mathrm{A}$ \\
$\mathrm{A}$ \\
\end{tabular} & \begin{tabular}{|l}
$G$ \\
$G$ \\
$G$ \\
$M$ \\
G \\
M \\
$G$ \\
$G$ \\
$G$ \\
$G$ \\
$G$ \\
$G$ \\
$G$ \\
$G$ \\
$G$ \\
$G$ \\
\end{tabular} & $\begin{array}{l}1 \\
2 \\
3 \\
3 \\
2 \\
3 \\
2 \\
2 \\
3 \\
3 \\
2 \\
2 \\
2 \\
2 \\
2 \\
\end{array}$ & \begin{tabular}{|l}
$\mathrm{R}$ \\
$\mathrm{P}$ \\
$\mathrm{A}$ \\
$\mathrm{C}$ \\
$\mathrm{C}$ \\
$\mathrm{C}$ \\
$\mathrm{C}$ \\
$\mathrm{A}$ \\
$\mathrm{C}$ \\
$\mathrm{C}$ \\
$\mathrm{C}$ \\
$\mathrm{C}$ \\
$\mathrm{P}$ \\
$\mathrm{R}$
\end{tabular} & $\begin{array}{l}\text { A } \\
\text { C } \\
\text { A } \\
\text { C } \\
\text { C } \\
\text { C } \\
\text { C } \\
\text { C } \\
\text { A } \\
\text { C } \\
\text { C } \\
\text { C } \\
\text { C } \\
\text { C } \\
\text { C }\end{array}$ & \begin{tabular}{|l|}
$\mathrm{C}$ \\
$\mathrm{P}$ \\
$\mathrm{P}$ \\
$\mathrm{P}$ \\
$\mathrm{A}$ \\
$\mathrm{P}$ \\
$\mathrm{P}$ \\
$\mathrm{P}$ \\
$\mathrm{P}$ \\
$\mathrm{C}$ \\
$\mathrm{C}$ \\
$\mathrm{A}$ \\
$\mathrm{C}$ \\
$\mathrm{C}$ \\
$\mathrm{C}$ \\
\end{tabular} & R & $\begin{array}{l}R \\
R \\
R \\
R \\
R \\
R \\
R \\
R \\
R \\
R\end{array}$ & \begin{tabular}{|l|}
$\mathrm{R}$ \\
$\mathrm{R}$ \\
$\mathrm{R}$ \\
$\mathrm{R}$ \\
$\mathrm{R}$ \\
$\mathrm{R}$ \\
$\mathrm{R}$ \\
$\mathrm{R}$ \\
\end{tabular} & $\begin{array}{l}R \\
R \\
R \\
R \\
R \\
R \\
T \\
R \\
R \\
R \\
R \\
R \\
R \\
R \\
R \\
R \\
R \\
R\end{array}$ & $\begin{array}{l}\mathrm{C} \\
\mathrm{C} \\
\mathrm{p} \\
\mathrm{p} \\
\mathrm{c} \\
\mathrm{p} \\
\mathrm{P} \\
\mathrm{P} \\
\mathrm{C} \\
\mathrm{C} \\
\mathrm{c} \\
\mathrm{c} \\
\mathrm{c} \\
\mathrm{c} \\
\mathrm{C}\end{array}$ & \begin{tabular}{|l}
$\mathrm{R}$ \\
$\mathrm{R}$ \\
$\mathrm{R}$ \\
$\mathrm{R}$ \\
$\mathrm{R}$ \\
$\mathrm{R}$ \\
$\mathrm{R}$ \\
$\mathrm{R}$ \\
$\mathrm{R}$ \\
$\mathrm{R}$ \\
$\mathrm{P}$
\end{tabular} & $\begin{array}{l}\mathrm{I} \\
\mathrm{P} \\
\mathrm{R} \\
\mathrm{R} \\
\mathrm{P} \\
\mathrm{R} \\
\mathrm{R} \\
\mathrm{R} \\
\mathrm{R} \\
\mathrm{R} \\
\mathrm{R} \\
\mathrm{P} \\
\mathrm{R} \\
\mathrm{R} \\
\mathrm{P}\end{array}$ & \begin{tabular}{|l|}
$\mathbf{R}$ \\
$\mathrm{T}$
\end{tabular} & \begin{tabular}{|l}
$\mathrm{T}$ \\
$\mathrm{R}$ \\
$\mathrm{T}$ \\
$\mathrm{R}$ \\
$\mathrm{R}$ \\
$\mathrm{P}$
\end{tabular} & T & & & \begin{tabular}{|l} 
R \\
R \\
R \\
R \\
R \\
R \\
R \\
\\
R \\
R \\
R \\
R \\
R \\
\end{tabular} & \begin{tabular}{|l|}
$\mathbf{R}$ \\
$\mathrm{R}$
\end{tabular} & & \\
\hline 100.5 & 14 & Cc & $\begin{array}{r}4-7 \\
40-47 \\
84-91\end{array}$ & $\begin{array}{l}\text { A } \\
\text { A } \\
\text { A } \\
\text { A }\end{array}$ & \begin{tabular}{|l} 
M \\
G \\
G \\
G
\end{tabular} & $\begin{array}{l}3 \\
2 \\
1 \\
2\end{array}$ & R & \begin{tabular}{|l|}
$\mathrm{R}$ \\
$\mathrm{C}$ \\
$\mathrm{C}$ \\
$\mathrm{A}$
\end{tabular} & \begin{tabular}{|l} 
C \\
C \\
A \\
A
\end{tabular} & & $\begin{array}{l}\mathrm{T} \\
\mathrm{R}\end{array}$ & \begin{tabular}{|l|}
$\mathrm{R}$ \\
$\mathrm{R}$ \\
$\mathrm{R}$ \\
$\mathrm{P}$ \\
\end{tabular} & \begin{tabular}{|l|}
$\mathbf{R}$ \\
$\mathrm{R}$ \\
$\mathrm{R}$ \\
$\mathrm{R}$ \\
\end{tabular} & \begin{tabular}{|l|} 
c \\
c \\
c \\
c
\end{tabular} & \begin{tabular}{|l|}
$\mathrm{R}$ \\
$\mathrm{P}$ \\
$\mathrm{P}$ \\
$\mathrm{P}$
\end{tabular} & \begin{tabular}{|l|}
$\mathrm{R}$ \\
$\mathrm{R}$ \\
$\mathrm{P}$ \\
$\mathrm{P}$
\end{tabular} & $\begin{array}{l}\mathrm{R} \\
\mathrm{R}\end{array}$ & \begin{tabular}{|l|}
$R$ \\
$P$ \\
$P$
\end{tabular} & $\begin{array}{l}\mathrm{R} \\
\mathrm{R}\end{array}$ & & & $\begin{array}{l}\mathrm{R} \\
\mathrm{R} \\
\mathrm{R} \\
\mathrm{R}\end{array}$ & & & \\
\hline
\end{tabular}

The Quaternary sequences at this midlatitude location in the North Atlantic have been given their character by the median position of the sites between the present and maximum $\left(42^{\circ} \mathrm{N}\right)$ extent of glaciation (CLIMAP [Climate Long Range Investigation Mapping and Prediction] Project Members, 1976; Ruddiman and McIntyre, 1976). The biozonation must, therefore, take into account climatic fluctuations, which may be used to define bioclimatic subdivisions. These subdivisions are integrated with a biozonation that is defined by the following elements: species appearances and disappear- ances, the frequency and coiling ratio of Globigerina pachyderma, the presence and coiling ratio of Globorotalia hirsuta and $G$. truncatulinoides, and the presence of subtropical and tropical species ( $G$. crassaformis, Globigerinoides ruber, G. conglobatus, and Globorotalia menardii).

Four zones, two of which were divided into three subzones each, were distinguished within the sedimentary Pliocene-Quaternary sequence at Holes 548, 549A, and 550. Figure 2 shows these zones and the species events used to define them. The base of the Quaternary was placed at the 
Table 2D. Distribution of planktonic foraminifers in Hole 548 (Cores 15-18).

\begin{tabular}{|c|c|c|c|c|c|c|c|c|c|c|c|c|c|c|c|c|c|c|c|c|c|c|c|c|c|c|c|c|c|}
\hline 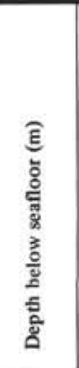 & ชั & 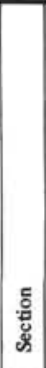 & 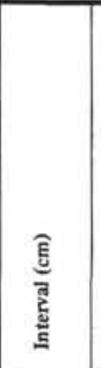 & 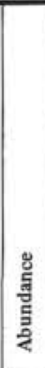 & 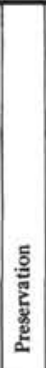 & 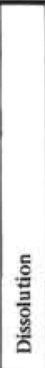 & 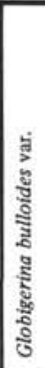 & 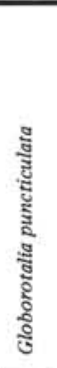 & 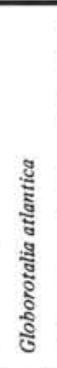 & 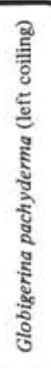 & 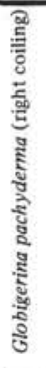 & 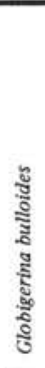 & 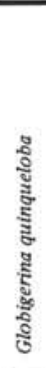 & 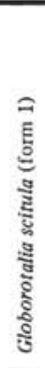 & 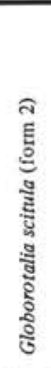 & 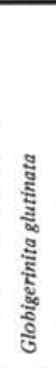 & 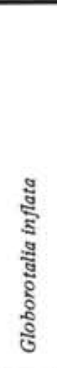 & 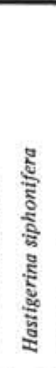 & 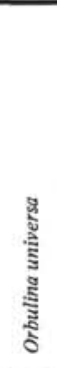 & 气ूँ & 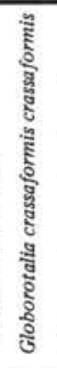 & 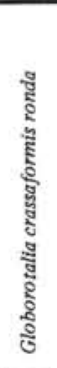 & 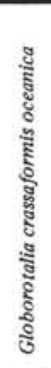 & 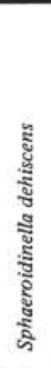 & 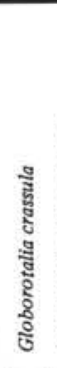 & 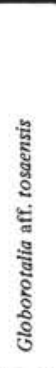 & $\begin{array}{l}\text { : } \\
\text { जूँ } \\
\text { है } \\
\text { हैँ } \\
\text { जँ }\end{array}$ & 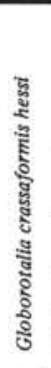 & 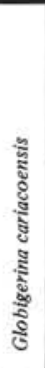 \\
\hline 108.5 & 15 & $\begin{array}{l}1 \\
2 \\
3\end{array}$ & \begin{tabular}{|c|}
$44-45$ \\
$112-119$ \\
$30-33$ \\
$112-119$ \\
$30-33$ \\
$110-117$ \\
$3-4$ \\
$12-13$ \\
$27-28$ \\
$110-117$ \\
$30-33$ \\
$63-68$ \\
$92-97$ \\
$130-137$ \\
$30-33$ \\
$120-123$
\end{tabular} & \begin{tabular}{|l}
$\mathrm{C}$ \\
$\mathrm{A}$ \\
$\mathrm{A}$ \\
$\mathrm{A}$ \\
$\mathrm{A}$ \\
$\mathrm{A}$ \\
$\mathrm{C}$ \\
$\mathrm{A}$ \\
$\mathrm{C}$ \\
$\mathrm{A}$ \\
$\mathrm{A}$ \\
$\mathrm{A}$ \\
$\mathrm{A}$ \\
$\mathrm{A}$ \\
$\mathrm{A}$ \\
$\mathrm{C}$ \\
$\mathrm{A}$
\end{tabular} & $\begin{array}{l}\mathrm{M} \\
\mathrm{G} \\
\mathrm{G} \\
\mathrm{G} \\
\mathrm{G} \\
\mathrm{G} \\
\mathrm{M} \\
\mathrm{G} \\
\mathrm{M} \\
\mathrm{G} \\
\mathrm{M} \\
\mathrm{G} \\
\mathrm{G} \\
\mathrm{G} \\
\mathrm{G} \\
\mathrm{G} \\
\mathrm{G}\end{array}$ & $\begin{array}{l}3 \\
1 \\
2 \\
1 \\
3 \\
1 \\
3 \\
1 \\
3 \\
1 \\
2 \\
1 \\
1 \\
1 \\
2 \\
1 \\
1\end{array}$ & & & & $\begin{array}{l}\mathrm{P} \\
\mathrm{P} \\
\mathrm{C} \\
\mathrm{P} \\
\mathrm{P} \\
\mathrm{P} \\
\mathrm{P} \\
\mathrm{R}\end{array}$ & $\begin{array}{l}\mathrm{C} \\
\mathrm{C} \\
\mathrm{A} \\
\mathrm{A} \\
\mathrm{C} \\
\mathrm{A} \\
\mathrm{C} \\
\mathrm{A} \\
\mathrm{A} \\
\mathrm{A} \\
\mathrm{A} \\
\mathrm{A} \\
\mathrm{A} \\
\mathrm{A} \\
\mathrm{A} \\
\mathrm{A} \\
\mathrm{A}\end{array}$ & $\begin{array}{l}\mathrm{c} \\
\mathrm{C} \\
\mathrm{C} \\
\mathrm{C} \\
\mathrm{C} \\
\mathrm{C} \\
\mathrm{C} \\
\mathrm{c} \\
\mathrm{C} \\
\mathrm{C} \\
\mathrm{c} \\
\mathrm{C} \\
\mathrm{C} \\
\mathrm{C} \\
\mathrm{C} \\
\mathrm{P} \\
\mathrm{C}\end{array}$ & \begin{tabular}{|l}
$\mathrm{R}$ \\
$\mathrm{R}$ \\
$\mathrm{R}$ \\
$\mathrm{R}$ \\
$\mathrm{R}$
\end{tabular} & $\begin{array}{l}\mathrm{R} \\
\mathrm{R} \\
\mathrm{R} \\
\mathrm{R} \\
\mathrm{R} \\
\mathrm{R} \\
\mathrm{P} \\
\mathrm{P} \\
\mathrm{P} \\
\mathrm{P} \\
\mathrm{R} \\
\mathrm{R} \\
\mathrm{R} \\
\mathrm{T} \\
\mathrm{R}\end{array}$ & \begin{tabular}{|l}
$\mathrm{R}$ \\
$\mathrm{R}$ \\
$\mathrm{R}$ \\
$\mathrm{R}$ \\
$\mathrm{R}$ \\
$\mathrm{R}$ \\
$\mathrm{R}$ \\
$\mathrm{R}$ \\
$\mathrm{P}$ \\
$\mathrm{R}$ \\
$\mathrm{P}$ \\
$\mathrm{P}$ \\
$\mathrm{P}$ \\
$\mathrm{P}$ \\
$\mathrm{P}$ \\
$\mathrm{P}$
\end{tabular} & \begin{tabular}{|l}
$R$ \\
$P$ \\
$R$ \\
$R$ \\
$R$ \\
$R$ \\
$R$ \\
$R$ \\
$R$ \\
$R$ \\
$R$ \\
$R$ \\
$R$ \\
$R$ \\
$R$ \\
$R$ \\
$R$ \\
$R$
\end{tabular} & $\begin{array}{l}\mathrm{C} \\
\mathrm{C} \\
\mathrm{C} \\
\mathrm{C} \\
\mathrm{C} \\
\mathrm{C} \\
\mathrm{C} \\
\mathrm{C} \\
\mathrm{C} \\
\mathrm{C} \\
\mathrm{C} \\
\mathrm{C} \\
\mathrm{C} \\
\mathrm{C} \\
\mathrm{C} \\
\mathrm{R} \\
\mathrm{R}\end{array}$ & \begin{tabular}{|l}
$\mathrm{P}$ \\
$\mathrm{P}$ \\
$\mathrm{R}$ \\
$\mathrm{R}$ \\
$\mathrm{R}$ \\
\\
\\
$\mathrm{R}$ \\
$\mathrm{R}$ \\
$\mathrm{R}$ \\
$\mathrm{R}$ \\
$\mathrm{R}$
\end{tabular} & $\begin{array}{l}\mathrm{R} \\
\mathrm{P} \\
\mathrm{R} \\
\mathrm{P} \\
\mathrm{R} \\
\mathrm{R} \\
\mathrm{R} \\
\mathrm{R} \\
\mathrm{R} \\
\mathrm{R} \\
\mathrm{R} \\
\mathrm{R} \\
\mathrm{R} \\
\mathrm{R} \\
\mathrm{R} \\
\mathrm{R} \\
\end{array}$ & $\mathbf{R}$ & $\begin{array}{l}\mathrm{P} \\
\mathrm{P} \\
\mathrm{R} \\
\mathrm{R}\end{array}$ & $\begin{array}{l}\mathrm{R} \\
\mathrm{P}\end{array}$ & $\begin{array}{l}\mathrm{T} \\
\mathrm{R}\end{array}$ & $\mathbf{T}$ & R & $\mathrm{T}$ & $\mathbf{R}$ & ef & $\mathrm{T}$ \\
\hline 118.0 & 16 & $\begin{array}{l}3 \\
4\end{array}$ & \begin{tabular}{|c|}
$10-11$ \\
$70-77$ \\
$140-141$ \\
$20-23$ \\
$70-77$ \\
$140-141$ \\
$50-57$ \\
$140-141$ \\
$10-11$ \\
$70-77$ \\
$140-141$ \\
$10-11$ \\
$70-77$ \\
$140-141$ \\
\end{tabular} & $\begin{array}{l}\mathrm{C} \\
\mathrm{A} \\
\mathrm{F} \\
\mathrm{F} \\
\mathrm{A} \\
\mathrm{A} \\
\mathrm{F} \\
\mathrm{F} \\
\mathrm{R} \\
\mathrm{A} \\
\mathrm{F} \\
\mathrm{F} \\
\mathrm{A} \\
\mathrm{A} \\
\mathrm{A}\end{array}$ & \begin{tabular}{|l}
$\mathrm{M}$ \\
$\mathrm{G}$ \\
$\mathrm{G}$ \\
$\mathrm{M}$ \\
$\mathrm{G}$ \\
$\mathrm{G}$ \\
$\mathrm{G}$ \\
$\mathrm{G}$ \\
$\mathrm{M}$ \\
$\mathrm{G}$ \\
$\mathrm{M}$ \\
$\mathrm{G}$ \\
$\mathrm{G}$ \\
$\mathrm{G}$ \\
$\mathrm{M}$
\end{tabular} & $\begin{array}{l}2 \\
2 \\
2 \\
2 \\
2 \\
2 \\
1 \\
2 \\
\\
3 \\
3 \\
2 \\
2 \\
3 \\
3\end{array}$ & & & $\mathbf{R}$ & & $\begin{array}{l}\mathrm{A} \\
\mathrm{A} \\
\mathrm{A} \\
\mathrm{A} \\
\mathrm{A} \\
\mathrm{A} \\
\mathrm{A} \\
\mathrm{A} \\
\mathrm{A} \\
\mathrm{A} \\
\mathrm{A} \\
\mathrm{A} \\
\mathrm{A} \\
\mathrm{C} \\
\mathrm{A}\end{array}$ & $\begin{array}{l}\mathrm{C} \\
\mathrm{P} \\
\mathrm{C} \\
\mathrm{R} \\
\mathrm{P} \\
\mathrm{P} \\
\mathrm{P} \\
\mathrm{C} \\
\mathrm{A} \\
\mathrm{C} \\
\mathrm{R} \\
\mathrm{P} \\
\mathrm{C} \\
\mathrm{C} \\
\mathrm{A}\end{array}$ & & \begin{tabular}{|l}
$\mathrm{R}$ \\
$\mathrm{T}$ \\
$\mathrm{T}$ \\
$\mathrm{R}$ \\
$\mathrm{R}$ \\
$\mathrm{R}$
\end{tabular} & $\begin{array}{l}\mathrm{T} \\
\mathrm{P} \\
\mathrm{T} \\
\mathrm{R} \\
\mathrm{R} \\
\mathrm{R}\end{array}$ & $\begin{array}{l}\mathrm{R} \\
\mathrm{R} \\
\mathrm{R}\end{array}$ & $\mathbf{R}$ & $\begin{array}{l}\mathrm{T} \\
\mathrm{R} \\
\mathrm{R} \\
\mathrm{T} \\
\mathrm{T}\end{array}$ & \begin{tabular}{|l|}
$R$ \\
$R$ \\
$T$ \\
\\
$R$ \\
$R$ \\
$P$ \\
\\
\\
$P$ \\
$T$ \\
$R$ \\
$P$ \\
$R$ \\
$R$ \\
\end{tabular} & $\mathrm{~T}$ & $\begin{array}{l}R \\
R \\
T \\
R \\
R \\
R\end{array}$ & $\begin{array}{l}\mathbf{C} \\
\mathbf{C} \\
\mathbf{T}\end{array}$ & $\begin{array}{l}\mathrm{R} \\
\mathrm{T}\end{array}$ & $\mathbf{T}$ & & & & & \\
\hline 126.0 & \begin{tabular}{|l|}
17 \\
\end{tabular} & $\begin{array}{l}1 \\
2\end{array}$ & \begin{tabular}{|c|}
$53-57$ \\
$113-120$ \\
$50-57$ \\
$105-112$ \\
$51-58$ \\
$103-110$ \\
$53-56$ \\
$100-107$ \\
$50-57$ \\
\end{tabular} & $\begin{array}{l}\mathrm{C} \\
\mathrm{A} \\
\mathrm{A} \\
\mathrm{C} \\
\mathrm{A} \\
\mathrm{A} \\
\mathrm{F} \\
\mathrm{A} \\
\mathrm{A} \\
\mathrm{A}\end{array}$ & $\begin{array}{l}\text { G } \\
\text { G } \\
\text { G } \\
\text { M } \\
\text { G } \\
\text { G } \\
\text { G } \\
\text { G } \\
\text { G } \\
\text { G }\end{array}$ & \begin{tabular}{|l}
2 \\
2 \\
3 \\
2 \\
2 \\
1 \\
3 \\
1 \\
2 \\
1
\end{tabular} & & & $\begin{array}{l}\mathrm{A} \\
\mathrm{P} \\
\mathrm{P} \\
\mathrm{P} \\
\mathrm{A} \\
\mathrm{C} \\
\mathrm{C} \\
\mathrm{P} \\
\mathrm{C} \\
\mathrm{C}\end{array}$ & R & \begin{tabular}{|l|}
$\mathrm{C}$ \\
$\mathrm{A}$ \\
$\mathrm{R}$ \\
$\mathrm{R}$ \\
$\mathrm{C}$ \\
$\mathrm{P}$ \\
$\mathrm{C}$ \\
$\mathrm{C}$ \\
$\mathrm{A}$
\end{tabular} & $\begin{array}{l}\text { A } \\
\text { C } \\
\text { P } \\
\text { P } \\
\text { C } \\
\text { A } \\
\text { C } \\
\text { C } \\
\text { A } \\
\text { C }\end{array}$ & $\mathbf{P}$ & $\begin{array}{l}\mathrm{R} \\
\mathrm{R} \\
\mathrm{R} \\
\mathrm{R} \\
\mathrm{R} \\
\mathrm{R}\end{array}$ & $\begin{array}{l}\mathrm{C} \\
\mathrm{R} \\
\mathrm{R}\end{array}$ & $\begin{array}{l}\mathrm{R} \\
\mathrm{P} \\
\mathrm{P} \\
\mathrm{P} \\
\mathrm{P} \\
\mathrm{P} \\
\mathrm{P} \\
\mathrm{P} \\
\mathrm{P}\end{array}$ & \begin{tabular}{|l|}
$R$ \\
$R$ \\
$C$ \\
$R$ \\
$C$ \\
$P$
\end{tabular} & $\begin{array}{l}\mathbf{R} \\
\mathbf{R} \\
\mathbf{R}\end{array}$ & $\begin{array}{l}P \\
R \\
R \\
R \\
R \\
R \\
\\
P \\
R \\
R\end{array}$ & $\mathrm{~T}$ & $\begin{array}{l}\mathrm{P} \\
\mathrm{P} \\
\mathrm{P} \\
\mathrm{R} \\
\mathrm{C} \\
\mathrm{C} \\
\mathrm{P} \\
\mathrm{C}\end{array}$ & $\mathbf{R}$ & $\begin{array}{l}\mathrm{R} \\
\mathrm{R}\end{array}$ & & & & & & \\
\hline 131.0 & 18 & $\begin{array}{l}2 \\
3 \\
4 \\
\mathrm{cc}\end{array}$ & \begin{tabular}{|c|}
$45-47$ \\
$102-109$ \\
$45-47$ \\
$97-104$ \\
$42-45$ \\
$99-106$ \\
$25-27$ \\
\end{tabular} & \begin{tabular}{|l} 
C \\
A \\
F \\
F \\
A \\
C \\
A \\
A
\end{tabular} & $\begin{array}{l}\text { G } \\
\text { G } \\
\text { M } \\
\text { M } \\
\text { G } \\
\text { G } \\
\text { G } \\
\text { G }\end{array}$ & \begin{tabular}{|l|}
2 \\
1 \\
3 \\
2 \\
2 \\
2 \\
2 \\
1
\end{tabular} & $P$ & $\begin{array}{l}\text { of } \\
\text { of } \\
\text { of }\end{array}$ & \begin{tabular}{l|} 
C \\
C \\
C \\
C \\
C \\
C \\
C \\
A
\end{tabular} & \begin{tabular}{|l|}
$\mathbf{R}$ \\
$\mathbf{R}$ \\
$\mathbf{R}$ \\
\end{tabular} & \begin{tabular}{|l|}
$\mathrm{C}$ \\
$\mathrm{A}$ \\
$\mathrm{P}$ \\
$\mathrm{P}$ \\
$\mathrm{C}$ \\
$\mathrm{C}$ \\
$\mathrm{A}$ \\
$\mathrm{A}$
\end{tabular} & $\begin{array}{l}\text { C } \\
\text { A } \\
\text { C } \\
\text { C } \\
\text { C } \\
\text { C } \\
\text { A } \\
\text { C }\end{array}$ & P & $\begin{array}{l}\mathbf{R} \\
\mathrm{R}\end{array}$ & $\mathrm{R}$ & $\begin{array}{l}\mathrm{P} \\
\mathrm{P} \\
\\
\mathrm{P} \\
\mathrm{P} \\
\mathrm{P} \\
\mathrm{P}\end{array}$ & $\begin{array}{l}\mathbf{P} \\
\mathbf{P}\end{array}$ & $\mathbf{R}$ & $\begin{array}{l}\mathrm{R} \\
\mathrm{R} \\
\mathrm{R} \\
\mathrm{R} \\
\mathrm{P} \\
\mathrm{P} \\
\mathrm{P}\end{array}$ & & \begin{tabular}{l|l} 
C \\
P \\
\\
R \\
$\mathrm{T}$ \\
$\mathrm{A}$
\end{tabular} & $\mathbf{P}$ & $\begin{array}{l}\mathrm{R} \\
\mathrm{R}\end{array}$ & & & & & & \\
\hline
\end{tabular}

top of the Olduvai Event (1.72 m.y. ago) (Berggren et al., 1980). The Quaternary was divided into two zones, one of which had three subzones. Twenty-seven climatic subdivisions were recognized in the Quaternary, and three were recognized in the Pliocene.

\section{Definition of Zones and Subzones}

Zones are named after the taxon that appears at its base. Subzones are named after the most frequently occurring taxon. The zones are discussed below from the most recent to the oldest deposits. Zones that encompass a taxon's entire range are called taxon range zones (TRZs); zones that span only part of a taxon's range are called partial range zones (PRZs).

\section{Globorotalia truncatulinoides Zone (TRZ)}

Base: first appearance of Globorotalia truncatulinoides Top: Recent (not defined)

This taxon appears suddenly, without any evident phylogenic transition from its presumed ancestor, $G$. tosaensis. The taxon appeared 880,000 to 900,000 yr. ago in the upper part of the Jaramillo Event. The zone is subdivided into three subzones, which are discussed below from top to bottom.

Turborotalita humilis Subzone (TRZ)

Base: first appearance of Turborotalita humilis Top: Recent (not defined) 
Table 2E. Distribution of planktonic foraminifers in Hole 548 (Cores 19-35).

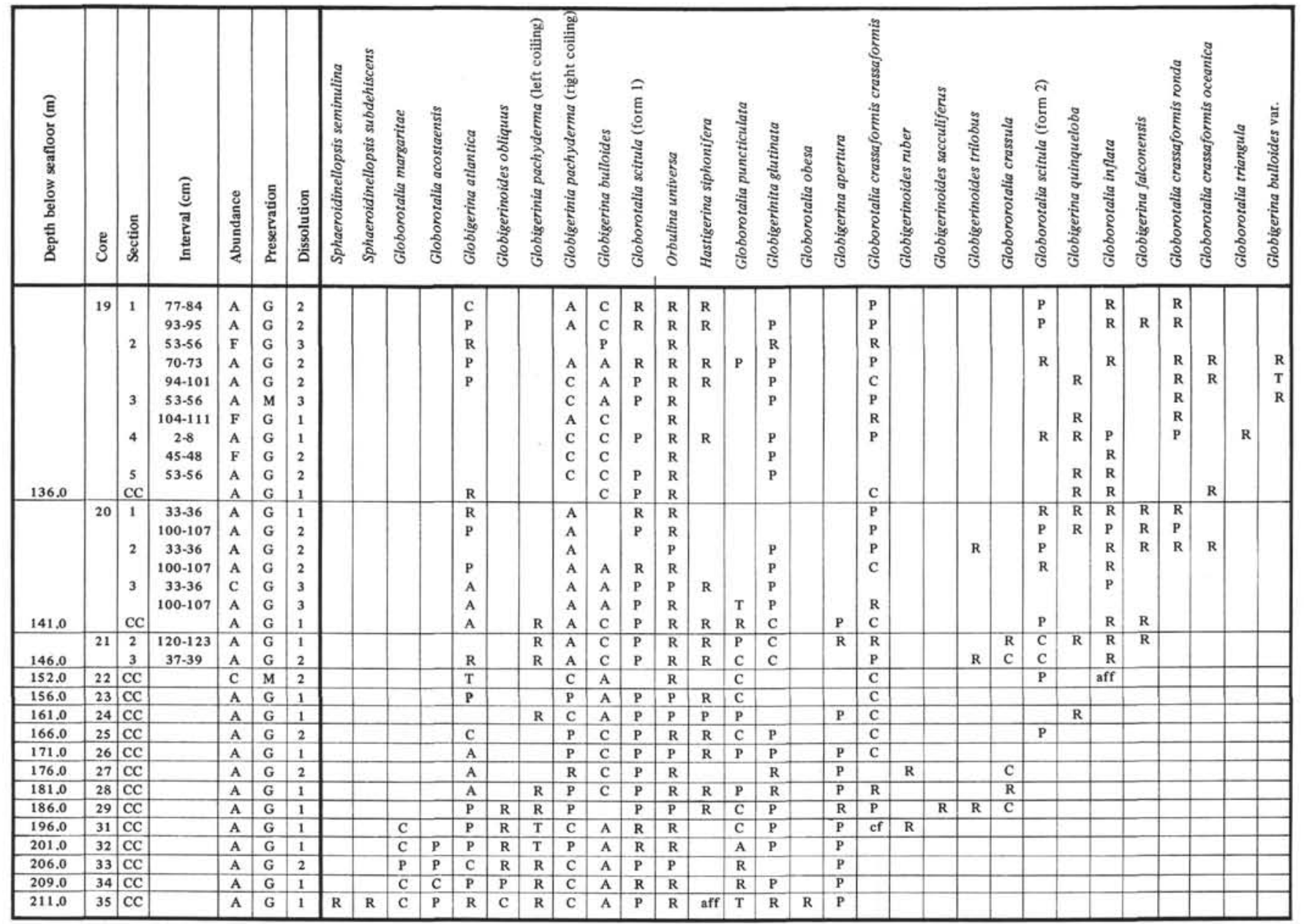

This small species, which is seen most often in size fractions between 150 and $63 \mu \mathrm{m}$, has a limited vertical distribution at the latitude of the Bay of Biscay. In the northeast Atlantic, T. humilis is present only in the upper part of the postglacial period, or Isotopic Stage 1 (Pujol, 1980).

\section{Globorotalia hirsuta Subzone (PRZ)}

Base: first appearance of Globorotalia hirsuta

Top: first appearance of Turborotalita humilis

G. hirsuta appears in the northeast Atlantic (Pujol, 1980 ) close to the Pseudoemiliania lacunosa last appearance datum, that is, approximately 470,000 yr. ago (Thierstein et al., 1977). This level is constant from the Azores Archipelago to Ireland in the regional distribution of the taxon.

\section{Globorotalia truncatulinoides Subzone (PRZ)}

Base: first appearance of Globorotalia truncatulinoides Top: first appearance of $G$. hirsuta

\section{Globorotalia inflata Zone (PRZ)}

Base: disappearance of Globigerina atlantica Top: first appearance of Globoratalia truncatulinoides
This zone includes the upper part of the Pliocene and the early Quaternary. The taxon begins to occur more frequently and attains its characteristic form in the middle of the zone, close to the base of the Olduvai Event. The first period of glaciation, which is reflected in a sudden and important increase in Arctic fauna (Globigerina pachyderma, left-coiling), also takes place during the Olduvai Event.

\section{Globigerina atlantica Zone (PRZ)}

Base: disappearance of Globorotalia margaritae Top: disappearance of Globigerina atlantica

Globoratalia crassaformis and G. crassula develop within this zone. The first $G$. inflata individuals evolve gradually from $G$. puncticulata. This zone is part of the late Pliocene. Two subzones were distinguished. They are described below from top to bottom.

\section{Globorotalia crassaformis ronda Subzone (PRZ)}

Base: first appearance of Globorotalia crassaformis ronda

Top: disappearance of G. atlantica

The last specimens observed of the $G$. puncticulata species were at the base of this subzone. The $G$. crassaformis group begins to develop at this level. 
Table 3. Distribution of planktonic foraminifers in Hole 549A (Cores 1-3).

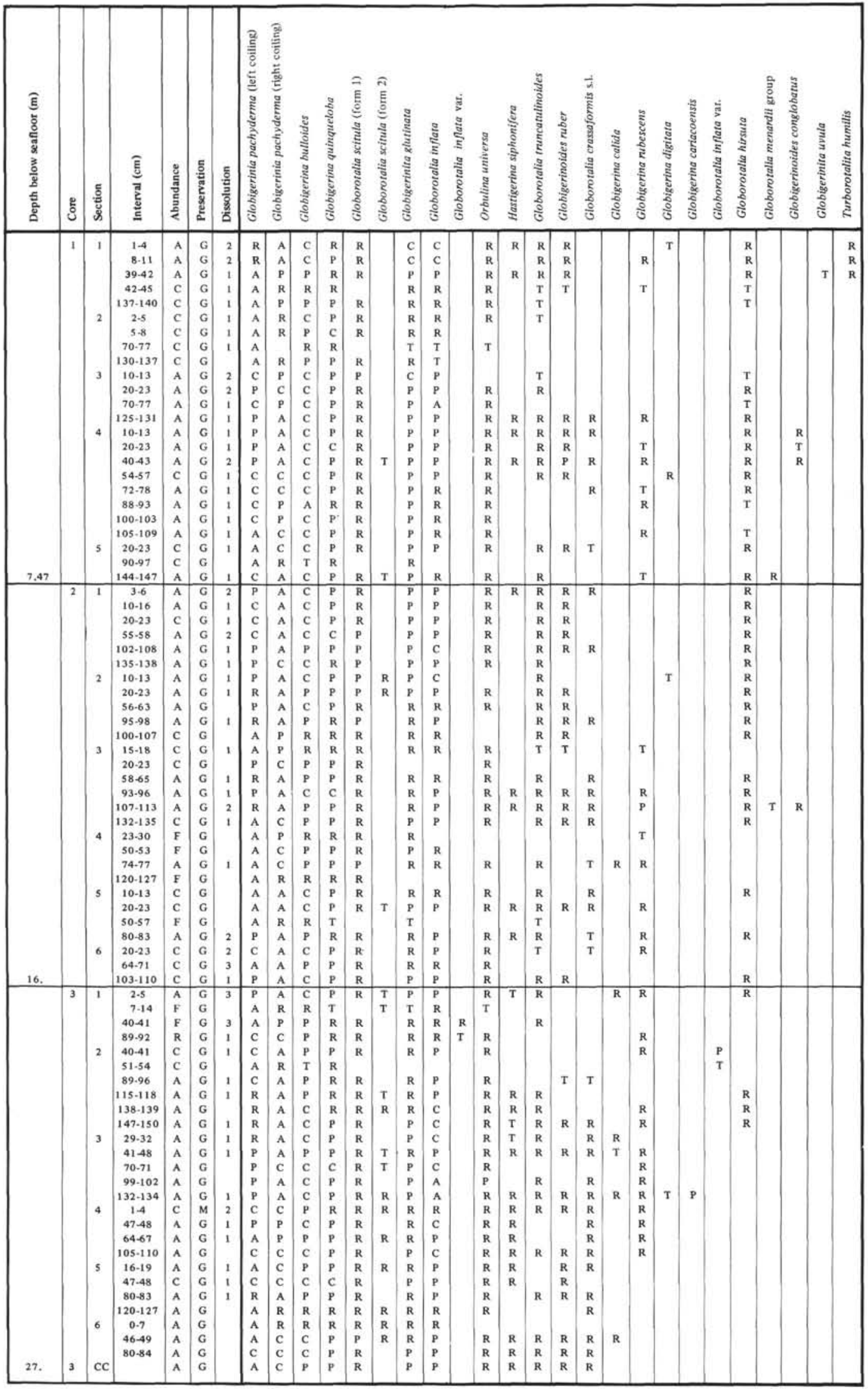

Note: The order in which species appear and their abundance, preservation, and dissolution are as defined in Table 2 . 
Table 4. Distribution of planktonic foraminifers in Hole 550 (Core 1).

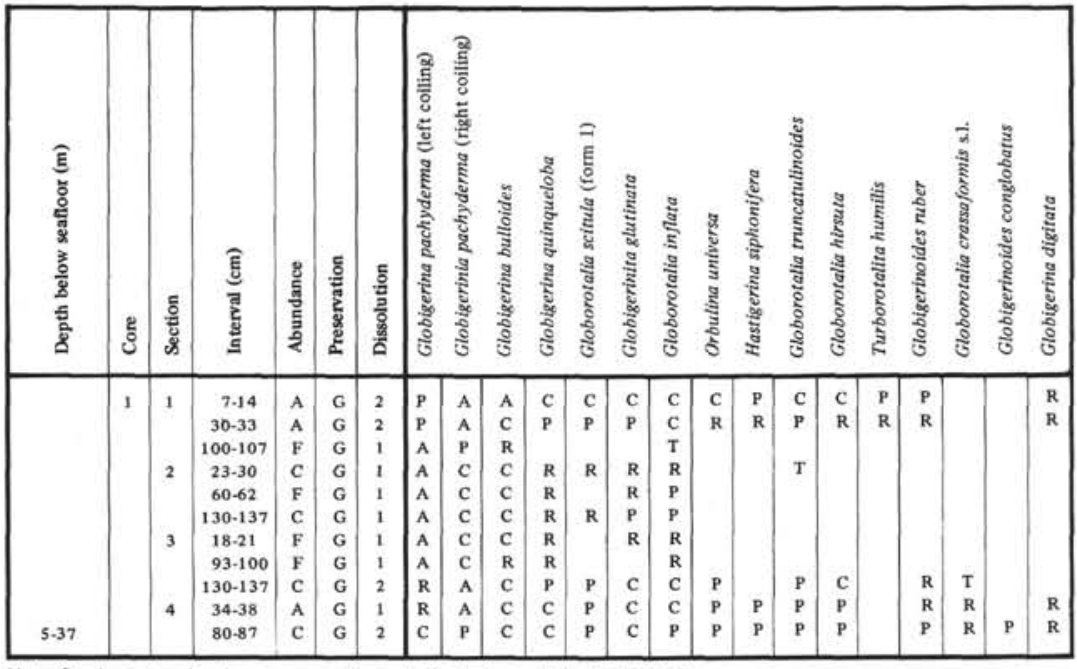

Note: Species order, abundance, preservation, and dissolution as defined in Table 2.

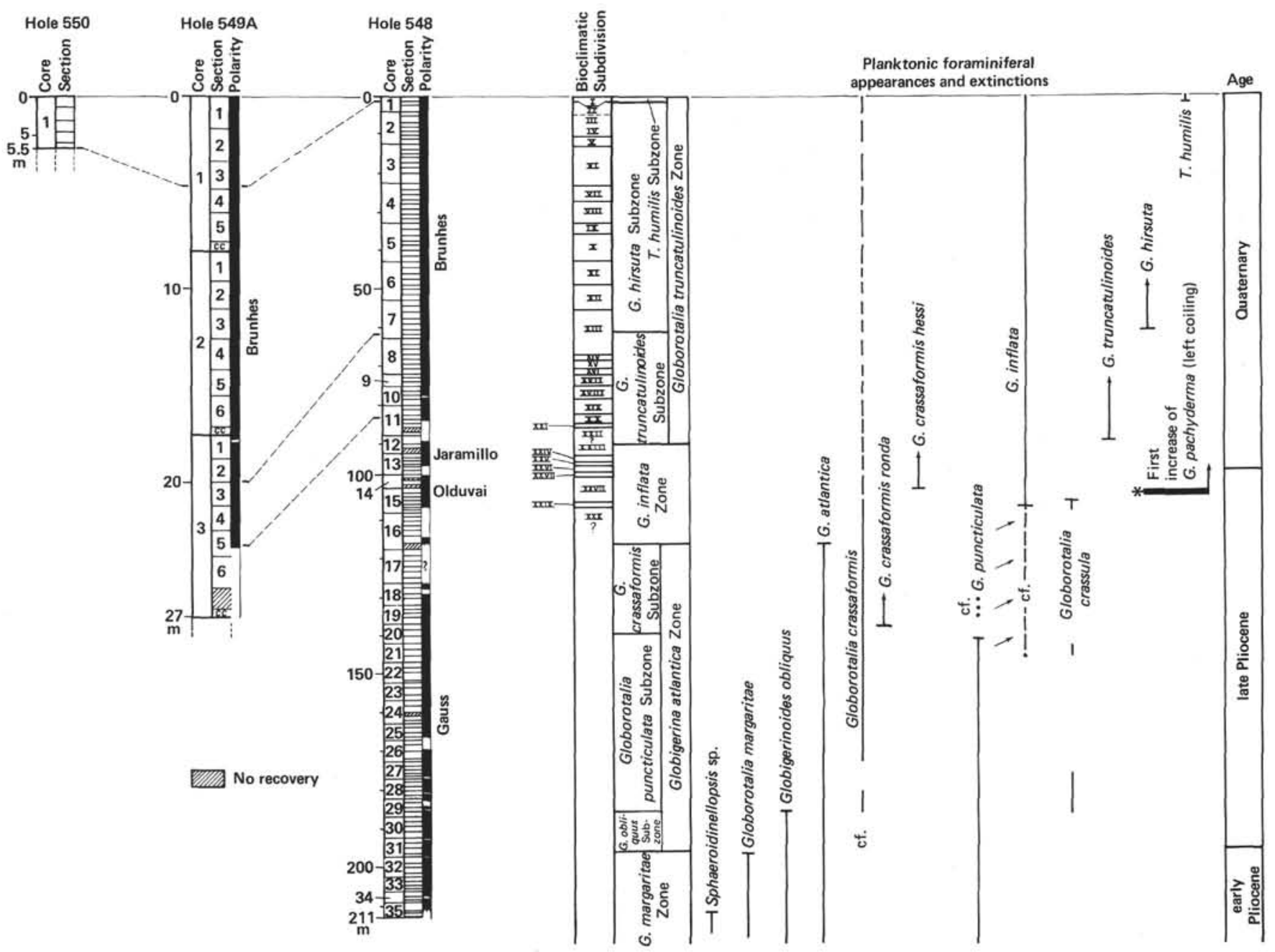

Figure 2. Biozonation of the Pliocene-Quaternary at Holes 548, 549A, and 550 with the planktonic foraminiferal datum levels. 


\section{Globorotalia puncticulata Subzone (PRZ)}

Base: last appearance of Globigerinoides obliquus $d a$

Top: first appearance of Globorotalia crassaformis ron-

G. aff. inflata appears at the top of this subzone and G. crassula at the base.

\section{Globigerinoides obliquus Subzone (PRZ)}

Base: disappearance of Globorotalia margaritae Top: disappearance of Globigerinoides obliquus

\section{Globorotalia margaritae Zone (taxon range not defined)}

Base: not observed

Top: disappearance of Globorotalia margaritae

This zone is assigned to the early Pliocene.

The characteristics of this biozonation are intimately related to the climatic conditions that prevailed over the North Atlantic during the Quaternary. The location of the sites near the center of the zone affected by the movement of the polar front makes the biozonation particularly interesting.

However, no satisfactory comparison can be made with the tropical and equatorial regions, where the hydrology was not influenced by the polar (cold) water. Equatorial zonations (Blow, 1969; Ericson and Wollin, 1956 and 1968; Bolli and Premoli Silva, 1973; Pujol and Duprat, 1984) cannot therefore be accurately correlated with the biozonation we have developed.

\section{Bioclimatic Subdivisons of the Quaternary}

Quaternary climatic variations correspond to variations in the volume of ice. In the North Atlantic, the building up and the spreading out of ice caused the movement of glaciers toward low latitudes, and consequently the displacement of Arctic microfauna. The farthest south the glaciers moved was about $42^{\circ} \mathrm{N}$ (CLIMAP Project Members, 1976).

The variations in climate are reflected on the Goban Spur in an increase in Arctic planktonic microfauna ( $G$. pachyderma, left-coiling). A high proportion of this form $(90-100 \%$ sinistral) suggests the proximity or presence of polar water near the Goban Spur. Conversely, a low proportion of this taxon and the simultaneous presence of subtropical microfauna reflect postglacial and/or interglacial conditions.

We used the microfauna at the Goban Spur to divide the Quaternary into 27 bioclimatic episodes or subdivisions. Episodes that resemble the climate of the present day (warm climatic stages) are marked by odd Roman numerals. Cold climatic stages are marked by even Roman numerals. All the episodes are essentially biologic subdivisions. Chronological correlations were made later on. Because of greater faunal variability, warm episodes proved easier to differentiate than cold episodes. Figure 2 and the figures presented for each site show the distribution of the bioclimatic subdivisions.

The following observations may be made about the bioclimatic subdivisions. First, from the pattern of re- cent deposits, it appears that the T. humilis Subzone corresponds or is equivalent to Subdivision I and that the $G$. hirsuta Subzone corresponds to Subdivisions II to XIII (upper part). Subdivisions II, III, and IV correspond to the last glacial stage. Caralp (1971) and Caralp et al. (1974) investigated the equivalent period in the Bay of Biscay, although they used the usual Alpine nomenclature. The available samples permit only a weak differentiation of these episodes, although Episode III can be recognized as an interstage.

The base of Subdivision V is characterized by the disappearance of Globigerinoides conglobatus and the presence of Globorotalia crassaformis. The disappearance of both taxa was detected during the last interglacial period (Isotopic Stage 5) throughout the northest Atlantic at latitudes above $42^{\circ} \mathrm{N}$ (Pujol, 1980).

Subdivision VII is distinguished by a temporary increase in the proportion of sinistral $G$. truncatulinoides. Pujol, Duprat, et al. (1974) designated this period in the Bay of Biscay Episode O.

Subdivision IX is characterized by the brief presence of the tropical species G. menardii. The presence of this species, which was also observed in the Bay of Biscay, is worthy of mention because this is the only time this tropical species invades such high latitudes during the Quaternary. A similar invasion takes place close to the Azores Archipelago during Isotopic Stage 9 (Pujol, 1980).

Subdivision XI is difficult to term a warm stage. There is a slight dominance of the sinistral population of $G$. truncatulinoides.

Some forms considered a variety of $G$. inflata occur in very limited amounts during Subdivision XII.

The middle part of Subdivision XIII is marked by the appearance of Globorotalia hirsuta.

The subdivisions above are correlated respectively to Isotopic Stages 1 to 13 , which could be recognized in Hole 548 in Cores 1 to 8 and in Hole 549A in Core 1 to Core 3, Section 2 (Vergnaud Grazzini et al., this volume).

The G. truncatulinoides Subzone includes the lower part of Subdivision XIII to the upper part(?) of Subdivision XXIII.

Warm Episodes XV, XVII, XIX, and XXI (upper part) are normally characterized by a subtropical microfauna, the most significant species of which are $G$. truncatulinoides and $G$. crassaformis. The $G$. crassaformis species is represented by the subspecies ronda and hessi. Subdivision XIX is found at the Brunhes/Matuyama paleomagnetic reversal.

G. truncatulinoides appears in Subdivisions XXII to XXIII, which together may be considered synchronous to the base of the $G$. truncatulinoides Zone.

The base of the Quaternary (top of the Olduvai Event) is included in the upper part of the G. inflata Zone. Subdivisions XXIII(?) to XXVII are also part of the G. inflata Zone. Subdivision XXVIII, which is located within the Olduvai Event, corresponds to the first cold episode and is characterized by the frequent occurrence of Arctic microfauna. The first terrigenous evidence of icerafting was observed below this limit. 
It was not possible to compare or correlate Subdivisions XIV to XXX with the isotopic stages recognized by Vergnaud Grazzini et al. (this volume).

\section{HOLE 548}

In Hole 548, continuous coring with the hydraulic piston corer resulted in the nearly complete recovery of an excellent Quaternary and Pliocene sequence $211 \mathrm{~m}$ thick (Fig. 3 and Table 2). Thirty-five cores were recovered. The top of the Olduvai Event was located at $101.1 \mathrm{~m}$ sub-bottom (Core 15, Section 1).

The Pliocene-Quaternary sequence consists of three lithologic units (site chapter, this volume). The first unit as one proceeds from top to bottom (Cores 1-8) is made up of alternating olive gray marly calcareous and nannofossil ooze. In Subunit 1a (from the surface to Core 7 , Section 5), carbonate content varies widely (from 50 to $10 \%$ ), with cyclical characteristics (Fig. 3A). In Subunit $1 \mathrm{~b}$ (Core 7, Section 5 through Core 8), the range of the cyclical variation is lower.

Lithologic Unit 2 ranges from Core 9 through Core 15. The sediments consist of reddish or grayish clay and foraminifer-nannofossil oozes. The carbonate content is relatively constant in Cores 9 and 10 (Fig. 3B). Carbonate content decreases to a low value in Core 11, Section 4, close to the Brunhes/Matuyama paleomagnetic reversal. The underlying deposits undergo rather wide variations. Probably because of a decrease in accumulation rate, cycles are more difficult to identify than in the upper part of Unit I.

A third lithologic unit (Cores 16-35) consists of homogeneous greenish gray bioturbated nannofossil ooze. Variations in carbonate content are rapid down to Core 20 (Fig. 3C). Below Core 20 the more scattered sampling does not allow the documentation of such variations as those above. Nevertheless, it is possible that the lithology within Unit 3 is uniform.

The maximum values of carbonate content correspond to maxima in the coarse (sand) fraction, and minimum values of carbonate content correspond to maxima in the fine grain size fraction (Fig. 3). There is a significant change in grain size in the Pliocene-Quaternary sequence at the base of Core 15 . Sediments of very fine grain size predominate from Core 15 to 35 ; the sediments are practically free of a coarse fraction, in contrast to the coarse sediments in the upper part of the hole (Cores 1-15).

All the biostratigraphic zones, subzones, and bioclimatic subdivisions described in the previous section were recognized in Hole 548. The lowest Quaternary sediments occur in Sample 548-15-1, 110-112 cm (the top of the Olduvai Event). The base of the Globorotalia truncatulinoides Zone, which is defined as the appearance of the taxon, occurs in Core 12, Section 1, 129-130 cm (during the Jaramillo Event). G. hirsuta occurs for the first time during the Brunhes Event (Core 7, Section 7, $10-13 \mathrm{~cm}$, Fig. 3A). Finally, Turborotalita humilis appears only in Core 1, Section 1, 0-3 cm.

The thickness of the $G$. hirsuta Subzone indicates a rapid sedimentation rate that could be the result of supplementary supply from the continental shelf. The base of the subzone is marked by a sharp change in both lithology and carbonate content.

The disappearance of Globigerinoides conglobatus within Core 3, Section 2, 76-79 cm, occurs within climatic Subdivision V; Globorotalia menardii occurs only in climatic Subdivison IX (Core 5, Section 2, 77-78 cm), and G. inflata var. occurs only in climatic Subdivision XII (Core 7, Section 4).

The population of $G$. hirsuta is predominantly rightcoiling, but at the top of the hole (climatic Subdivisions II, III, and IV) it becomes predominantly left-coiling. It is dextral from its appearance in Subdivision XIII to Subdivision V. The population of G. truncatulinoides is predominantly right-coiling, although some sinistral pulsations or coiling ratio changes were noted in climatic Subdivisions V, IX, XI, and XIII.

In the G. truncatulinoides Subzone (Fig. 3B) the sedimentation rate is lower than in the previous subzone. The planktonic foraminiferal assemblages during both warm and cold episodes are similar to the assemblages in the G. hirsuta Subzone. However, there is an important increase in the occurrence of $G$. pachyderma (rightcoiling) in Cores 11 and 12, an increase that defines a separate assemblage. The cold climatic subdivisions in this interval (XX and XXII) are characterized not by the Arctic species G. pachyderma (left-coiling) but by the subarctic dextral population. This assemblage persists until the upper part of the G. inflata Zone (Jaramillo Event). The Quaternary base, which is included in the upper $G$. inflata Zone, is characterized by cyclical variations in fauna in which G. pachyderma (left-coiling) again marks the cold episodes (Figs. 3B and 4). The first incursion of a typical polar fauna occurs in Core 15 , Section 4, that is, largely below the top of the Olduvai Event. The grain size of the sediment increases in Sample $548-15-6,120-123 \mathrm{~cm}$.

The transition between Pliocene and Quaternary sediments ( $G$. inflata Zone) was observed between Core 16, Section 1, 140-141 cm and Core 16, Section 5, 10-11 $\mathrm{cm}$. It is characterized by very fine-grained sediments containing very small microfossils. $G$. inflata becomes abundant and morphologically typical above Core 25 , Section $6,30-33 \mathrm{~cm}$; below that level it is quite rare and noncharacteristic.

Only the core catchers were analyzed in the Pliocene sequence from Cores 16 to 20 (Fig. 3C). In this sequence the gradual evolution between $G$. puncticulata and $G$. inflata takes place.

$G$. crassula appears within the $G$. puncticulata Subzone. As shown in Figure 3D, the quite restricted range of this species is marked by a change from dominantly dextral at the bottom (Core 29) to dominantly sinistral at the top (Core 27). The opposite trend takes place during the Pliocene in the $G$. crassaformis population.

Finally, G. margaritae (always left-coiling) is found from the base of the hole to the core catcher for Core 31; it characterizes early Pliocene sediments.

\section{HOLE 549A}

The three cores examined from Hole 549A represent a Quaternary sequence $27 \mathrm{~m}$ long that corresponds to 


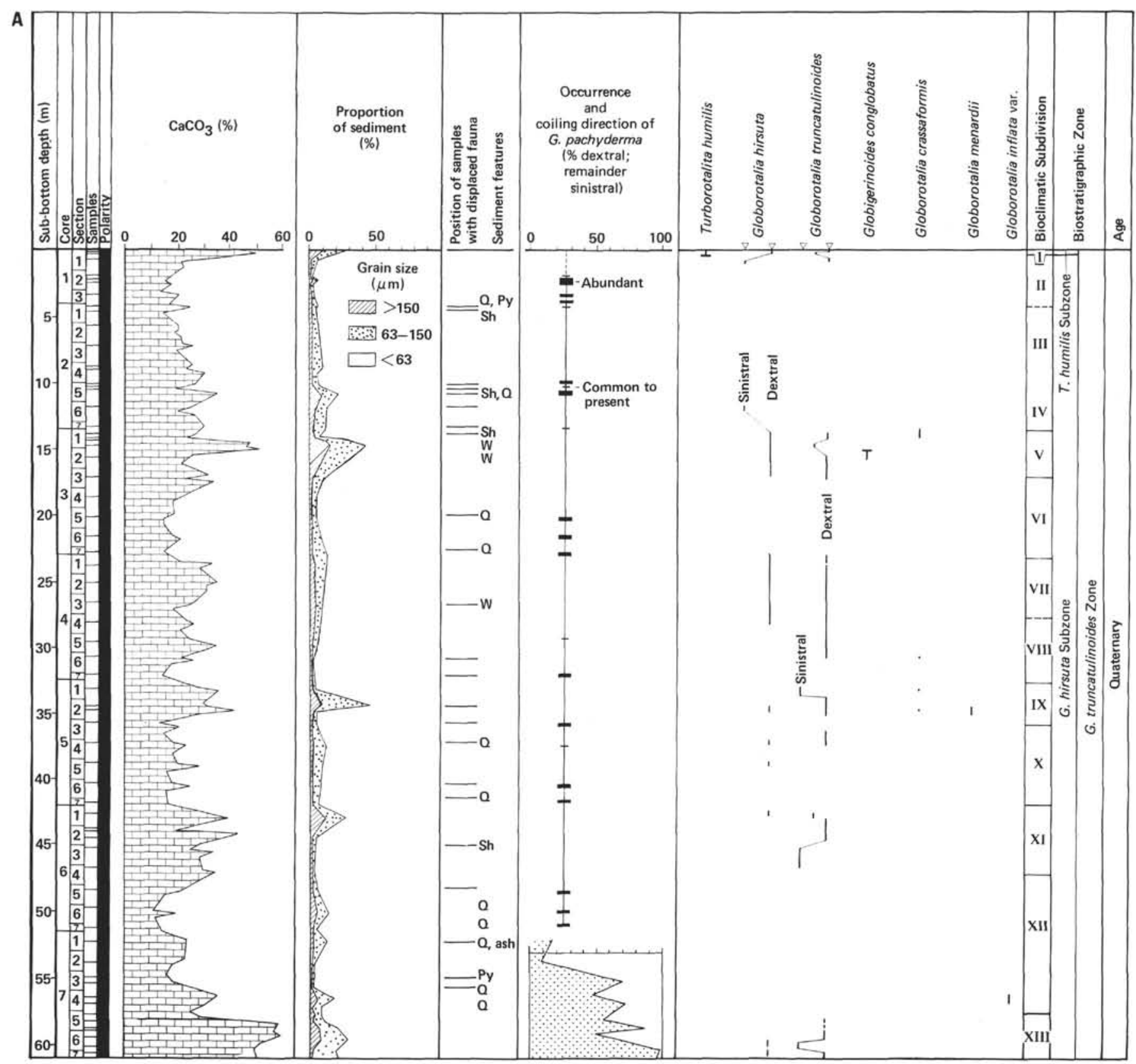

Figure 3. Analysis results for Hole 548. A. Cores 1 to 7. B. Cores 8 to 16. C. Cores 17 to 25 . D. Cores 26 to 35 . Py $=$ pyrite, Q $=$ quartz, Sh $=$ shell fragments, $\mathrm{W}=$ winnowed. The occurrence of species that appear in both left- and right-coiling forms is shown by a line that moves left and right according to the proportion of individuals of these types within the population.

Lithologic Unit 1 (site chapter, this volume). The sequence comprises marly calcareous nannofossil and foraminifernannofossil oozes. The Brunhes/Matuyama paleomagnetic reversal is located betwen 24 and $24.79 \mathrm{~m}$ sub-bottom (in Core 3, Section 5, between $50-52 \mathrm{~cm}$ and 129 $131 \mathrm{~cm}$; Townsend, this volume). Carbonate content varies cyclically from 60 to $10 \%$. The peaks in carbonate content are directly associated with peaks in the coarse sediment fraction, which in turn comprises mostly planktonic foraminiferal shells. A sudden lithlogic and microfaunal change between Cores 2 and 3 suggests the existence of a sedimentary gap. The Globorotalia trun- catulinoides Zone and the Turborotalita humilis, G. hirsuta, and $G$. truncatulinoides subzones have been determined (Fig. 5, Table 3).

The T. humilis Subzone is limited to Sample 549A-1$1,39-42 \mathrm{~cm}$.

The first occurrence of $G$. hirsuta was observed in Core 3, Section 2, 147-150 cm. It was not possible to determine the first occurrence of $G$. truncatulinoides.

An examination of the coiling direction of G. pachyderma from the top of this site shows a succession of warm episodes (in which there is an abundance of rightcoiling forms) and cold episodes (in which there is an 


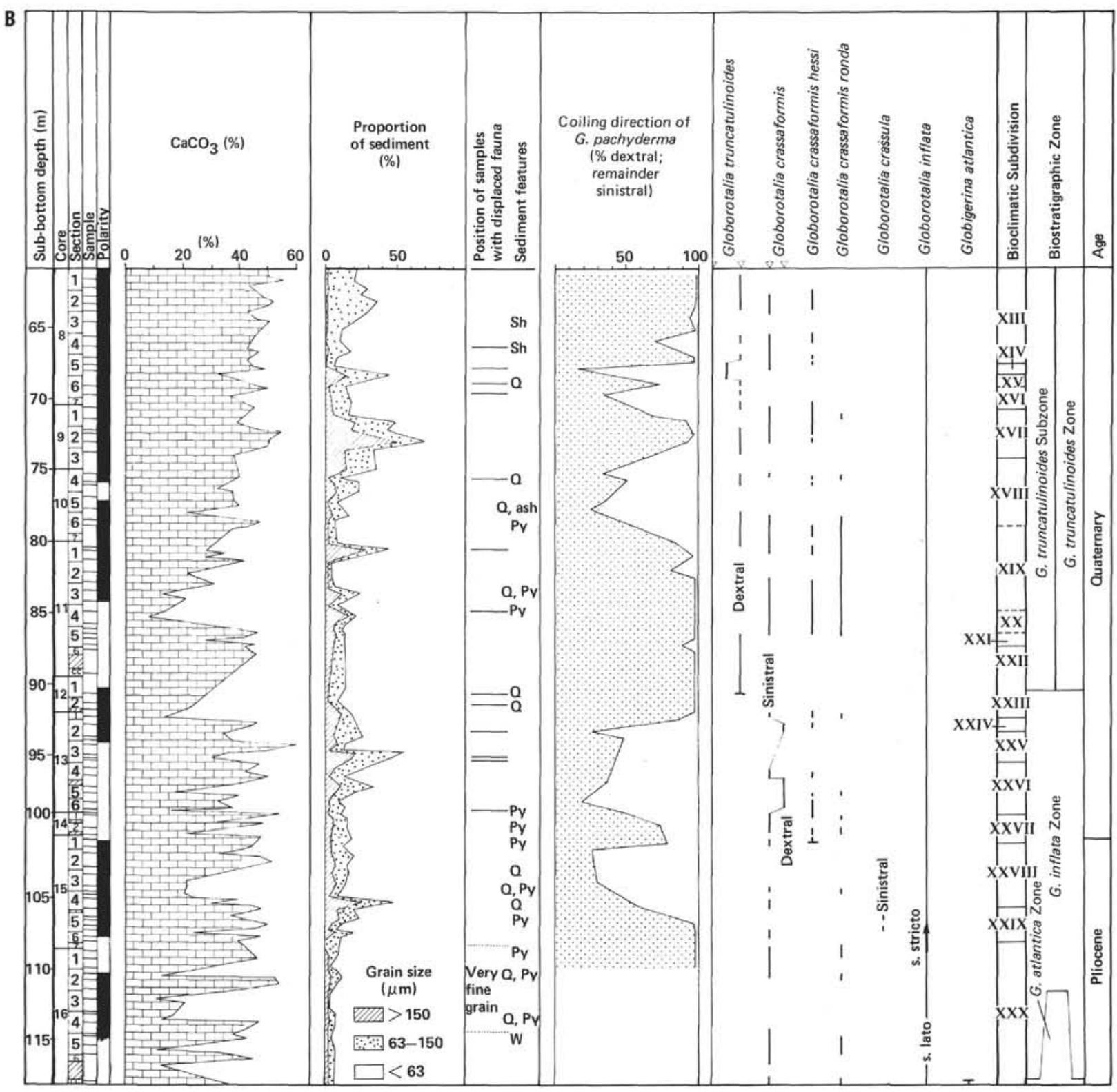

Figure 3. (Continued).

abundance of left-coiling forms). The warm episodes correspond perfectly to the peaks in carbonate content.

Thirteen bioclimatic subdivisions were distinguished within the $T$. humilis and G. hirsuta subzones. The hypothetical gap between Cores 2 and 3 mentioned above is reflected in a sudden change in faunal assemblage that takes place between Subdivisions XI and XII. It was difficult to determine the boundary between Subdivisions XII and XIII. The occurrence of $G$. inflata var. in Core 3 , Section 2 from $40-41 \mathrm{~cm}$ to $51-54 \mathrm{~cm}$ led us to locate Subdivision XII at these levels.

The disappearance of Globigerinoides conglobatus was detected in Core 1, Section 4, 10-13 cm. Globorotalia menardii occurs punctually in Core 2, Section 3, 107$113 \mathrm{~cm}$. G. hirsuta is right-coiling in climatic Subdivision I and throughout most of the rest of its range; it is dominantly sinistral only in Subdivisions II, III, and IV.
For the G. truncatulinoides species, three sinistral pulsations of the dominantly right-coiling population were observed in climatic Subdivisions VII, XI, and XIII.

The location of the Brunhes/Matuyama paleomagnetic reversal suggests that the sediment accumulation rate diminishes below the base of the G. hirsuta Subzone (Subdivision XIII), or approximately $470,000 \mathrm{yr}$. ago. The fluctuations in the coiling direction of $G$. pachyderma allow us to define Subdivisions XIV to XXI but without any accuracy.

\section{HOLE 550}

Only one surficial core $5.5 \mathrm{~m}$ long was recovered from Hole 550 (Fig. 6). The hole was at a water depth of 4432 $\mathrm{m}$, and the sediments consist of Quaternary foraminifer-nannofossil oozes. 


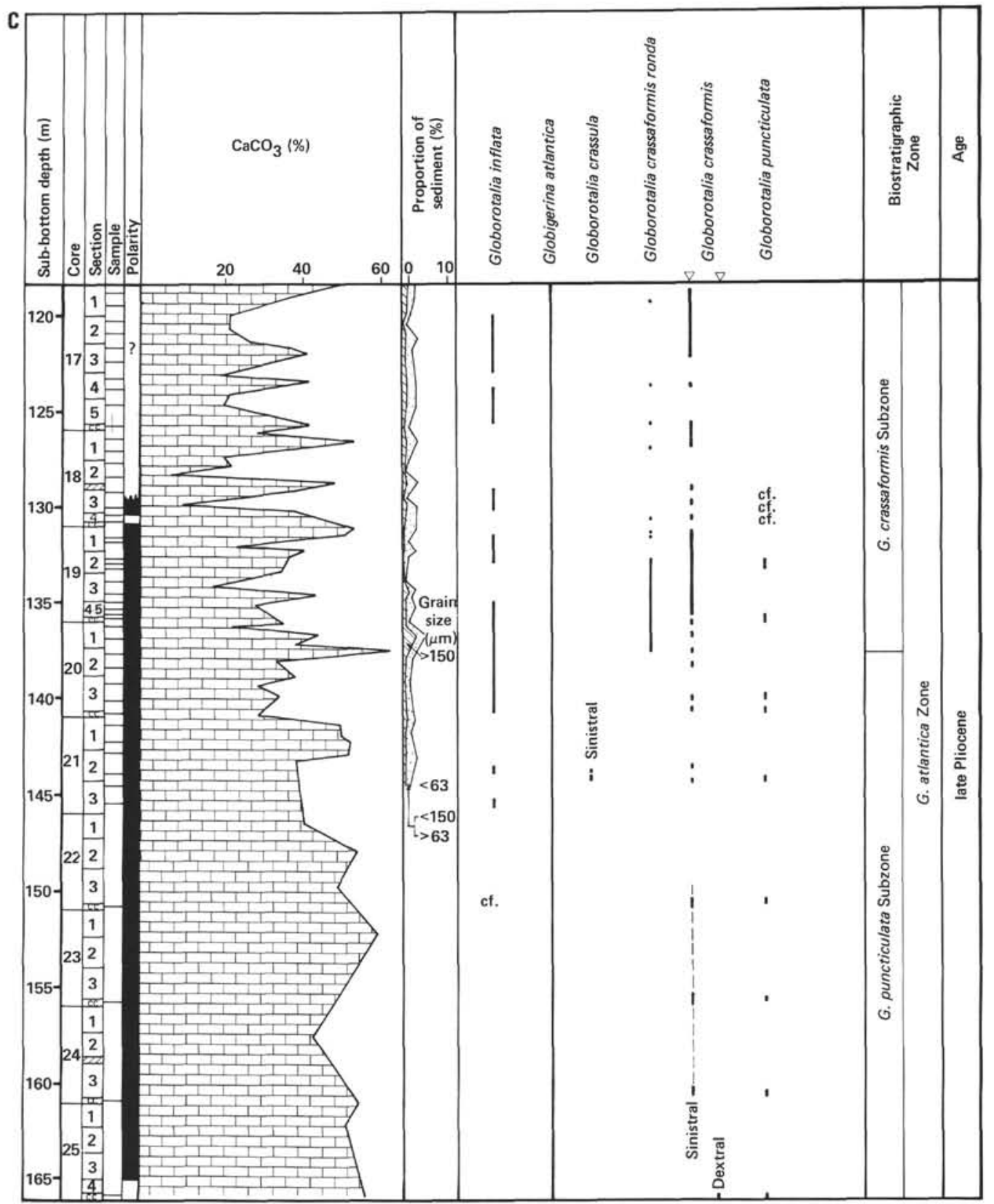

Figure 3. (Continued).

The Turborotalita humilis Subzone could be distinguished from the upper part of the Globoratalia hirsuta Subzone within the G. truncatulinoides Zone. In all, the first five climatic subdivisions could be recognized.

The T. humilis Subzone (climatic Subdivision I) extends into the uppermost sediments. The subzone corresponds to a peak in carbonate content.

Subdivision $\mathrm{V}$ is characterized by the occurrence within the faunal assemblage of $G$. truncatulinoides, G. hirsuta, G. crassaformis, and Globigerinoides conglobatus.

Subdivisions II, III, and IV were inferred from the observations above.

\section{SEDIMENTATION RATES}

Average sedimentation rates were calculated from the preliminary chronological framework shown in Figure
7. In Hole 548 , important variations were observed between the late (Brunhes) and early Quaternary. A frequent and substantial displacement of sediments from the continent occurred during the late Quaternary (Caralp, this volume). The lower Quaternary sediments, on the other hand, are relatively condensed. The average sedimentation rate of $11.5 \mathrm{~cm} / 10^{3} \mathrm{yr}$. from the late Quaternary of Hole 548 corresponds to the fairly high rate of sedimentation observed elsewhere in the North Atlantic. In Hole $549 \mathrm{~A}$ the average sedimentation rate of $3 \mathrm{~cm} /$ $10^{3} \mathrm{yr}$. reflects strong sediment condensation during the Brunhes period. The estimated sedimentation rates in Hole 549A since 470,000 yr. ago and in Hole 550 are similar (Fig. 8) and represent a pelagic sedimentation rate that is normal for this region. Thus, the sediments that record the climatic cycles most accurately belong 


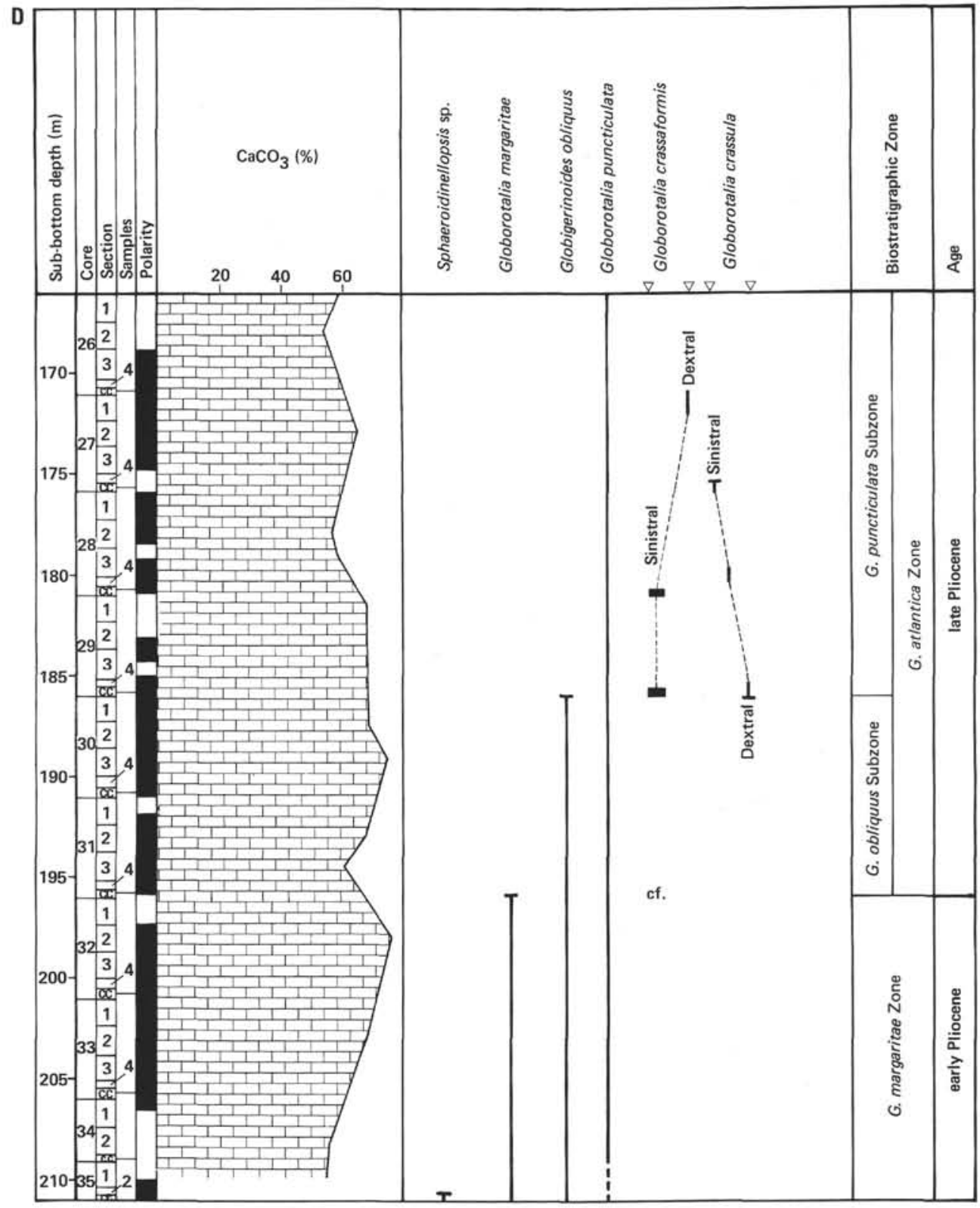

Figure 3. (Continued).

mainly to the late Quaternary between the Jaramillo period and the present. The recognition of the climatic cycles between the Olduvai and Jaramillo periods, an interval of fairly condensed sediments $\left(1.6 \mathrm{~cm} / 10^{3} \mathrm{yr}\right.$. in Hole 548) is problematic.

\section{CORRELATION AND CHRONOLOGY OF LEG 80 SITES}

Micropaleontological and lithologic data were used to develop the correlation between Holes 548, 549A, and 550 that is summarized below and in Figure 2.

Turborotalita humilis occurs in the uppermost part of the Quaternary sequence. This species defines an upper subzone within the Globorotalia truncatulinoides Zone. Climatic Subdivision I may be considered part of the postglacial period. A comparative study based on the surveys carried out in the Bay of Biscay (Caralp et al.,
1974; Pujol, 1980) permitted the correlation of the $T$. humilis Subzone with Isotopic Stage I, in particular its upper part (down to the base of $I_{B}$ ), which is dated as being 8000 yr. old (Duplessy et al., 1981).

The disappearance of Globigerinoides conglobatus in Subdivision V was observed in the Bay of Biscay and the northeast Atlantic at the base of Isotopic Stage 5 (an interval of maximum deglaciation). This event was dated as occurring 125,000 yr. ago. It suggests that Subdivisions II, III, and IV are part of the last glacial period. The occurrence of a sinistral Globorotalia hirsuta population (Figs. 3A and 5) confirms this hypothesis (Pujol, 1975; Pujol and Duprat, 1977). Therefore, Subdivision V is correlated with Isotopic Stage 5. Further evidence of a warm interglacial period in Subdivision $\mathrm{V}$ is the brief occurrence of $G$. crassaformis and a pulsation toward a left-coiling $G$. truncatulinoides population in Hole 


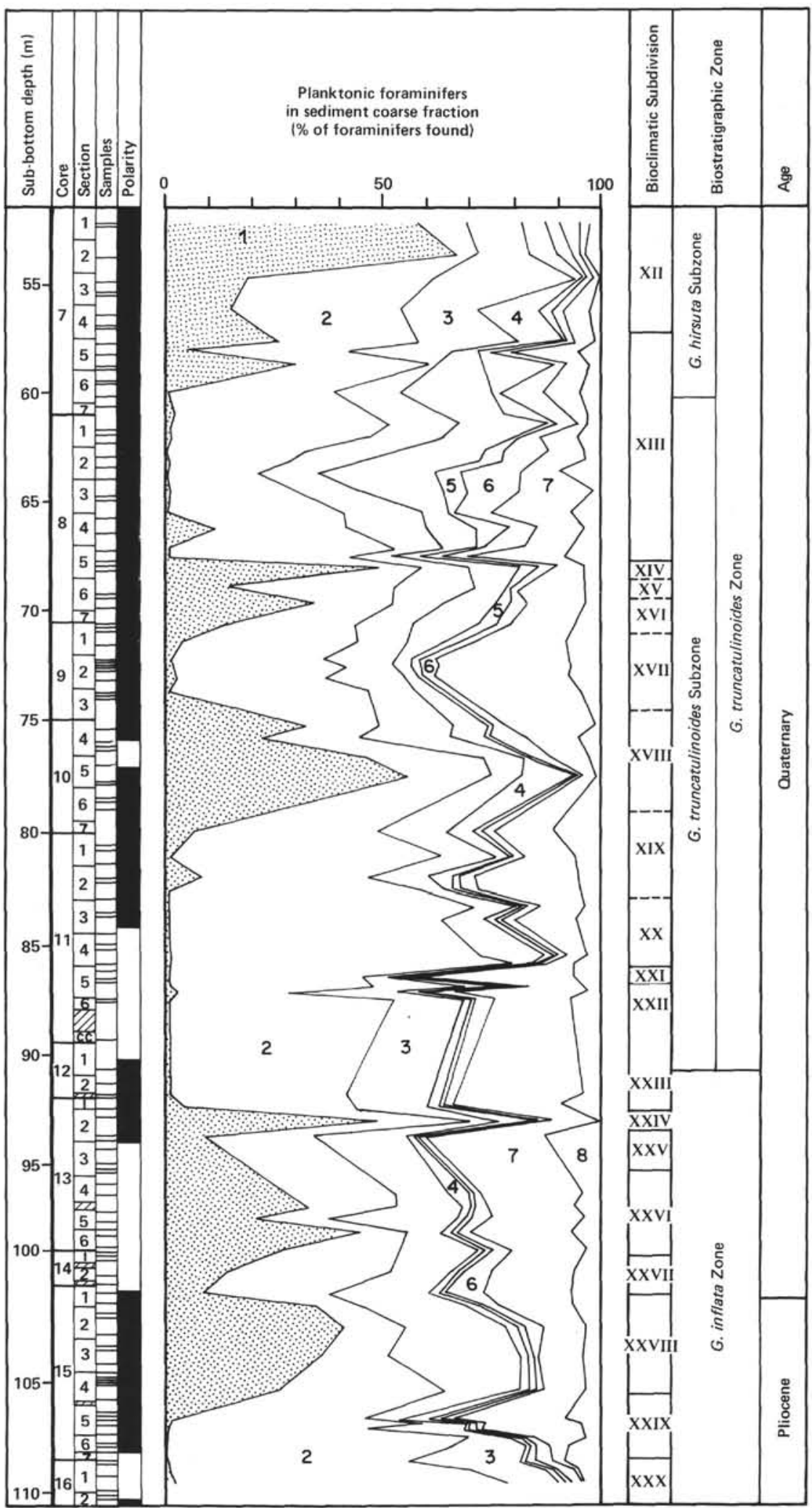

Figure 4. Cumulative percentage of planktonic foraminifers in sediment coarse fraction $(>150 \mu \mathrm{m})$. Hole 548, Cores 7 to 16 and comparison with biostratigraphy. $1=$ Globigerina pachyderma (left-coiling); $2=$ G. pachyderma (right-coiling); $3=$ G. bulloides; $4=$ G. quinqueloba; $5=$ Globorotalia scitula $; 6=$ Globigerinita glutinata; $7=$ Globorotalia inflata; 8 = other species. Note the first increase of left-coiling Globigerina pachyderma in Core 15, Section 4 and the development of right-coiling G. pachyderma in Cores 12 and 11 . 


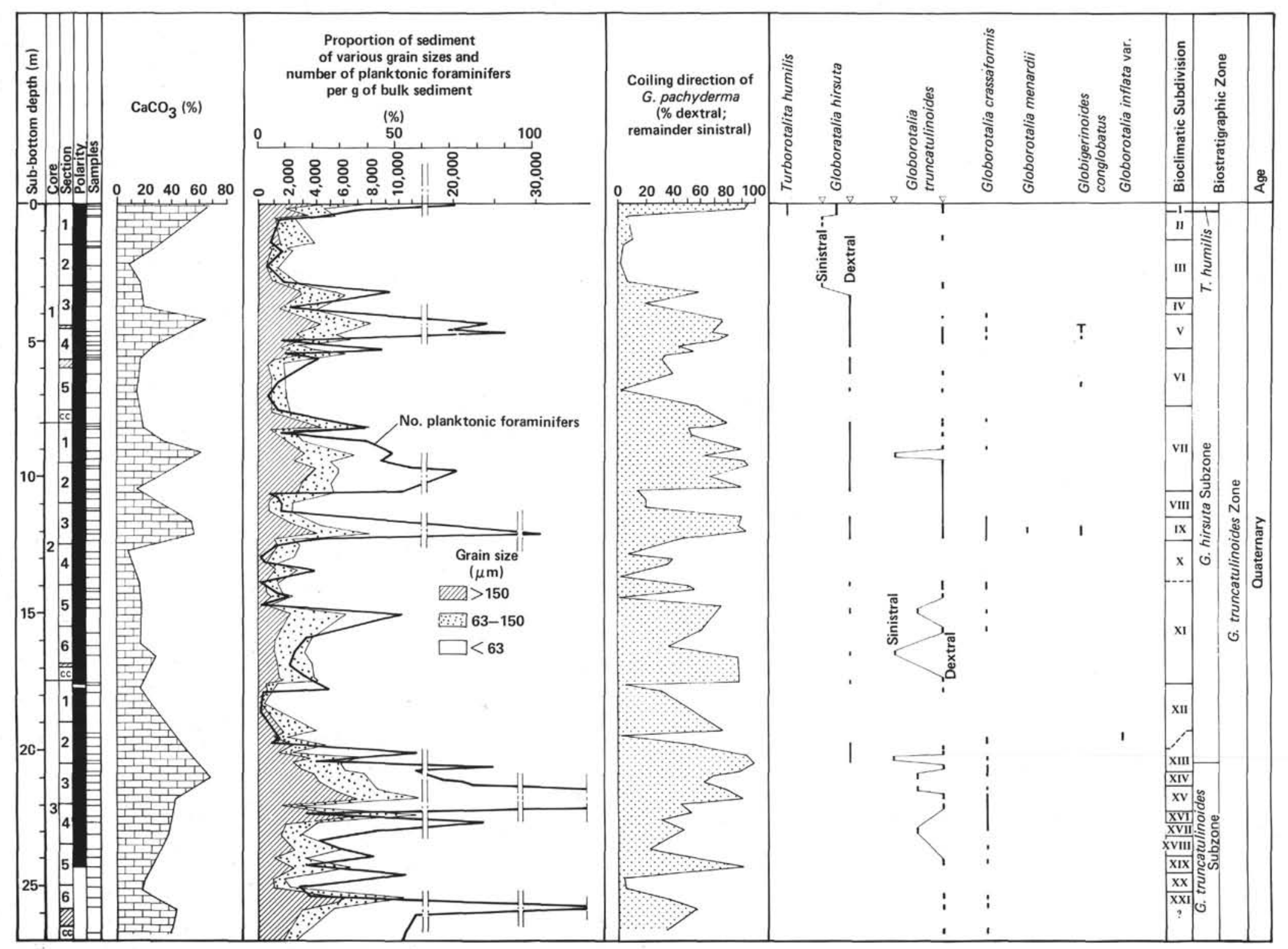

Figure 5. Analysis results for Hole 549A (Cores 1-3). 


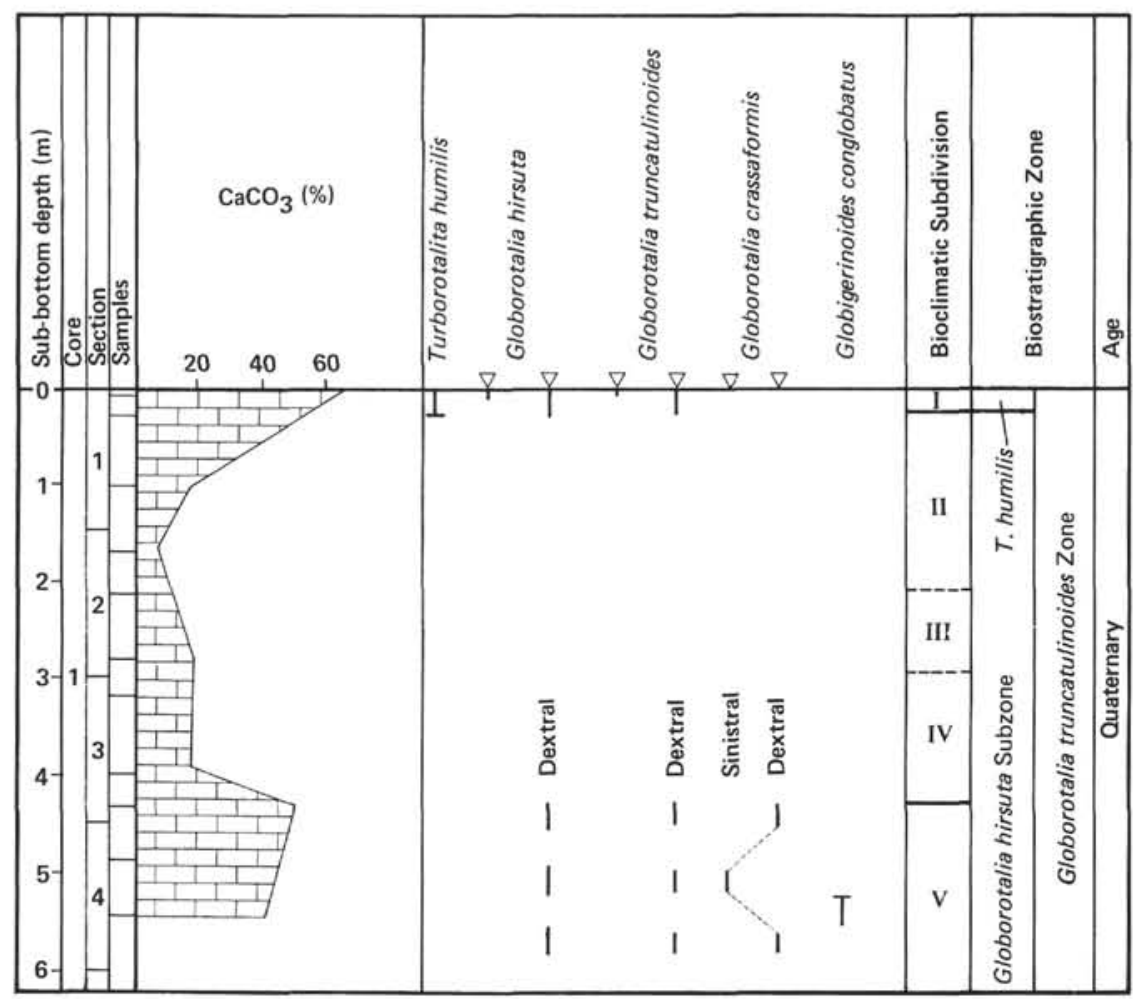

Figure 6. Analysis results for Hole 550 (Core 1).

548 (Fig. 3A). This pulsation was dated as occurring 105,000 to 110,000 yr. ago by extrapolating the sedimentation rate from an isotopic analysis of the Bay of Biscay and a region near Ireland (Pujol, 1980).

In Hole 549A, Subdivision VII is characterized by a sinistral pulsation of G. truncatulinoides (Fig. 5). Similar observations were made of numerous samples acquired between the Azores Archipelago and the coast of Ireland at Isotopic Stage 7 (Pujol, 1980).

At these latitudes, the presence of G. menardii appears to be characteristic of Isotopic Stage 9 (Pujol, 1980). This indicator could be given an age of about 300,000 yr.

The dominantly right-coiling $G$. truncatulinoides population in Subdivison XI has already been noted. Boundaries for this subzone can be defined only by using previously acquired data.

The first occurrence of $G$. hirsuta probably takes place in a subdivision equivalent to Isotopic Stage 13. The first occurrence of $G$. hirsuta was similar to this from the Azores to Ireland (Ruddiman and McIntyre, 1976; Pujol, 1980). This datum serves to emphasize the transition between two different environments within the Brunhes period (Caralp, this volume). The transition period is also characterized by the brief presence of $G$. inflata var.

The Brunhes/Matuyama reversal, which was dated as occurring 730,000 yr. ago by Berggren et al. (1980), permitted the dating of Subdivision XIX and its probable correlation with Isotopic Stage 19. Between this reversal and the Jaramillo Event, climatic Subdivisions XIX, XX, and XXII to XXIII reflect a surficial hydrological envi- ronment distinguished by a high percentage of Globigerina pachyderma (right-coiling).

The first appearance of Globorotalia truncatulinoides occurs in Subdivision XXIII in the upper part of the Jaramillo Event, which is dated as occurri.g approximately 900,000 yr. ago. This estimate of age, which was proposed by Poore and Berggren (1975) after DSDP Leg 12 (Site 116; Hatton Rockall Basin) and used by Backman (1979) and Blanc (1981), needs to be reconsidered.

The first glaciation, which is reflected in the occurrence of the Arctic fauna (Globigerina pachyderma, leftcoiling) takes place within the heart of the Olduvai Event. It reveals the existence of Subdivision XXVIII in the Globorotalia inflata Zone. The glaciation is, therefore, older than the base of the Quaternary (top of the Olduvai Event). The first evidence of an increase of the coarse terrigenous residue is detected at the base of the Olduvai Event (about 1,880,000 yr. ago).

At the base of the G. inflata Zone (Fig. 3B), which occurs within the Pliocene, an interval may be distinguished on the basis of the fine grain size of its sediments. According to the definition we have given to the G. inflata Zone, this interval may be considered a Pliocene/Quaternary transition zone. The microfaunal assemblage and its patterns (Fig. 4) look very much like those discussed by Poore and Berggren (1975) at Site 116 (DSDP Leg 12).

The Pliocene sediments were sampled less frequently than the Quaternary sediments, so our analysis of them is less detailed. The analysis was performed primarily to acquire information about the period immediately preceding the Quaternary so we could assess the character- 


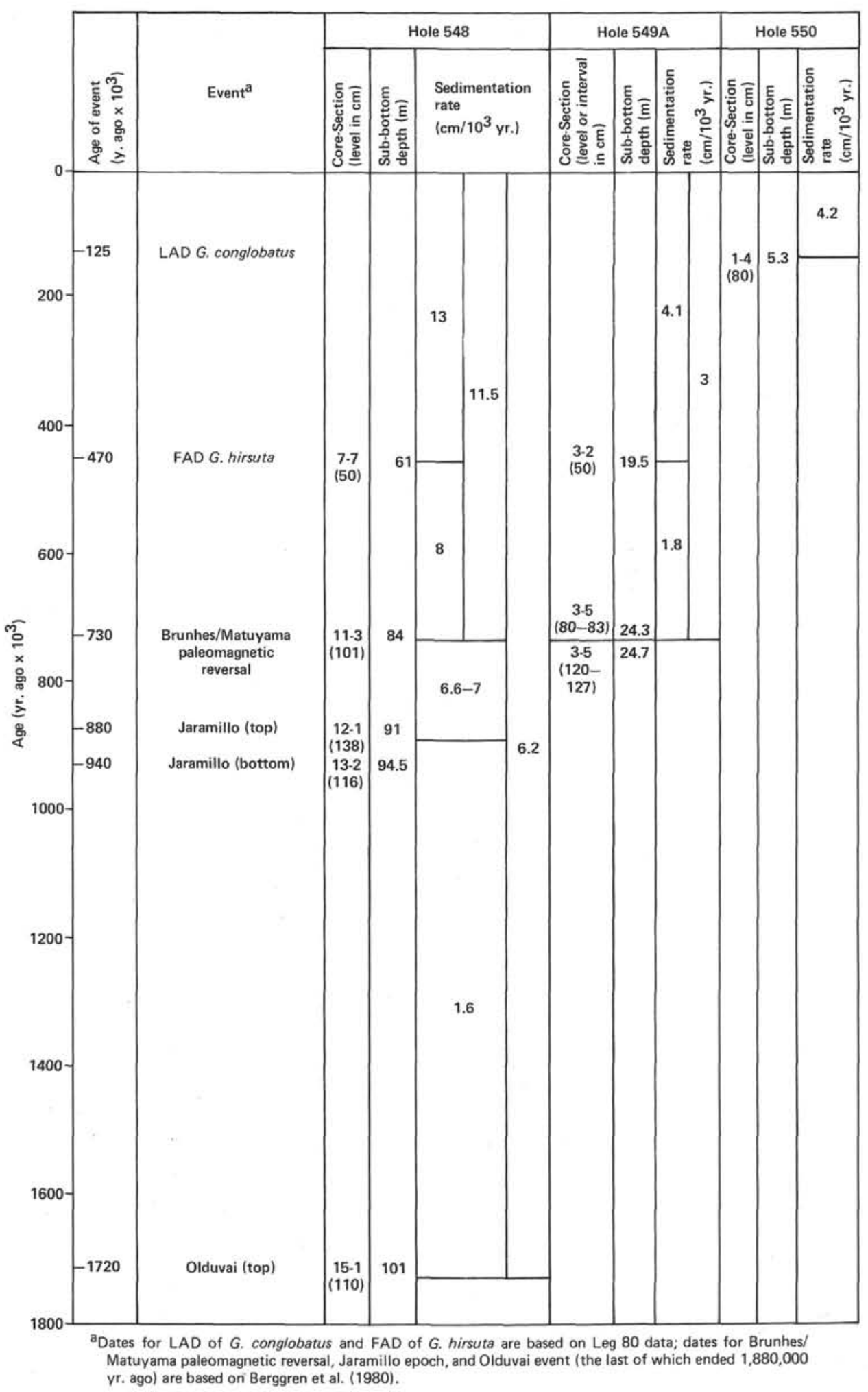

Figure 7. Estimated average sedimentation rate of the different holes between some chronologic datums. 


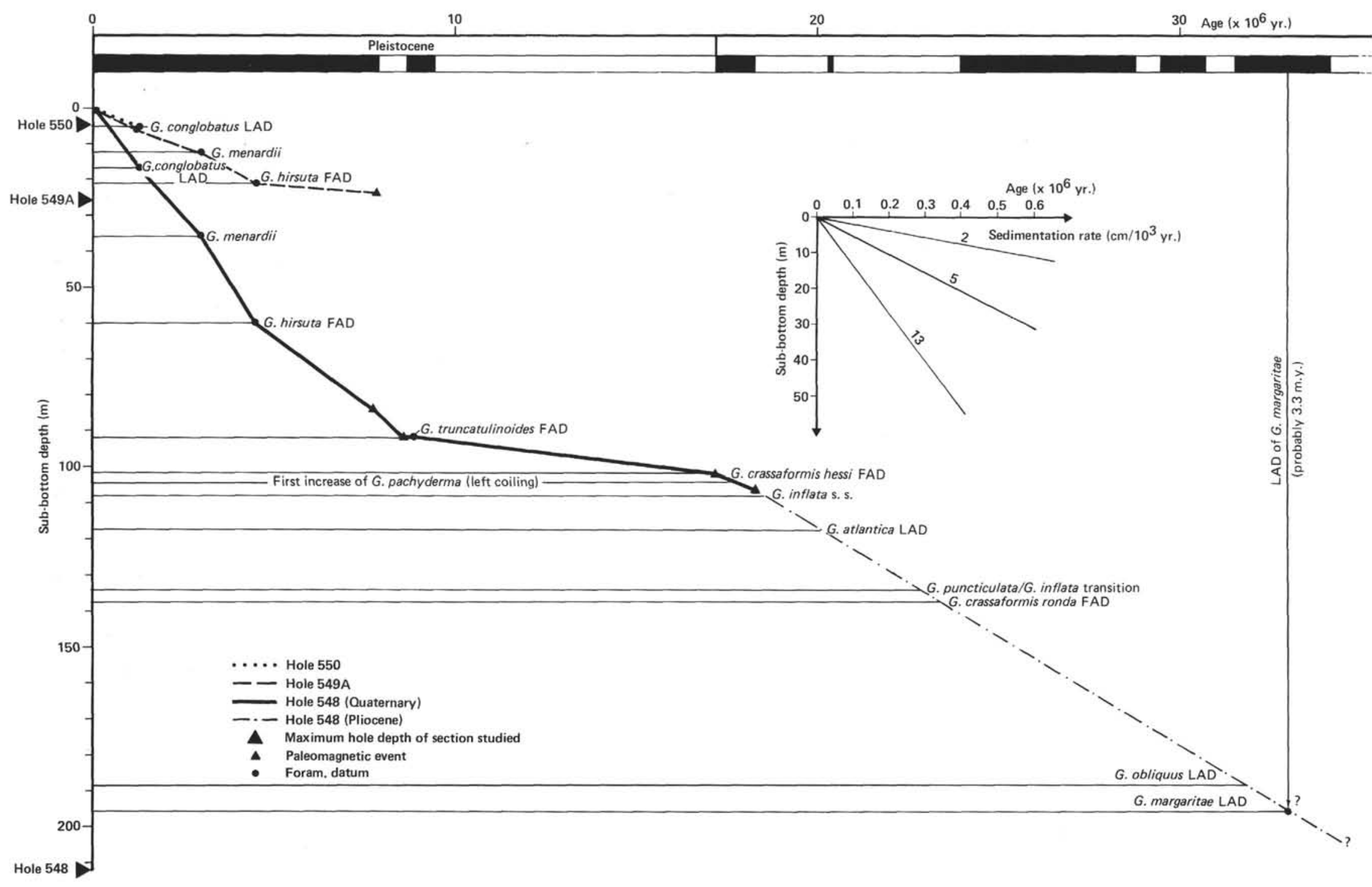

Figure 8. Estimate of average Pliocene-Quaternary sedimentation rates (Holes 548, 549A, and 550), with some planktonic foraminifer events. 
istics and gauge the development of the Quaternary microfauna. Thus, the extinctions and appearances of the microfaunal species most often used to define zones and subzones are difficult to locate (date) with any accuracy. The disappearance of Globigerina atlantica clearly takes place in the upper Pliocene (below the Quaternary base). Its extinction cannot mark this base (Poore and Berggren, 1975; Berggren, 1972). Compared with the study by Poore and Berggren (1975), the interval between the FAD of Globorotalia crassaformis and the disappearance of G. margaritae (Fig. 3D) is too long (G. crassaformis appears too late). However, it is also true that it is difficult to date the disappearance of $G$. margaritae; the age proposed by Poore and Berggren of $3.3 \mathrm{~m}$.y. appears rather old for this region, because it suggests a relatively low sedimentation rate, which seems to contradict the sedimentation rates for the underlying sediments. Estimates of chronological age should become more accurate as knowledge of the paleomagnetic scale improves.

\section{PALEOCLIMATOLOGY}

The recovery of a continuous Quaternary section permitted the paleoclimatology of these northern regions to be studied. The approach we adopted to reconstruct the climates was to assess the variations in physico-chemical parameters as revealed through the study of planktonic foraminifers.

\section{Methodology}

The method used was that developed by Imbrie and Kipp (1971) for the quantitative analysis of micropaleontological data. This method allows the quantitative estimation of the oceanographic variables that prevailed in the past. It is made up of three programs: CABFAC, REGRESS, and THREAD. The CABFAC program treats recent data (calculates percentages, performs standardization) and determines the chief components (performs a Q-mode factor analysis). In the REGRESS program, multiple regression analysis is used to define the relationships among factors and oceanographic variables. The regression analysis yields equations that are used in the THREAD program to estimate these oceanographic variables from factor loadings in samples for which these parameters are unknown.

\section{Data and Material}

Data are summarized in three matrices. The first covers the qualitative and quantitative analyses of recent thanatocoenosis and consists of regression equations. The samples used are those studied by Kipp (1976), Kel$\log$ (1973), Pujol (1980), and Duprat (1983). The matrix employed consists of 280 surface samples distributed throughout the North Atlantic from the Norwegian Sea to the Azores. The transfer function employed (FT 280) is that worked out by Duprat (1983).

The second matrix consists of the physico-chemical variables that correspond to the sampling locations for the first matrix. The summer and winter temperatures used were those established by the U.S. Naval Oceanographic Office (1958 and 1967).
The third matrix corresponds to faunal data for the sites investigated in this study. We used Cores 1 to 6 from Hole 549A and Cores 7 to 16 from Hole 548 for the biostratigraphic analysis. The matrix consists of 30 species and 146 samples.

\section{Analytical Process}

The CABFAC program distinguished six factors or assemblages. Each assemblage is defined according to its faunal content (Appendixes A and B). Their variance was as follows: $36.3 \%$ subarctic assemblage, $28.6 \%$ tropical assemblage, $16.2 \%$ arctic assemblage, $6.3 \%$ summer tropical assemblage, $4.9 \%$ winter tropical assemblage, and $2.3 \%$ gyre margin.

The distribution of these assemblages is approximately the same as described by Kipp (1976), Pujol (1980), and Thiede (1977); it corresponds to the pattern found by Duprat (1983).

The THREAD program, which dealt with the older levels of Holes 548 and 549A, responded fairly well at all the levels studied. The commonality observed was more than 0.90 at Hole 549A (Appendix B). Certain samples from Hole 548 showed commonalities of about 0.85 . The commonalities for Hole 548 , Core 8 , Section 2, 90-91 cm; Core 8, Section 2, 140-147 cm; and Core 8 , Section $3,69-70 \mathrm{~cm}$ are respectively $0.71,0.57$, and 0.77 .

\section{Estimated Temperatures}

The estimation of paleotemperatures for the Quaternary sequence has been the subject of numerous previous surveys, especially those performed by the CLIMAP Program. The first attempt to develop temperature estimates focused on 18,000 yr. ago, a reference age for conditions of maximum glaciation (CLIMAP Project Members, 1976).

Several studies in the North Atlantic went beyond this reference age to attempt to improve our understanding of glacial/interglacial changes. Recent data on paleotemperature estimates involve areas close to the investigation zone, that is, the eastern part of the North Atlantic gyre (Crowley, 1976); Ireland (Sancetta, Imbrie, Kipp, et al., 1972; Sancetta, Imbrie, and Kipp, 1973); the northeast Atlantic (Ruddiman and Glover, 1975; Ruddiman and McIntyre, 1976; McIntyre et al., 1976; Pujol, 1980); the Norwegian Sea (Kellog, 1977); and the Euroafrican margin and the Bay of Biscay (MolinaCruz and Thiede, 1978; Pujol, 1980). However, these analyses usually concern only the last glacial cycle (0$127,000 \mathrm{yr}$. ago). The paleoclimatic analysis of the entire Quaternary sequence has rarely been studied in tropical zones (Briskin and Berggren, 1975). This is, therefore, the first time that such an investigation has taken place in the middle latitudes, where climatic variations due to the movement of glaciers are significant (Ruddiman and McIntyre, 1976, 1977, and 1981).

Our estimates of the fluctuations through time of the summer and winter temperatures of surface water are given in Appendixes C and D and are summarized in Figures 9 and 10, which show the whole Quaternary sequence and the Pliocene/Quaternary transition. 
In Hole 549A, the estimates of present-day summer and winter surface water temperatures derived from a sample at the surface $\left(1-4 \mathrm{~cm} \mathrm{~m}\right.$ sub-bottom) are $16.9^{\circ} \mathrm{C}$ and $10.5^{\circ} \mathrm{C}$. These temperatures correspond to the mean values given in the Atlas.

During periods of maximum glaciation (Isotopic Stage $2,18,000$ yr. ago), surface water temperatures are estimated to have averaged approximately $5.8^{\circ} \mathrm{C}$ in summer and $0^{\circ} \mathrm{C}$ in winter. These estimates correspond both to those found by the CLIMAP Project (CLIMAP Project Members, 1976) and to those found in more detailed studies of the North Atlantic by Pujol (1980) and Duprat (1983). There is a difference of about $10^{\circ} \mathrm{C}$ between the present and glacial periods that is characteristic of the changes in geographic regions that are subject to intermittent glaciation. The lower summer and winter temperatures can be recognized in Subdivisions II, VI, XII, and XX in Hole 549A and in Subdivison XXVI in Hole 548. The warmer temperatures were observed in Subdivisions V, VII, IX, XIII, and XV at Hole 549A and XVII, XIX, XXI, and XXIX in Hole 548 (Figs. 9 and 10). The difference between summer and winter temperatures within a single climatic period is on the order of $6^{\circ} \mathrm{C}\left(4.5^{\circ} \mathrm{C}\right.$ to $\left.8^{\circ} \mathrm{C}\right)$ and corresponds to present-day seasonal fluctuations.

The general pattern of temperature estimates shows that a slight general cooling takes place from Subdivision XIII to the present. In fact, a climatic deterioration occurred in the Quaternary sequence at Subdivision XIII, approximately $470,000 \mathrm{yr}$. ago.

\section{CONCLUSIONS}

The analysis of Quaternary planktonic microfauna acquired during Leg 80 from Holes 548, 549A, and 550 permitted various faunal assemblages or groupings of 24 species or varieties to be recognized. An investigation of the underlying Pliocene section revealed the presence of 11 of the most characteristic species. The biostratigraphic analysis covered 279 samples.

The biostratigraphy of the Quaternary sequence is based on the appearances and disappearances of specific taxa and on morphological variations (changes in coiling ratio) in several subtropical species. Bioclimatic differentiation of the sedimentary column in the Quaternary was made possible by observing faunal associations. The base of the Quaternary is located at the top of the Olduvai Event $(1,720,000$ yr. ago).

Three zones were recognized in the Pliocene. The upper zone (the Globoratalia inflata Zone) covers the Pliocene/Quaternary transition. The Quaternary ( $G$. truncatulinoides taxonomic range Zone) is subdivided into three subzones: G. truncatulinoides, G. hirsuta, and Turborotalia humilis.

This biozonation applies to the northern part of the North Atlantic, which was subjected during the Quaternary to fluctuating climatic conditions and differs from conditions in tropical regions. Therefore a correlation of biozonations in these two domains does not yield satisfactory results. Climatic variations governed the microfaunal distribution, and about 30 bioclimatic subdivisions could be distinguished. A number of these subdivisions are comparable to the isotopic stages traditionally recognized in the Quaternary. The interpretation of these observations with respect to the paleomagnetic scale permits a chronological assessment to be made of the most important events.

The Pliocene/Quaternary boundary does not appear to be well marked in the planktonic foraminiferal assemblages. The first indication of cooling appears at the base of the Olduvai Event. The appearance of G. truncatulinoides, which is usually considered a marker of the Quaternary base, occurs at the Jaramillo Event.

The first appearance of $G$. hirsuta during Subdivision XIII accompanies an important lithologic and faunal change.

The distribution of most characteristic Pliocene taxa (such as G. margaritae and Globigerina atlantica) cannot be dated easily because of a lack of accurate paleomagnetic data.

Surficial Quaternary paleoenvironments in the North Atlantic were studied. Some 146 samples from Holes $549 \mathrm{~A}$ and 548 yielded estimates of summer and winter surface water temperatures. Curves of these estimates corroborate the climatic subdivisions perceived on the basis of an examination of the microfauna.

During glacial stages the average summer water temperature was approximately $5.8^{\circ} \mathrm{C}$; the average winter water temperature was approximately $0^{\circ} \mathrm{C}$. For both seasons, the difference between the present and the glacial period is about $10^{\circ} \mathrm{C}$. Within a given period (glacial or warm stage), the difference between summer and winter estimates is on the order of $6^{\circ} \mathrm{C}$. Two climatic periods are distinguished in the Quaternary sequence; a change appears to occur approximately $470,000 \mathrm{yr}$. ago.

\section{TAXONOMIC NOTES}

This nonsystematic list consists only of the species and forms from the Goban Spur that are illustrated in the plates. The list of species and forms follows the order of the illustrations. For the Quaternary species, this order takes into account the species' present biogeographic distribution in the North Atlantic Ocean. The Pliocene species appear in alphabetical order after the Quaternary species. The specimens illustrated have been deposited in the paleotological collection of the Institut de Géologie du Bassin d'Aquitaine of the University of Bordeaux I (France).

\section{Quaternary Fauna}

Globigerina pachyderma (Ehrenberg) left-coiling (Pl. 1, Figs. 1-4). This is the only species from the Arctic assemblage. Because it lives in cold water, the frequency of its occurence is suggestive of the temperature of the water.

Globigerina pachyderma (Ehrenberg) right-coiling (PI. 1, Figs. 5-9). This species is abundant throughout the Quaternary of the northeast Atlantic. Its occurrence reaches a climax at the base of the Globorotalia truncatulinoides Zone.

Globigerinita uvula (Ehrenberg) (Pl. 1, Fig. 10). Very scarce in the coarse fraction.

Globigerina quinqueloba Natland (Pl. 1, Figs. 11-14).

Globigerina bulloides d'Orbigny (Pl. 2, Figs. 1-4).

Globigerina cariacoensis Rögl and Bolli (Pl. 2, Figs. 5-12). This species appears to be part of the variability interval of $G$. bulloides. It differs, however, in its large size, higher spiral coiling, and greater number of chambers in the last whorl. This species probably reflects the ecological conditions special to the Caribbean region.

Globigerina bulloides d'Orbigny var. (PI. 3, Figs. 1-6). This form, which is assumed to belong to the $G$. bulloides species, differs by a less lobate outline and a low arched aperture. It seems to disappear 


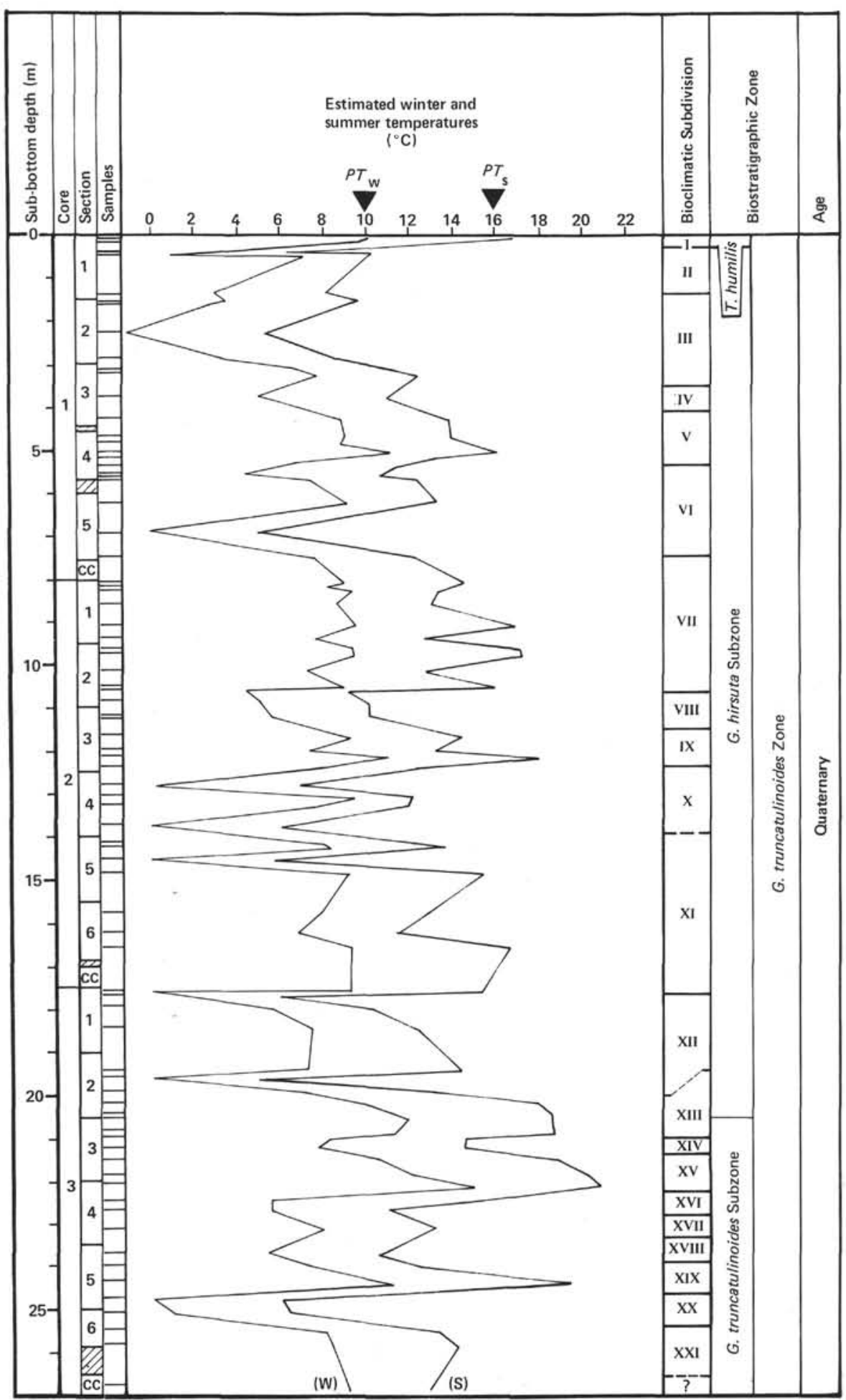

Figure 9. Estimated surficial temperature at Hole 549A (Cores 1-3) during winter (W) and summer (S) and comparison with biostratigraphic and bioclimatic subdivisons. $P T_{\mathrm{w}}=$ present winter temperature, $P T_{\mathrm{s}}=$ present summer temperature.

in the late Pliocene. Nevertheless, its characteristics lead us to illustrate this form near the recent subarctic species.

Globigerinita glutinata (Egger) (Pl. 3, Figs. 7-9).

Globorotalia scitula (Brady) form 1 (Pl. 3, Figs. 10, 13; Pl. 4, Figs. 3-6).

Globorotalia bermudezi (Rögl and Bolli) (Pl. 3, Figs. 11, 12). This species differs from the $G$. scitula form 1 mainly by its flat distal face and straight intercameral sutures. Inasmuch as its distribution resembles that of $G$. scitula form 1, both forms were analyzed under the name of the first for purposes of analysis.

Globorotalia scitula (Brady) form 2 (Pl. 3, Fig. 14; Pl. 4, Figs. 1-2).

This form corresponds well to the species description. It differs, however, by its size and its coarse perforated test. Its stratigraphic range distribution is also appreciably different; it was observed chiefly in the early Quaternary (Matuyama Epoch and base of Brunhes Epoch). 


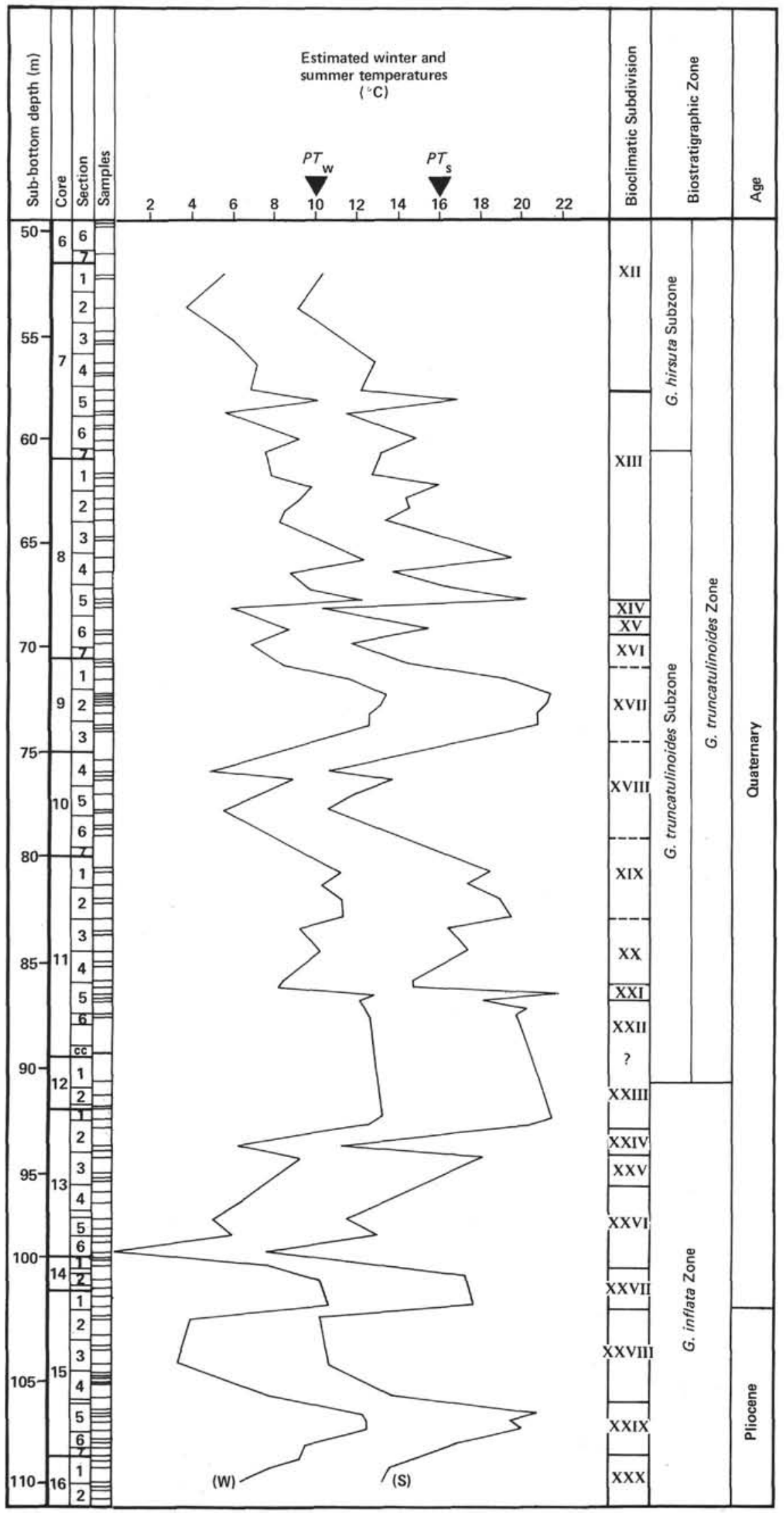

Figure 10. Estimated surficial temperature at Hole 548 (Cores 7-16) during winter (W) and summer (S) and comparison with biostratigraphic and bioclimatic subdivisions. $P T_{\mathrm{w}}=$ present winter temperature, $P T_{\mathrm{s}}=$ present summer temperature. 
Orbulina universa d'Orbigny (Pl. 4, Fig. 7).

Hastigerina siphonifera (d'Orbigny) (Pl. 4, Figs. 8-10).

Globorotalia inflata (d'Orbigny) (Pl. 5, Figs. 1-2, 7-8, and 9-10). Figures 7 and 8 show transitional morphs of the G. puncticulata species. The forms illustrated in Figures 9 and 10 were defined as $G$. inflata aff; they are rare small-sized specimens that may be considered to be within the species variability.

Globorotalia inflata (d'Orbigny) var. (Pl. 5, Figs. 3-6). The distribution of this form is very limited on the stratigraphic scale. It was distinguished only in bioclimatic Subdivision XIII in Holes 548 and 549A. It differs from the characteristic form by a higher coil and a porcelaneous shell.

Globorotalia truncatulinoides (d'Orbigny) (Pl. 5, Figs. 11-14). This species is characteristic of Quaternary section and occurs at Goban Spur for the first time in the deposits of the Jaramillo Event.

Globorotalia hirsuta (d'Orbigny) (Pl. 6, Figs. 1-12). Morphological variations of this species have been described at length in a study by Pujol (1975) of the Bay of Biscay sediments

Turborotalita humilis (Brady) (Pl. 7, Figs. 1-3). This small species, characteristic of postglacial deposits, is almost nonexistent in the coarse fraction larger than $150 \mu \mathrm{m}$. Recognizing this species requires close observation of the fraction between 150 and $63 \mu \mathrm{m}$.

Globigerina calida Parker (Pl. 7, Fig. 4).

Globigerinoides ruber d'Orbigny (Pl. 7, Fig. 5).

Globorotalia crassaformis crassaformis (Galloway and Wissler) (Pl. 7, Figs. 6-8; Pl. 9, Figs. 4-6). Recent forms are largely different from the Pliocene forms (Pl. 9, Fig. 4-6). The profile of the latter is more angular. This species is still very badly differentiated within the Pliocene.

Globorotalia crassaformis hessi (Bolli and Premoli Silva) (Pl. 7, Figs. 9-11). This species occurs as early as at the base of the Quaternary. It is part of the $G$. crassaformis species distribution in Hole 549A.

Globorotalia crassaformis oceanica Cushman and Bermudez (Pl. 8, Figs. 1-5). This species is quite scarce. It is found in the range of diversification of the $G$. crassaformis species.

Globorotalia crassaformis ronda Blow (Pl. 8, Figs. 6-14; Pl. 9, Figs. 1-3). This subspecies appears in the Pliocene section and does not seem to develop after about $470,000 \mathrm{yr}$. ago.

Globorotalia crassula Cushman and Stewart (P1. 9, Figs. 7-10; P1. 10, Figs. 1-7). The Berggren and Amdurer (1973) description is employed for the Pliocene-Pleistocene deposits of the Rio Grande Rise (Berggren, 1977; Pujol and Duprat, 1984). This species occurs in the Pliocene but also at the lowest extremity of the Quaternary. At its appearance, the population is dominantly dextral, but it very quickly becomes sinistral. It is easy to differentiate this species from G. margaritae and G. hirsuta, with which it shares the same morphological characteristics, by the absence of a pronounced carina.

Globorotalia tumida (Brady) (Pl. 10, Fig. 8). This species has been grouped with $G$. menardii.

Globorotalia menardii (d'Orbigny) (Pl. 10, Fig. 9).

Globorotalia cf. flexuosa (Koch) (Pl. 10. Fig 10). Quite infrequent, this form is associated with the $G$. menardii group.

Globigerinoides conglobatus (Brady) (Pl. 10, Fig. 11). The disappearance of this species in the Goban Spur area takes place at the base of the last interglacial stage (Isotopic Stage 5).

\section{Pliocene Fauna}

Globigerina atlantica (Berggren) (P1. 11, Figs. 1-9). This species disappears from the Goban Spur deposits before the first contemporary cooling of the Olduvai Event. Its last specimens show a decrease in the number of chambers of the last whorl (PI. 11, Fig. 1).

Globorotalia puncticulata (Deshayes) (PI. 12, Figs. 1-8). It is assumed that a phylogenic transition of this species with $G$. inflata takes place in the late Pliocene.

Globorotalia margaritae (Bolli and Bermudez) (PI. 12, Figs. 9-11). This species characterizes the early Pliocene.

Globigerinoides obliquus (Bolli) (PI. 12, Fig. 12).

Sphaerodinellopsis subdehiscens (Blow) (Pl. 12, Fig. 13).

\section{ACKNOWLEDGMENTS}

The writers wish to thank the Deep Sea Drilling Project for having made Quaternary samples from Leg 80 available for the present study. The Institut de Géologie du Bassin d'Aquitaine and the Laboratory of
Oceanography (L.A. 197) of the University of Bordeaux I provided laboratory facilities and technical help. The authors wish to express their thanks to J. Sigal for making available the material of the Pliocene of Site 548. M. Caralp. J. P. Peypouquet, H. Townsend, and C. Vergnaud Grazzini have contributed to this study through discussions. We are also very grateful to W. A. Berggren, J. C. Duplessy, P. C. de Graciansky, and W. Ruddiman, who reviewed the manuscript.

The scanning electron micrographs were taken by M. Leblanc of the Centre de Microscopie Électronique of the University of Bordeaux I.

This investigation was supported in part by I.G.B.A. and in part by a grant from A.T.P. Geology and Geophysics of the Ocean (C.N.R.S., France).

\section{REFERENCES}

Backman, J., 1979. Pliocene biostratigraphy of DSDP Sites 111 and 116 from the North Atlantic Ocean and the age of Northern Hemisphere glaciation. Stockholm Contrib. Geol., 32:115-137.

Bé, A. W. H., 1977. An ecological, zoogeographic and taxonomic review of Recent planktonic Foraminifera. In Ramsay, A. T. S. (Ed.), Oceanic Micropaleontology (Vol.1): London (Academic Press), pp. 1-100.

Berggren, W. A., 1972. Cenozoic biostratigraphy and paleobiogeography of the North Atlantic. In Laughton, A. S., Berggren W. A. et al., Init. Repts. DSDP, 12: Washington (U.S. Govt. Printing Office), $965-1000$.

1977. Late Neogene planktonic foraminiferal biostratigraphy of the Rio Grande Rise (South Atlantic). Mar. Micropaleontol., 2:265-313.

Berggren, W. A., and Amdurer, M., 1973. Late Paleogene (Oligocene) and Neogene planktonic foraminiferal biostratigraphy of the Atlantic Ocean (lat. $30^{\circ} \mathrm{N}$ to lat. $30^{\circ} \mathrm{S}$ ). Riv. Ital. Paleontol., 79: 337-392.

Berggren, W. A., Burckle, L. H., Cita, M. B., Cooke, H. B. S., Funnell, B. M., Gartner, S., Hays, J. D., Kennett, J. P., Opdyke, N. D., Pastouret, L., Shackleton, N. J., and Takayanagi, Y., 1980. Towards a Quaternary time scale. Quat. Res., 13:277-302.

Blanc, P. L., 1981. Paleoclimatologie isotopique et histoire de l'eau profonde atlantique depuis 15 millions d'années. Univ. Paris, Thesis No. 2474.

Blow, W. H., 1969. Late middle Eocene to Recent planktonic foraminiferal biostratigraphy. In Brönnimann, P., and Renz, H. H. (Eds.), Proc. First Internat. Conf. Planktonic Microfossils: Leiden (E. J. Brill), pp. 199-422.

Bolli, H. M., and Premoli Silva, I., 1973. Oligocene to Recent planktonic Foraminifera and stratigraphy of the Leg 15 sites in the Caribbean Sea. In Edgar, T. N., Saunders, J. B., et al., Init. Repts. DSDP, 15: Washington (U.S. Govt. Printing Office), 475-497.

Briskin, M., and Berggren, W. A., 1975. Pleistocene stratigraphy and quantitative paleooceanography of tropical North Atlantic Core V16-205. In Saito, T., and Burckle, L. (Eds.), Late Neogene Epoch Boundaries: New York (Micropaleontology Press), pp. 167-198.

Caralp M., 1971. Les foraminifères planctoniques du Pléistocène terminal dans le Golfe de Gascogne. Interprétation biostratigraphique et paléoclimatique. Bull. Inst. Geol. Bassin Aquitaine, 11: $1-191$.

Caralp, M., Duprat, J., Moyes, J., and Pujol, C., 1974. La stratigraphie du Pléistocène supérieur et de l'Holocène dans le Golfe de Gascogne. Essai de synthèse des critères actuellement utilisables. Boreas, 3:35-40.

CLIMAP Project Members, 1976. The surface of the ice-age earth. Science, 191(4232):1131-1137.

Crowley, T., 1976. Fluctuations of the eastern North Atlantic gyre during the last 150,000 yr. [Ph. D. dissert.]. Brown Univ., Providence, R. I.

Duprat, J., 1983. Contribution à l'analyse biostratigraphique et des environnements du Quaternaire terminal dans l'Atlantique nordest ibérique. Bull. Inst. Geol. Bassin Aquitaine, 33:71-150.

Ericson, D. B., and Wollin, G., 1956. Micropaleontological and isotopic determinations of Pleistocene climates. Micropaleontology, 2:257-270.

1968. Pleistocene climates and chronology in deep sea sediments. Science, 1621227-1234. 
Imbrie, J., and Kipp, N. G., 1971. A new micropaleontological method for quantitative paleoclimatology: application to a late Pleistocene Caribbean core. In Turekian, K. K. (Ed.), The Late Cenozoic Glacial Ages: New Haven (Yale Univ. Press), pp. 71-181.

Kellogg, T. B., 1973. Late Pleistocene climatic record in Norwegian and Greenland sea deep sea cores [Ph.D. dissert.]. Columbia Univ., N.Y.

1977. Paleoclimatology and paleo-oceanography of the Norwegian and Greenland seas. The last 450,000 years. Mar. Micropaleontol., 2:235-249.

Kipp, N. G., 1976. New transfer function for estimating past sea surface conditions from sea bed distribution of planktonic foraminiferal assemblages in the North Atlantic. Geol. Soc. Am. Bull., 145: 3-41.

McIntyre, A., Kipp, N. G., Bé, A. W. H., Crowley, T., Kellogg, T., Gardner, J. V., Prell, W., and Ruddiman, W. F., 1976. Glacial North Atlantic 18,000 years ago. A climap reconstruction. Geol. Soc. Am. Bull., 145:43-76.

Molina-Cruz, A., and Thiede, J., 1978. The glacial eastern boundary current along the Atlantic Eurafrican continental margin. Deep Sea Res., 25:337-356.

Poore, R. Z., and Berggren, W. A., 1975. Late Cenozoic planktonic foraminiferal biostratigraphy and paleoclimatology of Hatton Rockall Basin: DSDP Site 116. J. Foram. Res., 5:270-293.

Pujol, C., 1975. Intérêt des variations du sens d'enroulement de $G$. truncatulinoides et de $G$. hirsuta (Pleistocène supérieur et Holocène du Golfe de Gascogne). Rev. Esp. Micropaleontol., Num. spec., pp. 107-116.

1980. Les foraminifères planctoniques de l'Atlantique nord au Quaternaire. Ecologie-Stratigraphie-Environnement. Mem. Inst. Geol. Bassin Aquitaine, No. 10.

Pujol, C., and Caralp M., 1974. Variations du sens d'enroulement des foraminifères planctoniques dans l'interprétation stratigraphique du Quaternaire terminal de l'océan Atlantique nord. Bull. Inst. Geol. Bassin Aquitaine, 16:31-50.

Pujol, C., and Duprat, J., 1977. Intérêt de Globorotalia hirsuta dans le Quaternaire de l'Atlantique nord-oriental [paper presented at 5 th annual meeting, Réun. Annu. Sci. Terre, Rennes, France, 19-22 April].

1984. Quaternary planktonic foraminifers of the southwestern Atlantic (Rio Grande Rise) Deep Sea Drilling Project Leg
72. In Baker P., Johnson, D., et al., Init. Repts. DSDP, 72: Washington (U.S. Govt. Printing Office), 601-622.

Pujol, C., Duprat, J., Gonthier, E., Peypouquet, J. P., and Pujos-Lamy, A., 1974. Résultats préliminaires de l'étude effectuée par l'institut de géologie du Bassin d'Aquitaine concernant la mission FAEGAS (25 Juin-17 Juillet 1973) dans l'Atlantique nord-est. Bull. Inst. Geol. Bassin Aquitaine, 16:65-94.

Ruddiman, W. F., and Glover, L. K., 1975. Subpolar North Atlantic circulation at 9,300 years B.P. Faunal evidence. Quat. Res., 5:361-389.

Ruddiman, W. F., and McIntyre, A., 1976. Northest Atlantic paleoclimatic change over the past 600,000 years. Mem. Geol. Soc. Am., 145:111-146.

1977. Late Quaternary surface ocean kinematics and climatic change in the high-latitude North Atlantic. J. Geophys. Res., 82:3877-3887.

1981. The North Atlantic Ocean during the last deglaciation. Paleogeogr. Paleoclimatol. Paleoecol., 35:145-214.

Sancetta, C., Imbrie, J., and Kipp, N. G., 1973. Climatic record of the past 130,000 years in North Atlantic deep sea Core V23-83. Correlation with the terrestrial record. Quat. Res., 3:110-116.

Sancetta, C., Imbrie, J., Kipp, G., McIntyre, A., and Ruddiman, W. F., 1972. Climatic record in north Atlantic deep sea core V23-82: comparison of the last and present interglacials based on quantitative time series. Quat. Res., 2:363-367.

Thiede, J., 1977. Aspects of the variability of the glacial and interglacial North Atlantic eastern boundary current (last 150,000 years). Meteor Forschungsergeb. Reihe $C$., 28:1-36.

Thierstein, H. R., Geitzenauer, K., Molfino, B., and Shackleton, N. J., 1977. Global synchroneity of late Quaternary coccolith datums: validation by oxygen isotopes. Geology, 5:400-404.

U.S. Naval Oceanographic Office, 1958. Oceanographic Atlas of the Polar Seas. Part II: Arctic. H.O. Pub. No. 705: Washington (U.S. Govt. Printing Office).

1967. Oceanographic Atlas of the North Atlantic Ocean. Section II: Physical Properties. Pub. No. 700: Washington (U.S. Govt. Printing Office).

Date of Initial Receipt: April 12,1983

Date of Acceptance: July 8, 1983 
APPENDIX A

Varimax Factor Matrix from Analysis of Hole 548 (Cores 7-16)

\begin{tabular}{|c|c|c|c|c|c|c|c|c|}
\hline \multirow[b]{2}{*}{ Sample } & \multirow{2}{*}{$\begin{array}{l}\text { Core-Section } \\
\text { (depth in cm) }\end{array}$} & \multirow[b]{2}{*}{ Commonality } & \multicolumn{6}{|c|}{ Factor assemblages } \\
\hline & & & 1 & 2 & 3 & 4 & 5 & 6 \\
\hline 1 & 7.1 .76 .75 &.$\varsigma 551$ & .2351 &. $\operatorname{col} \varepsilon$ & .5533 & .0631 & -.1603 & -.0014 \\
\hline 2 & 7.6 .84 .85 & $.5 s \leq 0$ & .1264 & -.COJ\& & .9770 & .0582 & -.1451 &. .0015 \\
\hline 3 & $7.7,104.111$ & .9464 & .8626 & .0077 & .2333 & .0205 & $-.3 c 08$ & .0132 \\
\hline 4 & $7.4,40.43$ & $.8 C 56$ & .5903 & $.<1 \geq 1$ & .6507 & .0337 & $-.1 S C 9$ & -.0005 \\
\hline 5 & $7.2,103.117$ & .5481 & .8848 &.$<1 t^{2}$ & .2757 & $.040 \mathrm{C}$ & -.1450 & .0095 \\
\hline$t$ & $7.5,19.22$ & $.98>4$ & .7702 & $.01 C^{7}$ & .5653 & $.047 \mathrm{C}$ & -.2611 & .0114 \\
\hline 7 & $7.5 .111 .11 t$ & . ક ع11 & .9097 & .0361 & .1212 & .2654 & -.2534 & .0529 \\
\hline e & $7.5 .14 C .141$ & .5513 & .7043 &. $\operatorname{coc} 1$ & .5959 & .6755 & $-.30<7$ & .0202 \\
\hline 9 & $7 . t, 11[.117$ & $.25 t 2$ & .9025 & $.071 t$ & .0763 & - C 26C &. .1789 & -.0045 \\
\hline 10 & $7,7,10,13$ & .5158 & .9426 & $.025 ?$ & .1065 &.$- C \Leftrightarrow 9 C$ & $-.11 \varsigma 1$ & -.0111 \\
\hline 11 & $8.1, \mathrm{tg}, 7 \mathrm{C}$ & $.5 C S E$ & $.94 C 0$ & $.022 t$ & .0694 & -.0749 & $-.1 i z 4$ & -.0101 \\
\hline 12 & $8.1,123.13 \mathrm{C}$ & .9324 & .9548 & .0547 & .0666 & $. C 4 \geq 5$ & $-.1 C \in C$ & $.012 J$ \\
\hline 13 & $8 . i, 40.47$ & .9849 & .8632 & $.05 C 6$ & .0552 &.$C 80 C$ & -.3574 & .6109 \\
\hline 14 & $\varepsilon .6,9 C .91$ & .7157 & .7751 & $.04 i c$ & $.10 € 1$ & $.1 \% 9 \mathrm{C}$ & $-.28 C 2$ & .0221 \\
\hline 15 & $8 . i, 14 \mathrm{C}, 147$ & .2776 & .6738 & .0612 & .0971 & .0446 & -.2252 & -.0090 \\
\hline 16 & $8.2, \in 5.70$ & .7752 & .8092 & .0687 & .6857 & $.237 t$ & -.2345 & .0303 \\
\hline 17 & $8.4, \overline{c C} \cdot \overline{-27}$ & .9723 & .9443 & $.046 t$ &.$[319$ & .6545 & -.0974 & .0565 \\
\hline $\begin{array}{l}18 \\
19\end{array}$ & 8.2 .90 .91 & $\begin{array}{r}-9429 \\
58=7\end{array}$ & .8576 & -0412 & .2368 & .1849 & $-.2 \geq 55$ & .0284 \\
\hline $\begin{array}{l}19 \\
20\end{array}$ & $\begin{array}{l}8.5,60.27 \\
\varepsilon .5, \in 5.7 \mathrm{C}\end{array}$ & $\begin{array}{l}-5,5<7 \\
.5<71\end{array}$ & $\begin{array}{l}.9886 \\
.9325\end{array}$ & $\begin{array}{l}.0442 \\
.0416\end{array}$ & $\begin{array}{l}-c 560 \\
-1305\end{array}$ & $\begin{array}{l}.0453 \\
.2872\end{array}$ & $\begin{array}{c}-C \\
0\end{array} 345$ & $\begin{array}{l}.0057 \\
.0589\end{array}$ \\
\hline ¿1 & $8.5,1 C z=110$ & .9858 & .2629 & $.01 C 2$ & .9339 & $.1<43$ & $\begin{array}{r}.0585 \\
-.1767\end{array}$ & $.0 C 86$ \\
\hline 22 & $8 . t .69 .7 \mathrm{C}$ & .9736 & .8918 & .0278 & .2301 & .2215 & -.1045 & .0698 \\
\hline 23 & $2 \cdot t, 1=4,12=$ & $.9 \leq 24$ & .5098 & .0248 & .7848 & $. \quad \leq 510$ & $-.14: 6$ & .0406 \\
\hline 24 & $9.1,20.23$ & .9114 & .8350 & $.034 C$ &.$? 906$ & .2275 & -.1242 & .0410 \\
\hline 25 & $5.1,1 \mathrm{CC} .107$ & .5573 & .9344 & $.06 t i$ & .1063 & $. \hat{c} \leq 4 t$ &.$C \&[t$ & .0452 \\
\hline i6 & $9 . i, \overline{c 0} .23$ & $.0 \leq t 4$ & $.85 C 1$ & $.05 t 1$ & .0433 & .4677 & $-. C 1<C$ & .0990 \\
\hline 27 & $9.2,43.44$ & . $c \in 12$ & .8759 & $.07 \approx 9$ & .0473 & .4158 & $. c 8 \leq 1$ & .0792 \\
\hline 28 & $9.2,16 \bar{c}, 10 \mathrm{~S}$ & .0573 & .8435 & $.0>t 7$ & .0529 & .4745 & $-. c \leq \leq c$ & .0947 \\
\hline 29 & $5.2,20.23$ & $.5 E 57$ & .9049 & .0415 & .0315 & $.2 \leq 50$ & .1121 & .0746 \\
\hline 20 & $10.4,43.44$ & .9718 & $.5 C<0$ &.$C 2 C z$ & .7306 & .3866 & -.1751 & .0741 \\
\hline 31 & $10.4,114.116$ & . $5 \& 12$ & .7173 & $.055 C$ & .5384 & $.048 \times$ & $-. x \geq<2$ & .0355 \\
\hline 32 & $1 C .5,43.44$ & .9874 & .5294 & $.01 C s$ &.$\varepsilon 2 € 1$ & $.140 t$ & -.0268 & .0196 \\
\hline 23 & $1 C, 5,1 i C, 1 i z$ & .5574 & .2284 &.$- \cos 1$ & $.94 C 3$ & .0457 & -.0577 & .0021 \\
\hline 34 & $11.1,5<.53$ & $.97 \% 4$ & .9525 & $.10<1$ & $.18 C 4$ & $.141 t$ &.$- C 416$ & .0228 \\
\hline 35 & 11.1 .140 .147 & .5829 & .9832 & $.04 \geq 1$ & . 1377 & $-\cos 2$ & . CSE 1 & .0166 \\
\hline 36 & $11.2,50.53$ & $.5 t 36$ & .8894 & $-c \in t \bar{c}$ & .1972 & .3517 & .0272 & .0698 \\
\hline 37 & $11.2,14 \mathrm{C} .147$ &.$\varsigma Q C 6$ & .9477 & .0424 & .0272 & .1909 & .2278 & .0394 \\
\hline 38 & $11.3,50.53$ & $.98 t 2$ & .9818 & .0252 & .0227 &.$- c c J \varepsilon$ & .1451 & .0063 \\
\hline 39 & $11.3,139.12 \mathrm{t}$ & $.55 C 4$ & .9815 & $.02<8$ &.$<2 \in 3$ & . $c<42$ & $.14 \div 5$ & .0206 \\
\hline 40 & $11.4,140.147$ & .9779 & .9823 & $.015 C$ & - $4 \geq 05$ &.$-<5 \in 1$ &.$c s ? 3$ & $\because 0015$ \\
\hline 41 & $11.5,17.64$ & $.98 \mathrm{C3}$ & .9623 & $.024 C$ & .0227 & -.0718 & .2190 & -.0083 \\
\hline 42 & $11.5,5 C .53$ & .5553 & .8249 & $.05 \geq 2$ & .0100 & $.4 \in 15$ & $.2 \geq c 4$ & .0990 \\
\hline 43 & $11.5,92.97$ & $.54 \overline{<4}$ & .9410 & $.197 t$ & .0792 &.$- \operatorname{ccs} t$ & $-.1 C 77$ & -.0096 \\
\hline 44 & $11,5,143,150$ & $.5 \geq 49$ & .7479 & .0561 & .0654 & $.54 \geq C$ & $-.257 C$ & .1145 \\
\hline 45 & $11.6,8.15$ & .9912 & .9773 & .0577 &.$C 151$ & .1726 & .0295 & . .0439 \\
\hline 46 & $13.1,1 \geq 0.1 \geq 7$ &.$c \in \subseteq 5$ & .9055 & .0419 & .0162 &.$? 744$ & $-.01 \leq 5$ & .0843 \\
\hline 47 & $13.2,35.38$ & .5753 & .9075 & .0723 & .1072 & .3598 & $-. C<21$ & .0746 \\
\hline 48 & $12.2,120.121$ & .5963 & .3832 & .0135 & .9075 & $.1 \leq 21$ & - . C $1 \& 1$ & .0219 \\
\hline 49 & $. \bar{c} \bar{c} . \bar{c} q$ & $.9 C \& 4$ & $.7 \geq 12$ & $.04 \geq 2$ & .8075 & $.54 C 2$ & $-.14 \geq 5$ & .1277 \\
\hline so & $13.4,95.98$ & .9711 & .6397 & .0371 &.$t 115$ & .4229 & -.0169 & .0861 \\
\hline 51 & 13 & .5742 & .5415 & .0225 & .7197 & .2927 &.$- \operatorname{css} \theta$ & .0779 \\
\hline 52 & $13.5,130.132$ & .9426 & .5927 & $.04 C 9$ & .2547 & .4731 & $-.39 C 2$ & .0939 \\
\hline 53 & 0.27 .24 & .5659 & .3040 & .0157 & .8377 & .4108 & -.0218 & .0753 \\
\hline 56 & 47 & $.97 \in 1$ & .7120 & $.027 t$ & .5765 & .3261 & $-.1 \geq 12$ & .0631 \\
\hline 55 & $14.2,84.91$ & $.98 \div 5$ & $.9 \mathrm{CC6}$ & $.05 \div 7$ & .2026 & .8722 & $-.<1 \leq 2$ & .0517 \\
\hline 56 & $15.1,112.119$ & $.5 \in 25$ & .8547 & .0555 & .1918 & .3675 & -.2258 & .0761 \\
\hline 57 & .20 .33 & $.5 \leqslant 10$ & .4104 & .0254 & .7290 & .2906 & -.3158 & .0736 \\
\hline 58 & .110 .117 & $.94 C 8$ & .4576 & $.00 \geq 4$ & .7044 & . $\bar{T}$ Et & -.3981 & .0594 \\
\hline 59 & $15.4,110.117$ & $.98 \geq 5$ & .8004 &. $\cos \varepsilon$ & .5519 & $.1 \geq 18$ & -.0735 & .0293 \\
\hline$\in \mathrm{C}$ & $15.5, t^{2} \cdot t E$ & .5517 & .8853 & $.044 \bar{z}$ & . $C 414$ & .3679 & $.1 \leq ? 5$ & .0736 \\
\hline 61 & .52 .57 & $.5 \$ 10$ & $.96 \geq 1$ & $.021 t$ &. $\cos 3$ & $.15 \times C$ & $.15 ? 3$ & .0472 \\
\hline 62 & .130 .137 & .9715 & .9355 & . C1sC & . $\{212$ & .8962 & -. c sit & .0703 \\
\hline 63 &, $3 C_{0} 32$ & .5857 & $.98 \mathrm{C} 2$ & .0179 & .0259 & .0306 & .1567 & .0143 \\
\hline 64 & $15 . t, 12 z .12 t$ & .9737 & .9845 & $.01 \in 7$ & .0127 & $-.<4 \geq 1$ & .0357 & -.0003 \\
\hline 65 & $16.1,1[.11$ & $.5 \leq 50$ & $.96 \in 6$ & .0151 & .0257 & -.0157 & -.1352 & .0092 \\
\hline 66 & 16.1 .70 .77 & .9769 & .9822 & .0159 & .0262 & $-.102 C$ & $.02 \in 9$ & -.0139 \\
\hline 67 & $1.14 C .141$ & .9749 & .9750 & .0158 & .0606 & $-.100 t$ &. $\csc 4$ & -.0156 \\
\hline
\end{tabular}


APPENDIX B

Varimax Factor Matrix from Analysis of Hole 549A (Cores 1-3)

\begin{tabular}{|c|c|c|c|c|c|c|c|c|}
\hline \multirow[b]{2}{*}{ Sample } & \multirow{2}{*}{$\begin{array}{l}\text { Core-Section } \\
\text { (depth in } \mathrm{cm} \text { ) }\end{array}$} & \multirow[b]{2}{*}{ Commonality } & \multicolumn{6}{|c|}{ Factor assemblages } \\
\hline & & & 1 & 2 & 3 & 4 & 5 & 6 \\
\hline 1 & $1-1,1-4$ & .9937 & .9823 & .0614 & .0839 & .1563 & -.0801 & .0152 \\
\hline 2 & $1-1,8-11$ & .9883 & .9830 & .0752 & $.1 \cup 75$ & .0264 & -.0639 & -.0038 \\
\hline 3 & $1-1,39-42$ & .9812 & .3150 & .0123 & .9236 & .1210 & -.0698 & .0067 \\
\hline 4 & $1-1,42-45$ & .9848 & .0517 & -.0053 & .9894 & .0519 & -.0236 & -.0028 \\
\hline 5 & $1-1,137-14 C$ & .9024 & .1233 & -.0031 & .9842 & .0737 & -.0502 & .0008 \\
\hline 6 & $1-2,2-5$ & .9989 & .1068 & -.0342 & .9423 & .0313 & -.2889 & .0052 \\
\hline 7 & $1-2,5-8$ & .9949 & .0705 & -.0081 & .9753 & .0461 & -.1889 & -.0009 \\
\hline 8 & $1-2,70-77$ & .9831 & -.0091 & -.0121 & $.99 \cup 3$ & .0463 & -.0081 & -.0029 \\
\hline 9 & $1-2,130-137$ & .9948 & .0860 & -.0028 & .9877 & .0385 & -.1019 & -.0063 \\
\hline 10 & $1-3,10-13$ & .9958 & .4375 & .0297 & .7064 & .1309 & -.5265 & .0106 \\
\hline 11 & $1-3,2 c-23$ & .9591 & .7473 & .0547 & .4192 & .1743 & -.4372 & .0185 \\
\hline 12 & $1-3,70-77$ & .9385 & .3496 & .0155 & .7095 & .0967 & -.5944 & .0114 \\
\hline 13 & $1-3,125-131$ & .9559 & .8910 & .0764 & .3274 & .0433 & -.2171 & -.0100 \\
\hline 14 & $1-4,10-13$ & .9967 & .8835 & .0437 & .3835 & .1540 & -.2065 & .0196 \\
\hline 15 & $1-4,20-23$ & .9024 & .8592 & .0999 & .2102 & .1634 & -.2322 & .0098 \\
\hline 16 & $1-4,40-43$ & .9811 & .8964 & $.140 \mathrm{CO}$ & .3183 & .0630 & -.2288 & -.0176 \\
\hline 17 & $1-4,54-57$ & .9944 & .0704 & .0445 & .6762 & .1034 & -.2738 & .0023 \\
\hline 18 & $1-4,72-78$ & .9837 & .7535 & .0256 & .5022 & .0591 & -.3994 & .0075 \\
\hline 19 & $1-4,80-93$ & .9354 & .4829 & .0179 & .5297 & .1612 & -.6329 & .0282 \\
\hline 20 & $1-4,105-109$ & .9794 & .4812 & .0151 & .3131 & .0775 & -.2336 & .0062 \\
\hline 21 & $1-5,20-23$ & .9970 & .5900 & .0334 & .7749 & .1239 & -.1783 & .0135 \\
\hline 22 & $1-5,90-97$ & .9867 & .0071 & -.0121 & .9924 & .0389 & .0082 & -.0044 \\
\hline 23 & $1-5,144-147$ & .9954 & .8094 & .0302 & .4951 & .0812 & -.2960 & .0086 \\
\hline 24 & $2-1,3-6$ & .9933 & .9190 & .0610 & .2187 & .1288 & -.2834 & .0169 \\
\hline 25 & $2-1,10-16$ & .9838 & .8962 & .0359 & .3745 & .1012 & -.1092 & .0178 \\
\hline 26 & $2-1,20-23$ & .9909 & .7427 & $.037 c$ & .0313 & .0858 & -.1772 & .0041 \\
\hline 27 & $2-1,55-58$ & .9718 & .7549 & .0330 & .5824 & .0659 & -.2395 & .0039 \\
\hline 28 & $2-1,102-108$ & .9851 & .9652 & .0593 & .1369 & .0595 & .1665 & .0071 \\
\hline 29 & $2-1,135-138$ & .9775 & .8301 & .0277 & .3884 & .1942 & -.3130 & .0320 \\
\hline 30 & $2-2,10-13$ & .9687 & .9702 & .0302 & .1155 & .1130 & -.0047 & .0182 \\
\hline 31 & $2-2,20-23$ & .9653 & .9702 & .0451 & .0930 & .1071 & $-.02 \times 8$ & .0070 \\
\hline 32 & $2-2,56-63$ & .9653 & .0513 & .0291 & .3712 & .0906 & -.3037 & .0206 \\
\hline 33 & $2-2,95-98$ & .9708 & .9668 & .0463 & .1227 & .0455 & .1297 & .0019 \\
\hline 34 & $2-2,100-107$ & .9835 & .2015 & .0003 & .9684 & .0534 & -.0467 & -.0043 \\
\hline 35 & $2-2,130-13 ?$ & .9948 & .2745 & -.0020 & .9534 & .0544 & -.0863 & -.0005 \\
\hline 36 & $2-3,15-18$ & .9061 & .2670 & .0006 & .9584 & .0432 & -.0661 & -.0046 \\
\hline 37 & $2-3,58-65$ & .9703 & .9467 & .0405 & .1231 & .0712 & -.2282 & .0087 \\
\hline 38 & $2-3,93-96$ & .9355 & .9192 & .0217 & .1547 & .0322 & -.2548 & .0140 \\
\hline 39 & $2-3,107-113$ & .9585 & .9634 & .0609 & .0322 & .1348 & .0006 & .0247 \\
\hline 40 & $2-3,132-135$ & .9947 & .6757 & .0121 & .7122 & .0734 & -.1592 & .0080 \\
\hline 41 & $2-4,23-30$ & .9830 & .0773 & -.0097 & .9876 & .0371 & -.0090 & -.0048 \\
\hline 42 & $2-4,50-53$ & .9714 & .5626 & .0205 & .8072 & -.0257 & -.0417 & -.0270 \\
\hline 43 & $2-4,74-77$ & .9913 & .5190 & .0043 & .3446 & .0450 & -.0750 & -.0002 \\
\hline 44 & $2-4,120-177$ & .9880 & .0225 & -.0127 & .9929 & .0431 & -.0066 & -.0033 \\
\hline 45 & $2-5,10-13$ & .9879 & .7313 & .0196 & .6326 & .0719 & -.2174 & .0073 \\
\hline 46 & $2-5,20-23$ & .9946 & .7710 & .0238 & .6081 & .1133 & -.1286 & .0203 \\
\hline 47 & $2-5,50-57$ & .9020 & .0090 & -.0121 & .9398 & .0442 & -.0005 & -.0033 \\
\hline 48 & $2-5,90-83$ & .9859 & .9135 & .0319 & .2803 & .2391 & -.1131 & .0423 \\
\hline 49 & $2-6,20-23$ & .9926 & .8490 & .0228 & .4859 & .0606 & -.1776 & .0079 \\
\hline 50 & $2-6,64-71$ & .9947 & .5177 & -.0024 & .8509 & -.0061 & .0512 & -.0079 \\
\hline 51 & $2-6,103-110$ & .9897 & .9744 & .0302 & .1487 & .1120 & .0650 & .0225 \\
\hline 52 & $3-1,2-5$ & .9817 & .9639 & .0261 & .1252 & $.1 \mathrm{CJ} 1$ & -.1597 & .0270 \\
\hline 53 & $3-1,7-14$ & .9822 & .0534 & -.0113 & .9882 & .0516 & -.0016 & -.0011 \\
\hline 54 & $3-1,40-41$ & .9916 & .3031 & .0004 & .9392 & .0758 & -.1088 & .0031 \\
\hline 55 & $3-1,89-92$ & .9810 & .7046 & $.01 \cup 7$ & .6775 & . 0371 & -.1592 & -.0030 \\
\hline 56 & $3-2,40-41$ & .9938 & .9350 & .0175 & .3206 & .0410 & .1212 & .0083 \\
\hline 57 & $3-2,51-54$ & .9851 & .0160 & -.0126 & .9913 & .0433 & .0103 & -.0031 \\
\hline 58 & $3-2,89-96$ & .9844 & .7966 & .0053 & .5882 & .0437 & .0356 & .0126 \\
\hline 59 & $3-2,115-118$ & .9828 & .9581 & .0257 & .0904 & .2335 & .0051 & .0535 \\
\hline 60 & $3-2,138-139$ & .9763 & .9628 & .0214 & .0384 & .2103 & -.0273 & .0498 \\
\hline 61 & $3-2,147-150$ & .9305 & .9619 & .0850 & .3353 & .1695 & -.1316 & .0268 \\
\hline 62 & $3-3,29-32$ & .9665 & .8757 & .0357 & .0893 & .3786 & -.1992 & .0854 \\
\hline 63 & $3-3,41-48$ & .9724 & .9029 & .0320 & .3804 & .0975 & -.0409 & .0181 \\
\hline 64 & $3-3,70-71$ & .9017 & .6596 & $.04 y 8$ & .2711 & .3997 & -.4746 & .0753 \\
\hline 65 & $3-3,99-102$ & .9300 & .7730 & .0463 & .1440 & .5134 & -.1845 & .1093 \\
\hline 66 & $3-3,132-139$ & .9281 & .8475 & .0742 & .0921 & .4298 & -.0618 & .0849 \\
\hline 67 & $3-4,1-4$ & .9846 & .6563 & .1505 & .7271 & .0453 & -.0116 & -.0212 \\
\hline 68 & $3-4,47-48$ & .8857 & .5506 & .0348 & .2824 & .5528 & -.4276 & .1149 \\
\hline 69 & $3-4,64-67$ & .9941 & .4630 & .0114 & .3500 & .1681 & -.1678 & .0262 \\
\hline 70 & $3-4,105-110$ & .9828 & .6836 & .0363 & .6159 & .3296 & -.1501 & .0604 \\
\hline 71 & $3-5,16-19$ & .9965 & .3399 & .0078 & .9260 & .1349 & -.0709 & .0159 \\
\hline 72 & $3-5,47-48$ & .9609 & .6037 & .0206 & .0566 & .2243 & -.1918 & .0413 \\
\hline 73 & $3-5,00-03$ & .9786 & .9270 & .0353 & .0973 & .3126 & .0712 & .0682 \\
\hline 74 & $3-5,120-1 ? 7$ & .9836 & .0405 & -.0111 & .9900 & .0415 & .0059 & -.0041 \\
\hline 75 & $3-6,0-7$ & .9834 & .0693 & -.0095 & .9870 & .0660 & -.0035 & .0008 \\
\hline 76 & $3-6,40-49$ & .9827 & .5782 & .0443 & .7247 & .2471 & -.2421 & .0392 \\
\hline 77 & $3-6,80-84$ & .9930 & .8044 & .0329 & .5336 & .1884 & -.1530 & .0352 \\
\hline 78 & 3,66 & .9942 & .5183 & .0312 & .3443 & .1069 & -.0159 & .0066 \\
\hline
\end{tabular}


Estimated Superficial Temperatures of Winter $\left(T_{\mathrm{w}}\right)$ and Summer $\left(T_{\mathrm{s}}\right)$ of Hole 549A

APPENDIX C

Estimated Superficial Temperatures of Winter $\left(T_{\mathrm{w}}\right)$ and Summer $\left(T_{\mathrm{s}}\right)$ of Hole 548

(Cores 7-16)

\begin{tabular}{|c|c|c|c|}
\hline Sample & $\begin{array}{l}\text { Core-Section } \\
\text { (depth in } \mathrm{cm} \text { ) }\end{array}$ & $T_{\mathrm{w}}$ & $T_{\mathrm{S}}$ \\
\hline 1 & $7.1,76.79$ & $5 . t 9142$ & 10.38523 \\
\hline 2 & $7.2,24.25$ & $3.7 \div 310$ & c. 21204 \\
\hline 3 & 7.3 .104 .111 & $5.914<2$ & 11. $t 0215$ \\
\hline 4 & $7.4,40.43$ & 5.82644 & 11.20102 \\
\hline 5 & 7.4 .103 .117 & 7. $c \bar{c} \in 78$ & 12.86654 \\
\hline t & $7.5 .19 .2 \bar{c}$ & $t .8 \geq 6 ? 8$ & 12.15941 \\
\hline$\therefore$ & 7.5 .111 .116 & $1 C .4 C 5 E 1$ & 16.80219 \\
\hline 8 & 7.5 .140 .141 & 5.78023 & 11.40644 \\
\hline 9 & $7 \cdot t \cdot 11 c \cdot 117$ & $9.16=55$ & $14.8 \geq 1 \in 6$ \\
\hline 10 & 7.7 .10 .13 & 7.65740 & 13.10918 \\
\hline 11 & $8.1, t 5.7 t$ & 7. $527 \overline{11}$ & 12.69859 \\
\hline 12 & $\varepsilon .1,1 z z .13 c$ & 9.72078 & 15.91424 \\
\hline 13 & 8.2 .40 .47 & 9.25829 & 14.35469 \\
\hline 14 & $8 . \bar{c}, 9[.91$ & 8.59829 & 14.71252 \\
\hline 15 & $8, i, 14 C, 167$ & $\varepsilon .26912$ & 13.43070 \\
\hline 16 & $\varepsilon . ?, t 5.7 \mathrm{C}$ & $1 C . ; c 8 \leq 2$ & $16.841 \mathrm{C6}$ \\
\hline 16 & .27 & 12.24509 & 19.40244 \\
\hline 18 & 8.4 .90 .91 & $8.489 C 1$ & 13.95873 \\
\hline 19 & $8.5,00.27$ & 9.75616 & 16.45871 \\
\hline 20 & 8.5 .69 .75 & 12.19739 & 20.19257 \\
\hline 21 & \&. : & 5.53822 & 10.40253 \\
\hline 22 & $8 . t .69 .70$ & $8 . t 3552$ & 15.48079 \\
\hline 23 & e.t.124.125 & 6.92314 & 11.82453 \\
\hline 24 & 1.20 .22 & 8. $¿ 86 \overline{c 1}$ & 14.41473 \\
\hline 25 & $9=1,10 \mathrm{C}=107$ & 11.56480 & 19.09677 \\
\hline 26 & $9.2,20.23$ & 13. $a C \in C 7$ & 21.32697 \\
\hline 27 & 9.2 .43 .48 & 13.15438 & 21.27541 \\
\hline 28 & 9.3, & 13. & 21. \\
\hline 29 & $9.3,20.23$ & 12.53332 & 20.79773 \\
\hline & $4,43.44$ & $4.8 \leq 4 c 2$ & $10.600 ? 6$ \\
\hline & & & 13.58 \\
\hline 32 & $10.5,43.44$ & & 11.93618 \\
\hline 33 & $10.5,120.122$ & 5.364 & 10.64141 \\
\hline & 11. & 11.11 & $18.2 \mathrm{C}$ \\
\hline 35 & 11.1 & 10.2 & \\
\hline $3 t$ & $11.2,=0 . \leq 3$ & 11.12970 & 18.87744 \\
\hline 37 & $11.2,140.147$ & 11. 26942 & 19.3829 .5 \\
\hline & & 5.27 & 16.13654 \\
\hline 39 & $11.3,139.146$ & 1C. C 2721 & 17.34154 \\
\hline 40 & $11.4,140.147$ & $\varepsilon .3 \varepsilon<45$ & $14 . t \in 243$ \\
\hline 41 & & & \\
\hline 42 & $11.5,50.53$ & 12.77940 & 21.57589 \\
\hline 43 & $11.5,52.57$ & 11.91446 & 18.03188 \\
\hline 34 & & & \\
\hline 45 & 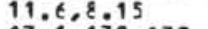 & 12. & 40 \\
\hline $4 t$ & $1 \geq 7$ & 13. & $21.213 \in 5$ \\
\hline 47 & 13 & 12.4 & 20.26924 \\
\hline 48 & & & \\
\hline 39 & .29 & c. 123 & 17.92125 \\
\hline 50 & 13. & t. $z t \epsilon$ & 13.03616 \\
\hline & & & \\
\hline 52 & & & \\
\hline 53 & $13.6,27.36$ & $1 . C \geq 1 z 1$ & 7.CहO 24 \\
\hline & & & \\
\hline & & $1 \mathrm{C}$. & \\
\hline $5 t$ & $15 \cdot 1,1$ & $16 . \leq \leq \varepsilon \leq 5$ & $17.629 \mathrm{c} 1$ \\
\hline 57 & 15.2 & & \\
\hline & & & \\
\hline 59 & & 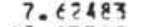 & 13.55 \\
\hline 60 & 15 & 12. & 35 \\
\hline & & & \\
\hline & & 12 & \\
\hline$t^{3}$ & & 9.4 & 16. \\
\hline 64 & it & 8.8 & 1 \\
\hline & & & \\
\hline 66 & & $7.827<3$ & 13.3 \\
\hline 67 & $14 \mathrm{C} .141$ & 7.13766 & 13.03857 \\
\hline
\end{tabular}
(Cores 1-3)

\begin{tabular}{|c|c|c|c|}
\hline Sample & $\begin{array}{l}\text { Core-Section } \\
\text { (depth in } \mathrm{cm} \text { ) }\end{array}$ & $T_{\mathrm{W}}$ & $T_{\mathrm{S}}$ \\
\hline 1 & $1-1,1-4$ & 10.49293 & 16.93039 \\
\hline 2 & $1-1,8-11$ & 9.66333 & 15.65680 \\
\hline 3 & $1-1,39-42$ & 6.43232 & 10.34090 \\
\hline 4 & $1-1,42-45$ & 1.47280 & 7.22030 \\
\hline 5 & $1-1,137-140$ & 3.01429 & 8.23057 \\
\hline 6 & $1-2,2-5$ & 3.55543 & 9.78546 \\
\hline 7 & $1-2,5-8$ & $2.89 \geq 84$ & 9.10310 \\
\hline 8 & $1-2,70-77$ & -.14377 & 5.77677 \\
\hline 9 & $1-2,130-137$ & 3.32341 & 8.74736 \\
\hline 10 & $1-3,10-13$ & 6.62611 & 11.30172 \\
\hline 11 & $1-3,20-23$ & 7.86067 & 12.34632 \\
\hline 12 & $1-3,70-77$ & 5.04178 & 11.0 \\
\hline 13 & $1-3,125-131$ & 8.94804 & 13.95925 \\
\hline 14 & $1-4,10-13$ & 9.17361 & 14.24243 \\
\hline 15 & $1-4,20-23$ & 9.86594 & 15.42049 \\
\hline 16 & $1-4,40-43$ & 10.92645 & 16.04713 \\
\hline 17 & $1-4,54-57$ & 9.71626 & 13.21944 \\
\hline 18 & $1-4,72-78$ & $0.7 \mathrm{C3CJ}$ & 11.39479 \\
\hline 19 & $1-4,88-93$ & 4.30582 & 10.78611 \\
\hline 20 & $1-4,105-109$ & 7.63444 & 12.22343 \\
\hline 21 & $1-5,20-23$ & 9.16980 & 13.75793 \\
\hline 22 & $1-5,90-97$ & .06705 & 5.85265 \\
\hline 23 & $1-5,144-147$ & 7.85087 & 12.35516 \\
\hline 24 & $2-1,3-6$ & 9.09218 & 14.57053 \\
\hline 25 & $2-1,1 i-16$ & 8.31856 & 14.10440 \\
\hline 26 & $2-1,20-23$ & 9.41603 & 13.57013 \\
\hline 27 & $2-1,55-58$ & 8.32694 & 12.92261 \\
\hline 28 & $2-1,1 \mathrm{~J} 2-1 \mathrm{~J} 8$ & 9.54050 & 16.95036 \\
\hline 29 & $2-1,135-138$ & 7.37357 & 12.78659 \\
\hline 30 & $2-2,10-13$ & 9.50096 & 16.26984 \\
\hline 31 & $2-2,20-23$ & 9.77334 & 16.20111 \\
\hline 32 & $2-2,56-63$ & 7.23362 & 12.81685 \\
\hline 33 & $2-2,95-95$ & 9.07492 & 16.12820 \\
\hline 34 & $2-2,100-107$ & 4.55593 & 9.20292 \\
\hline 35 & $2-2,130-1 \geq 3$ & 5.39832 & 10.22379 \\
\hline 36 & $2-3,15-18$ & 5.65379 & 10.24031 \\
\hline 37 & $2-3,58-65$ & 8.83380 & 14.32741 \\
\hline 38 & $2-3,93-96$ & 7.62442 & 13.25382 \\
\hline 39 & $2-3,107-113$ & 10.97058 & 18.04550 \\
\hline 40 & $2-3,132-135$ & 8. 20077 & 12.62355 \\
\hline 41 & $2-4,23-30$ & 1.58940 & 7.09726 \\
\hline 42 & $2-4,50-53$ & 9.74671 & 12.14389 \\
\hline 43 & $2-4,74-77$ & 7.79979 & 12.02354 \\
\hline 44 & $2-4,120-177$ & .37858 & 6.25343 \\
\hline 45 & $2-5,10-13$ & 8.17858 & 12.58294 \\
\hline 40 & $2-5,20-23$ & 8.43251 & 13.82546 \\
\hline 47 & $2-5,50-57$ & .09756 & 5.89636 \\
\hline 48 & $2-5,80-83$ & 9.43759 & 15.53769 \\
\hline 49 & $2-6,23-23$ & 7.94250 & 12.97385 \\
\hline 50 & $2-6,64-71$ & 6.88890 & 11.64618 \\
\hline 51 & $2-6,103-110$ & 9.40552 & 10.60504 \\
\hline 52 & $3-1,2-5$ & 9.14360 & 15.45679 \\
\hline 53 & $3-1,7-16$ & .72737 & 6.48319 \\
\hline 54 & $3-1,40-41$ & 5.83544 & 10.40769 \\
\hline 55 & $3-1,89-92$ & 7.79130 & 12.54321 \\
\hline 56 & $3-2,4[-41$ & 7.62858 & 14.54194 \\
\hline 57 & $3-2,51-54$ & .04773 & 5.85493 \\
\hline 58 & $3-2,89-96$ & 7.24803 & 13.42494 \\
\hline 59 & $3-2,115-110$ & 10.90964 & 18.47916 \\
\hline $6 i$ & $3-2,138-1 \geq 9$ & 11.31303 & 18.64226 \\
\hline 61 & $3-2,147-150$ & 11.93290 & 18.30708 \\
\hline 62 & $3-3,29-32$ & 11.64555 & 18.99684 \\
\hline 63 & $3-3,41-40$ & 0.39251 & 14.74516 \\
\hline 64 & $3-3,70-71$ & 7.26002 & 14.63716 \\
\hline 65 & $3-3,99-102$ & $10.8 C 454$ & 18.67522 \\
\hline 60 & $3-3,132-139$ & 12.45177 & 20.20093 \\
\hline 67 & $3-4,1-4$ & 15.02878 & 21.62447 \\
\hline 68 & $3-4,47-40$ & 6.52559 & 14.68621 \\
\hline 69 & $3-4,64-67$ & 6.84652 & 11.76618 \\
\hline 70 & $3-4,105-110$ & 8. 01063 & 13.32349 \\
\hline 71 & $3-5,10-19$ & 5.85319 & 10.70071 \\
\hline 72 & $3-5,47-48$ & 7.37600 & 12.62607 \\
\hline 73 & $3-5,3 J-33$ & 11.44243 & 19.47319 \\
\hline 74 & $3-5,120-127$ & .70023 & 6.29236 \\
\hline 75 & $3-0,0-7$ & 1. 09088 & 6.59861 \\
\hline 76 & $3-6,46-49$ & 8.25917 & 13.42511 \\
\hline 77 & $3-6,33-54$ & 8.65704 & 14.24666 \\
\hline 78 & $3, \mathrm{cc}$ & 9.07235 & 13.44048 \\
\hline
\end{tabular}



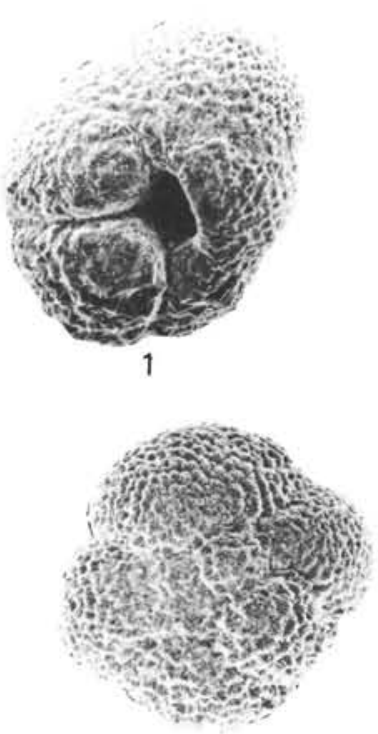

5
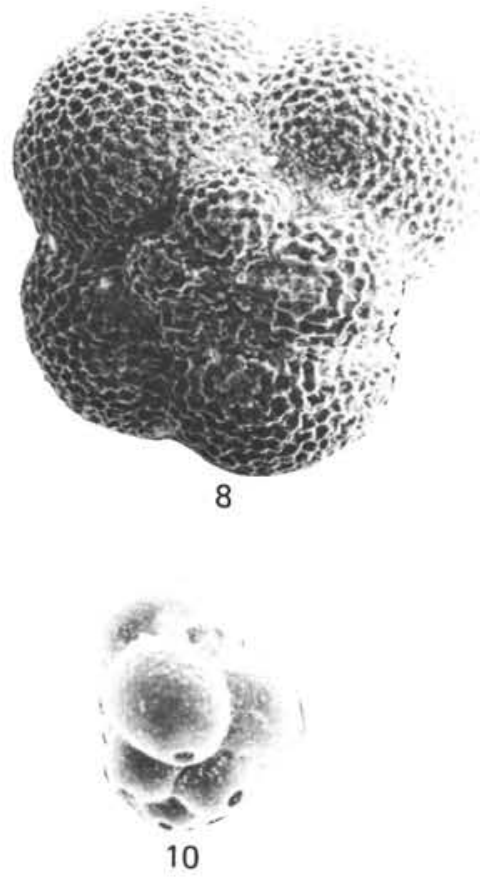
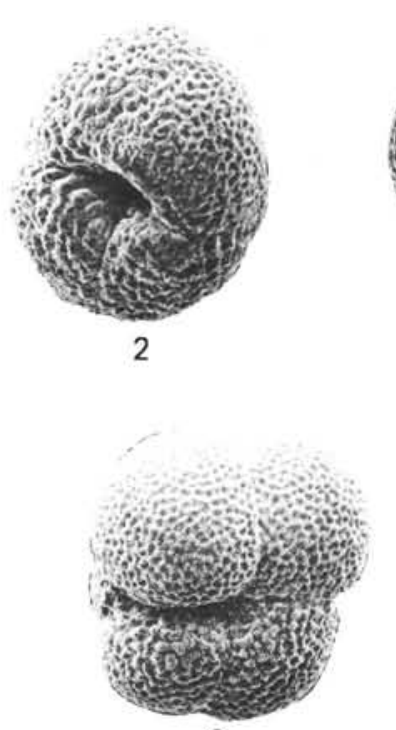

6
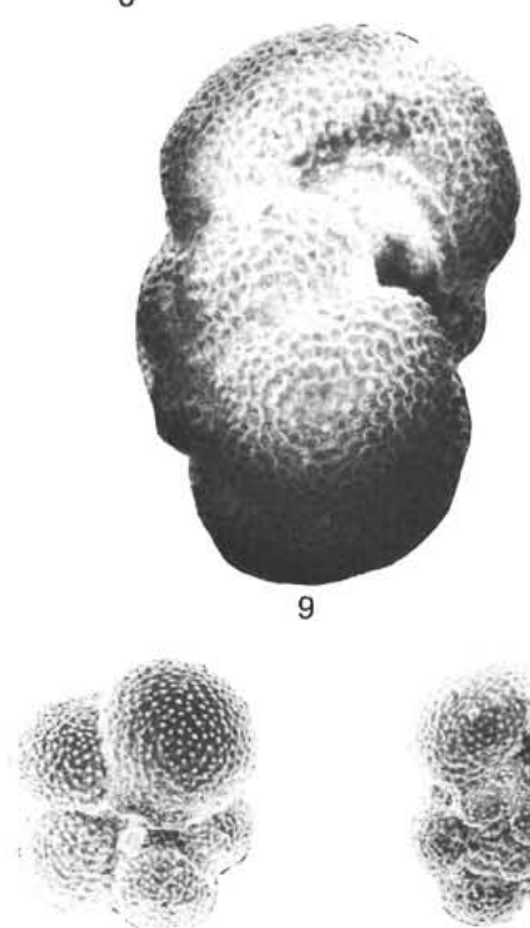

11
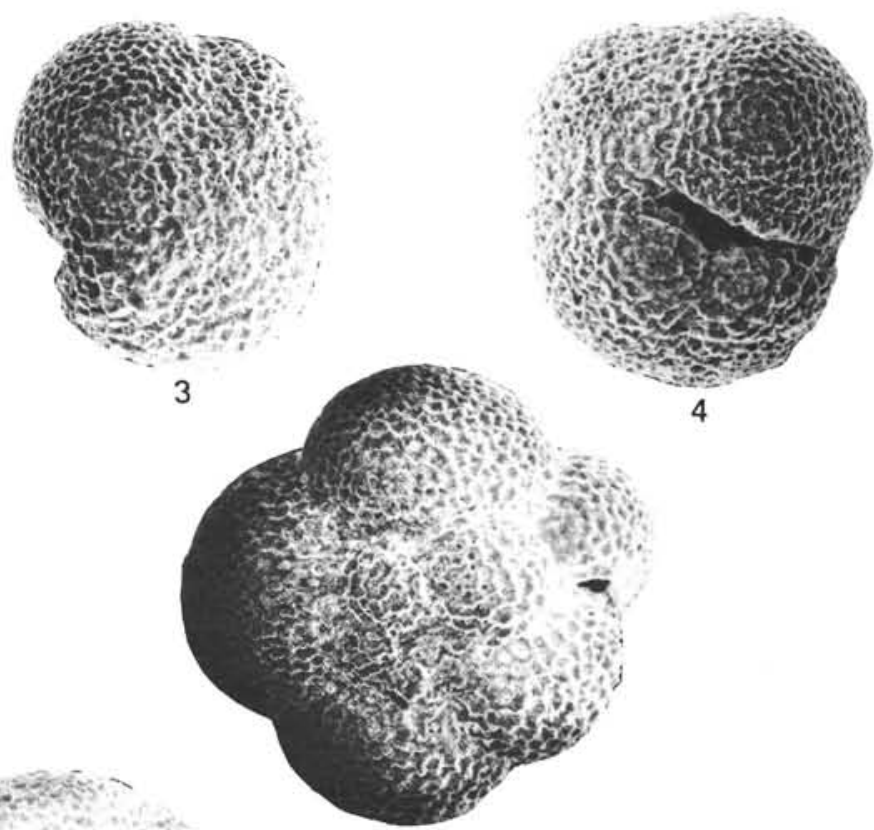

7

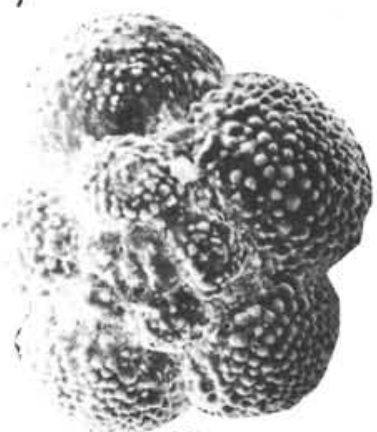

13

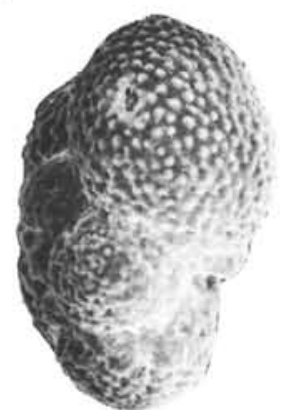

14

Plate 1. 1-4. Globigerina pachyderma (Ehrenberg), left-coiling. $(1,2,4)$ Sample 549A-1-2 $(70-72 \mathrm{~cm}), \times 120$, umbilical view. (3) Sample 549A-1-2 $(70-72 \mathrm{~cm}), \times 120$, spiral view. 5-9. Globigerina pachyderma (Ehrenberg), right-coiling. (5) Sample 549A-1-1 $(39-42 \mathrm{~cm}), \times 120$, spiral view. (6) Sample 549A-1-1 $(39-42 \mathrm{~cm}), \times 120$, umbilical view. $(7,8)$ Sample 549A-1-1 $(1-4 \mathrm{~cm}), \times 140$, spiral view. (9) Sample 549A-1-1 (1-4 cm), $\times 140$, side view. 10. Globigerinita uvula (Ehrenberg), Sample $549 \mathrm{~A}-1-1(39-42 \mathrm{~cm}), \times 160$, umbilical view. 11-14. Globigerina quinqueloba Natland. (11) Sample 549A-1-1 (39-42 cm), $\times 120$, umbilical view. (12) Sample 549A-1-1 (39-42 cm), $\times 120$, spiral view. (13) Sample 549A-1-1 $(1-4 \mathrm{~cm}), \times 200$, spiral view. (14) Sample 549A-1-1 $(1-4 \mathrm{~cm}), \times 200$, side view. 

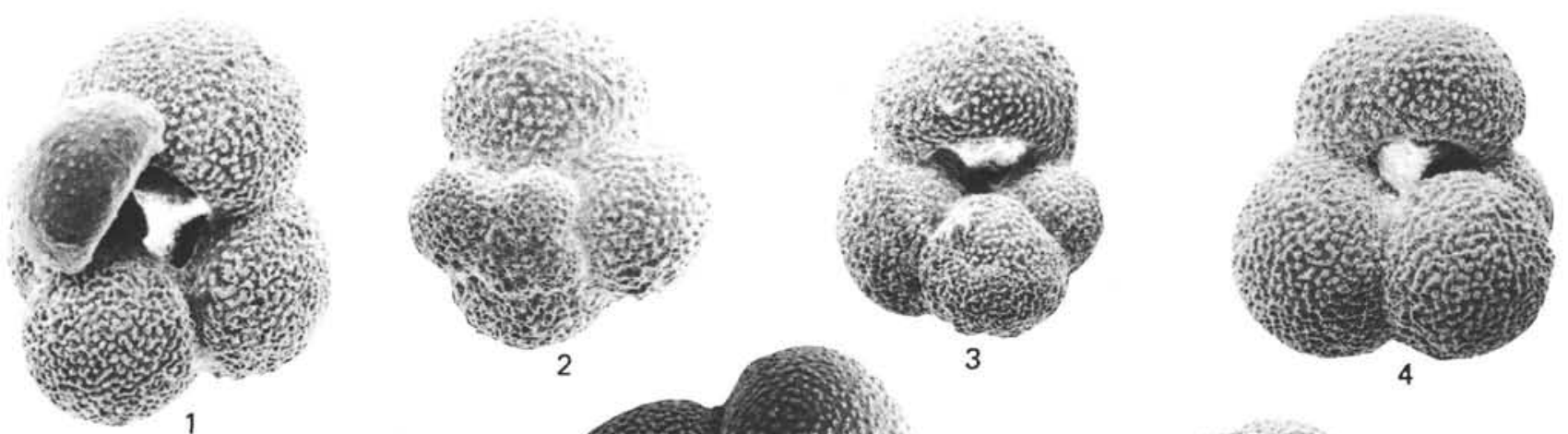

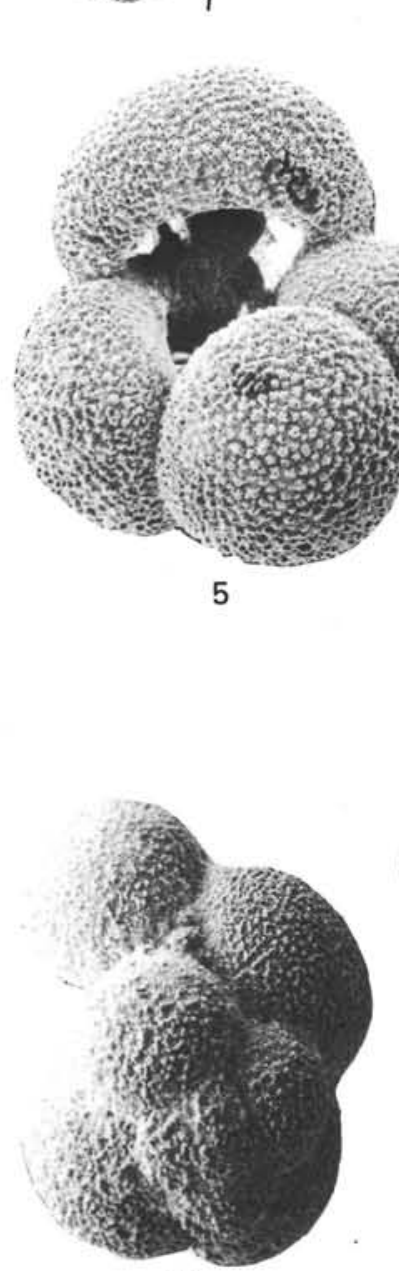

10

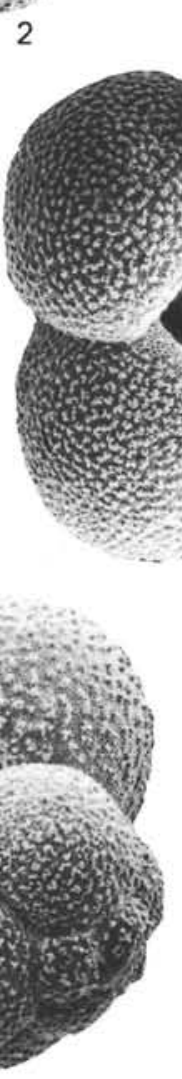

8
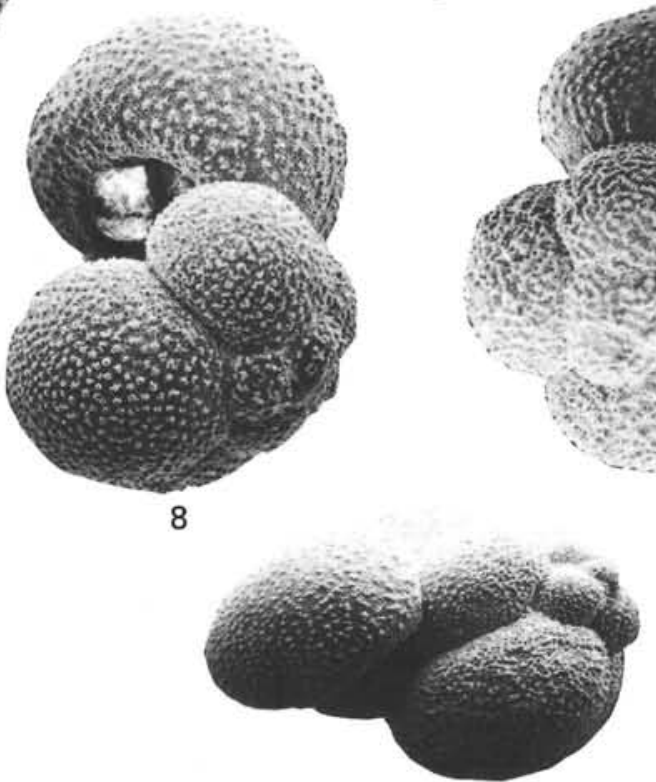

12

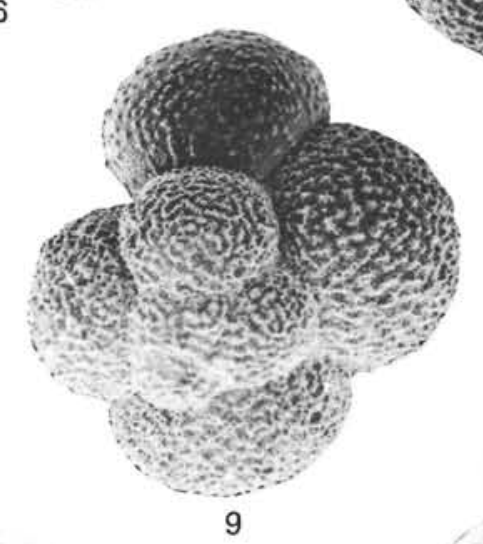

9

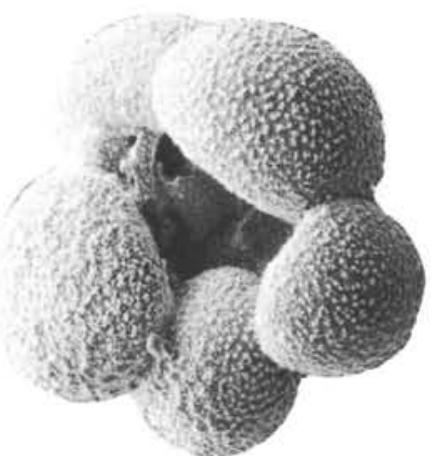

11

Plate 2. 1-4. Globigerina bulloides d'Orbigny. (1) Sample 549A-1-1 (39-42 cm), $\times 120$, umbilical view. (2) Sample 549A-1-1 (39-42 cm), $\times 120$, spiral view. (3) Sample 549A-1-1 (39-42 cm), × 100, umbilical view. (4) Sample 549A-3-3 $(132-134 \mathrm{~cm}), \times 100$, umbilical view. 5-12. Globigerina cariacoensis Rögl and Bolli. (5-7) Sample 549A-3-3 (132-134 cm), ×120, umbilical view. (8) Sample 549A-3-3 (132-134 cm), $\times 100$, side view. (9) Sample 549A-3-3 (132-134 cm), $\times 100$, spiral view. (10) Sample 548-8-1 $(120-122 \mathrm{~cm}), \times 80$, spiral view. (11) Sample 548-8-1 (120-122 cm), $\times 80$, umbilical view. (12) Sample 548-8-5 (20-22 cm), side view. 

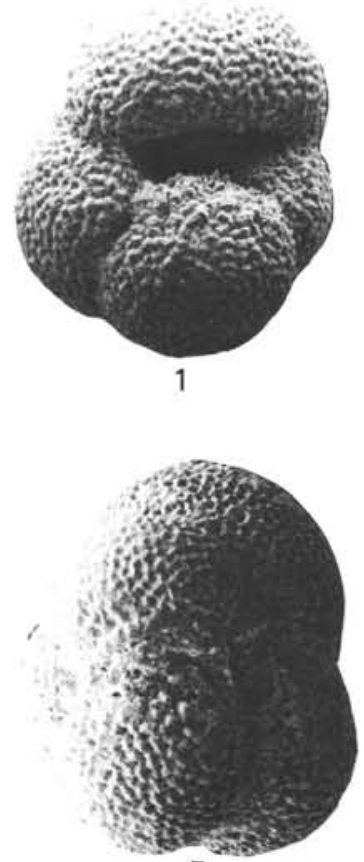

5
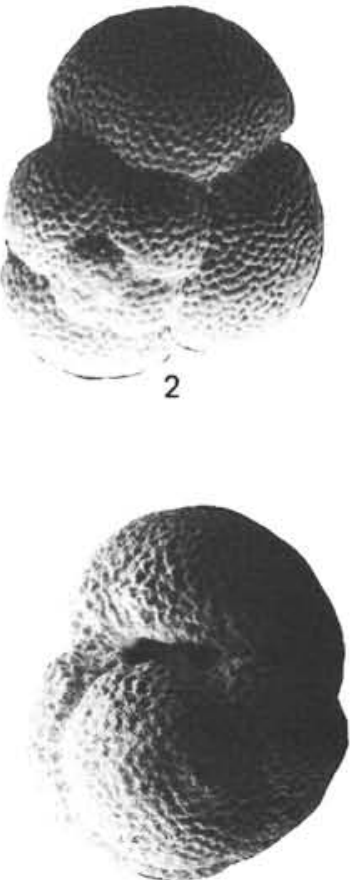

6

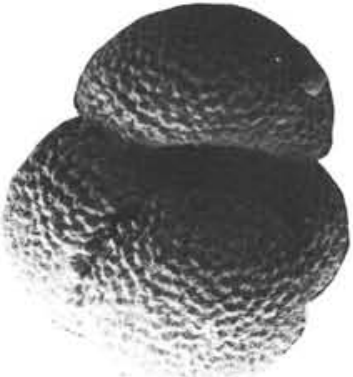

3

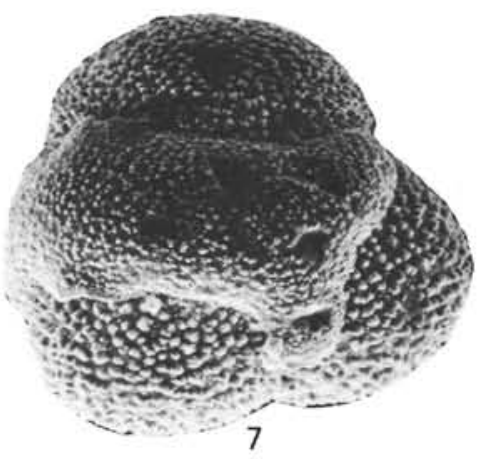

7

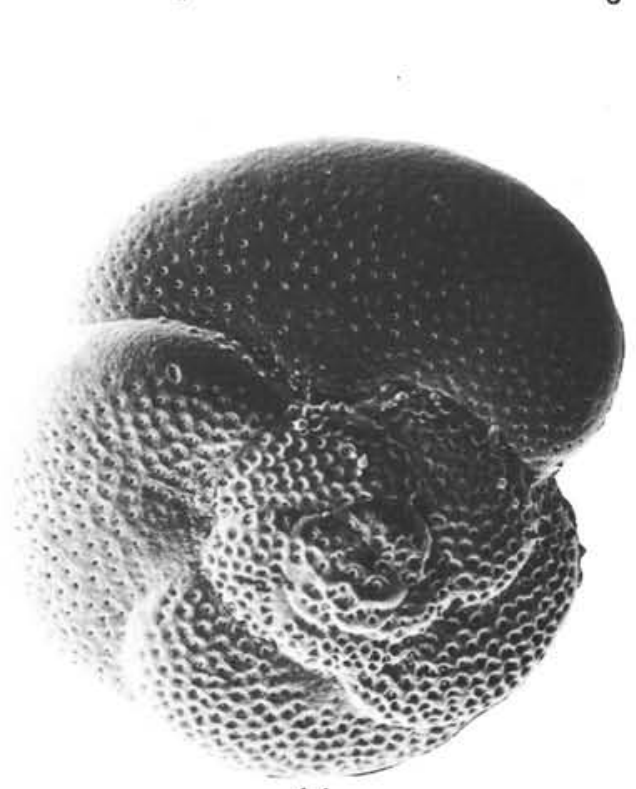

14

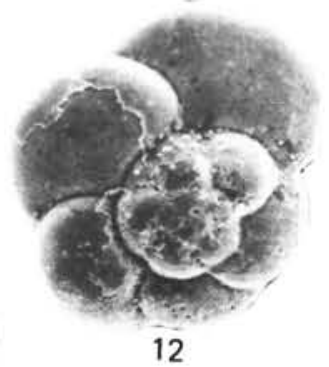

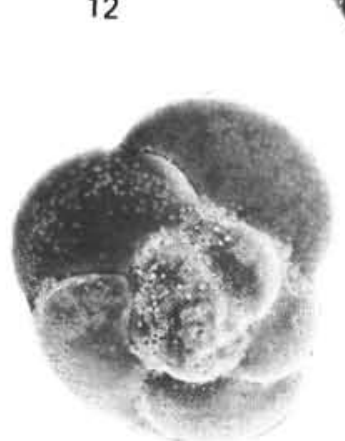

11

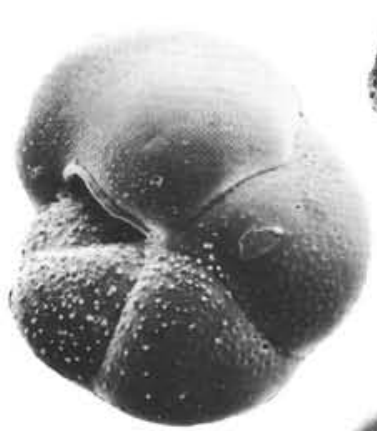

10

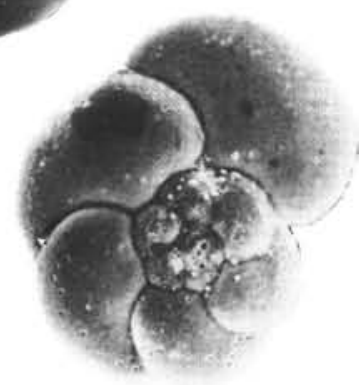

13

Plate 3. 1-6. Globigerina bulloides d'Orbigny var. (1) Sample 548-18-4 (25-27 cm), $\times 100$, umbilical view. (2) Sample 548-18-4 (25-27 cm), $\times 100$, spiral view. (3) Sample 548-19-3 (53-56 cm), $\times 100$, umbilical view. (4) Sample 548-29-3 (53-56 cm), $\times 100$, spiral view. (5) Sample 548-18-4 (25$27 \mathrm{~cm}), \times 100$, umbilical view. (6) Sample 548-19-3 (53-56 cm), $\times 100$, umbilical view. 7-9. Globigerinita glutinata (Egger). (7) Sample 548-21-3 $(37-39 \mathrm{~cm}), \times 200$, umbilical view. (8) Sample 549A-1-1 $(39-42 \mathrm{~cm}), \times 120$, umbilical view. (9) Sample 549A-1-1 $(39-42 \mathrm{~cm}), \times 140$, spiral view. 10, 13. Globorotalia scitula (Brady) form 1. Sample 549A-1-1 $(39-42 \mathrm{~cm}), \times 100$, umbilical view. (13) Sample $549 \mathrm{~A}-1-1(1-4 \mathrm{~cm}), \times 150$, spiral view. 11-12. Globorotalia bermudezi Rögl and Bolli. (11) Sample 549A-1-1 (39-42 cm), $\times 100$, spiral view. (12) Sample 549A-1-1 (1-4 cm), $\times 150$, spiral view. 14. Globorotalia scitula (Brady) form 2, Sample 549A-3-1 $(7-14 \mathrm{~cm}), \times 100$, spiral view. 


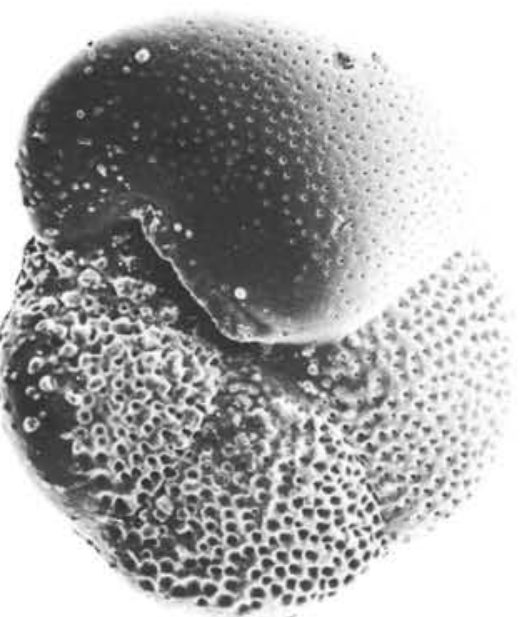

1

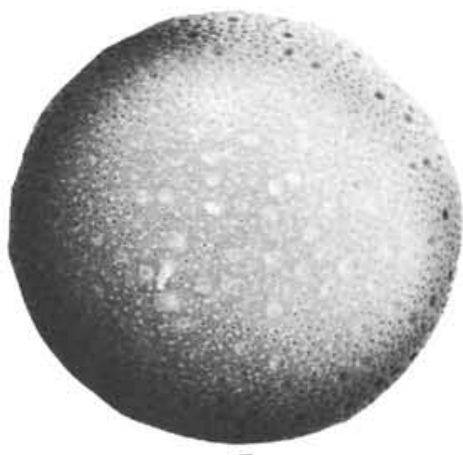

7
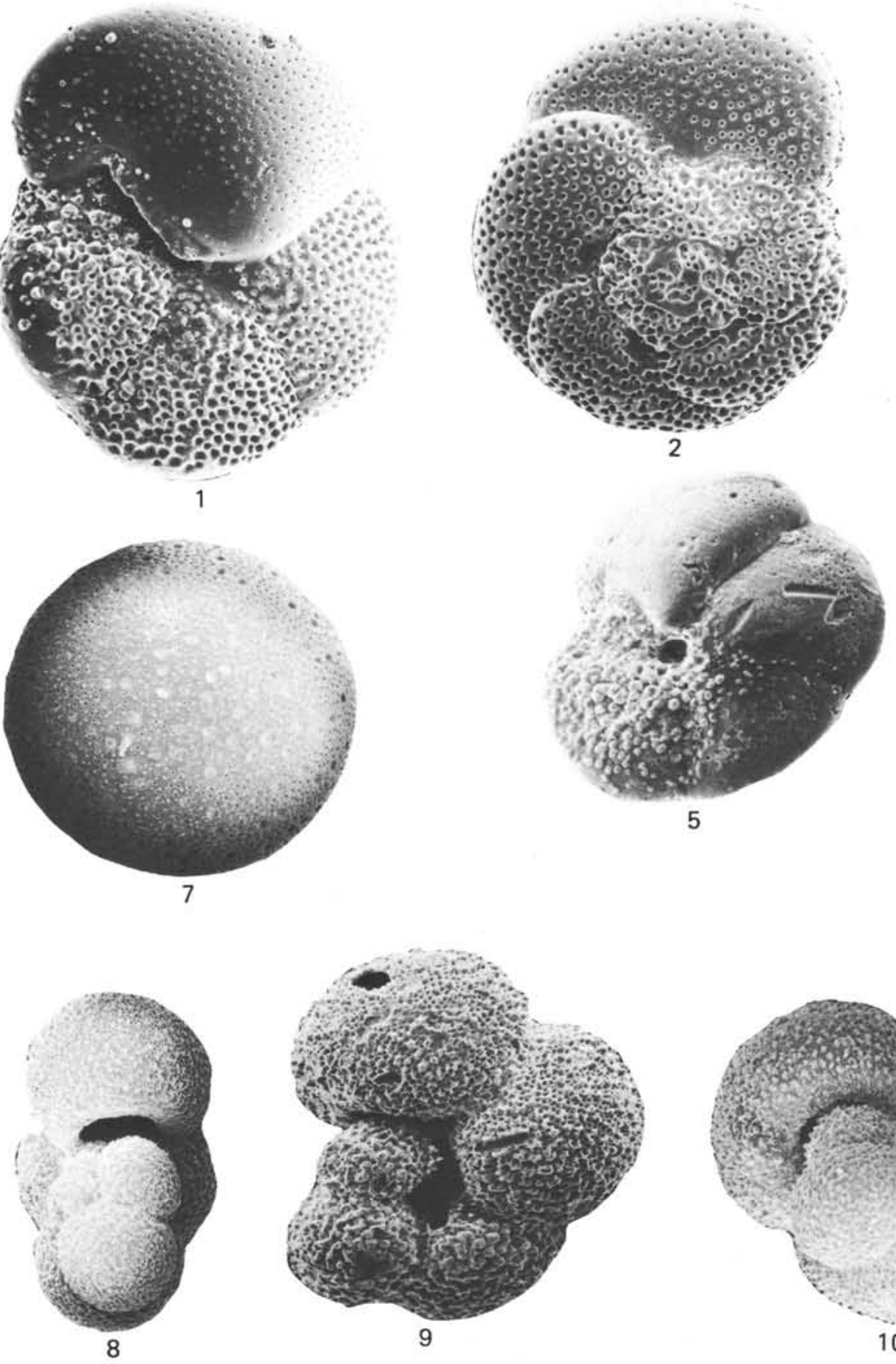

5
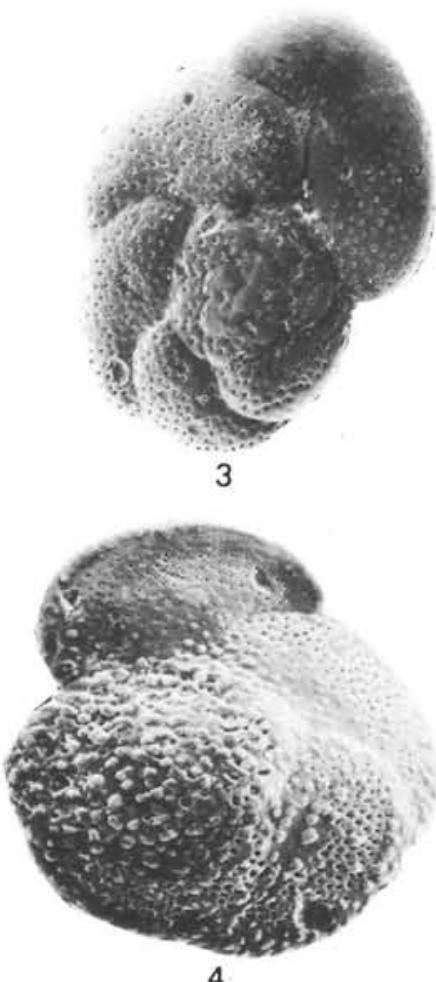

4

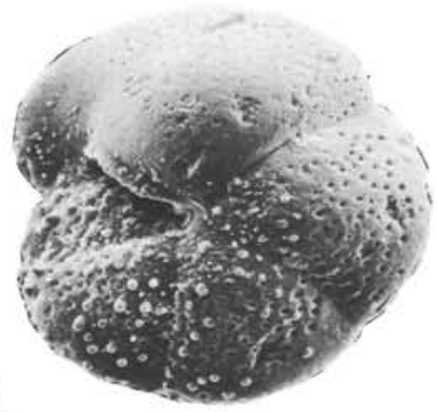

6

Plate 4. 1-2. Globorotalia scitula (Brady) form 2. (1) Sample 549A-3-3 (132-134 cm), $\times 100$, umbilical view. (2) Sample 549A-3-3 (132-134 cm), $\times 100$, spiral view. 3-6. Globorotalia scitula (Brady) form 1. (3) Sample 548-27,CC, $\times 100$, spiral view. (4) Sample 548-35,CC, $\times 140$, spiral view. (5) Sample 548-35,CC, $\times 140$, umbilical view. (6) Sample 548-35,CC, $\times 140$, umbilical view. 7. Orbulina universa d'Orbigny, Sample 549A-1-1 (39-42 cm), $\times 80$, 8-10. Hastigerina siphonifera (d'Orbigny). (8) Sample 549A-2-1 (3-6 cm), $\times 80$, peripheral view. (9) Sample 548-21$3(37-39 \mathrm{~cm}), \times 100$, side view. (10) Sample 549A-1-4 $(40-43 \mathrm{~cm}), \times 80$, peripheral view. 


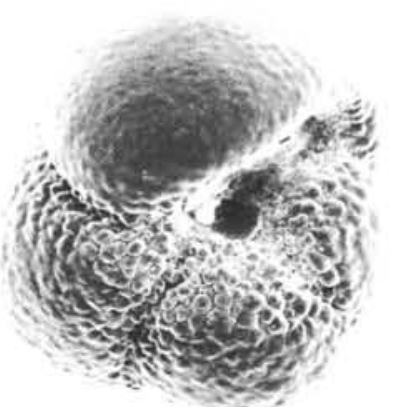

1

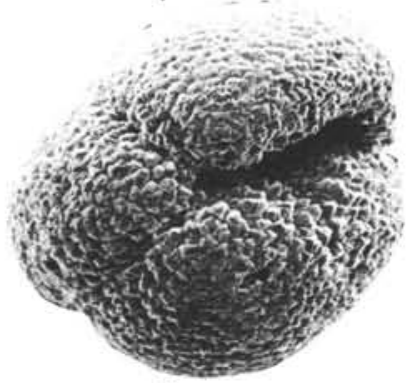

7

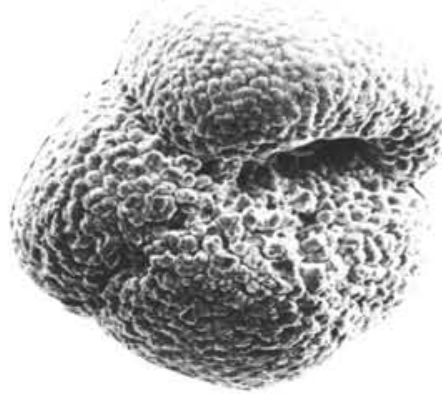

8

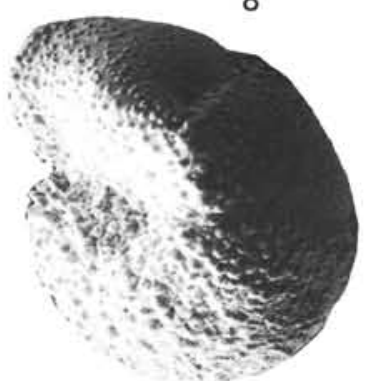

11
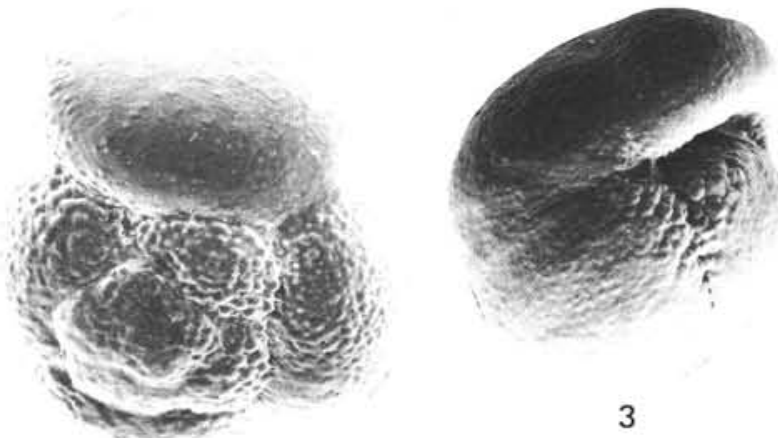

3
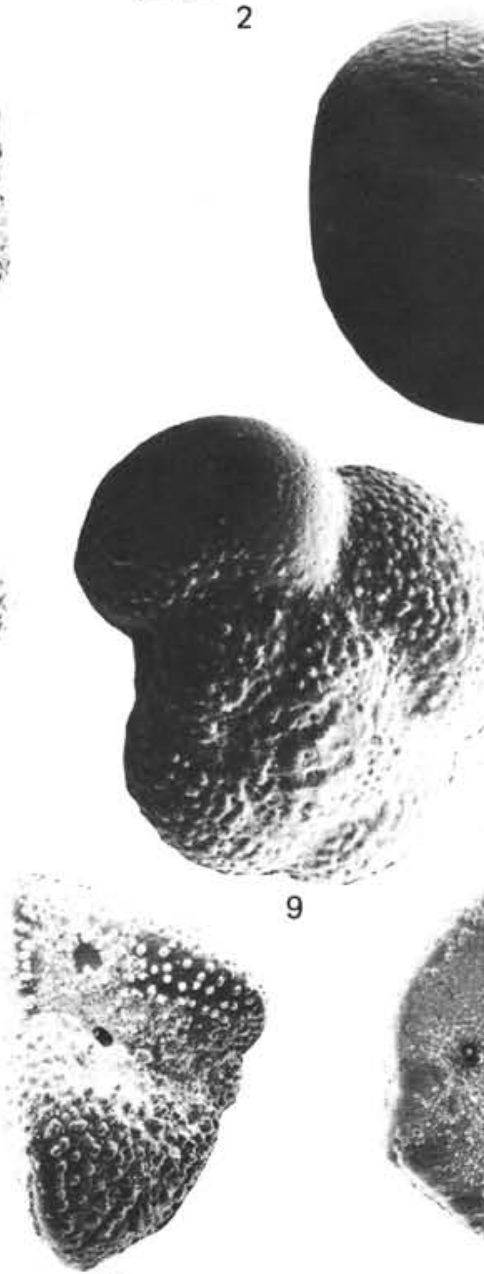

12

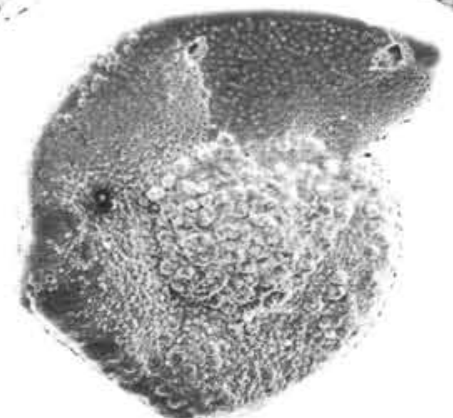

13

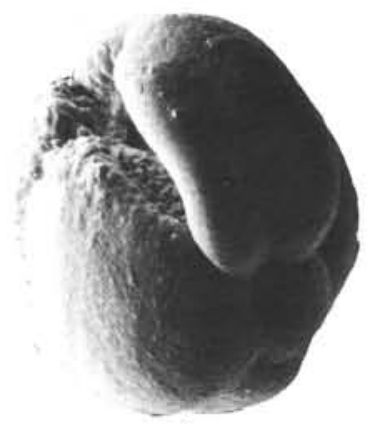

4

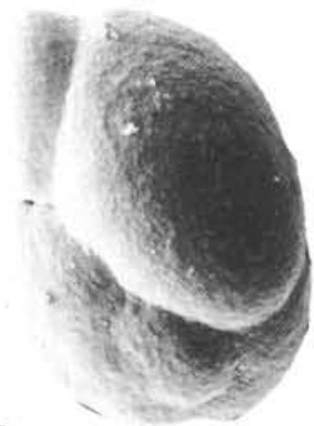

6

Plate 5. 1-2. Globorotalia inflata (d'Orbigny). (1) Sample 549A-1-1 $(39-42 \mathrm{~cm}), \times 100$, umbilical view. (2) Sample 549A-1-1 (39-42 cm), $\times 100$, spiral view. 3-6. Globorotalia inflata (d'Orbigny) var. (3) Sample 548-7-4 (103-110 cm), $\times 80$, umbilical view. (4) Sample 548-7-4 (103-110 cm), $\times 80$, side view. (5) Sample 548-7-4 (103-110 cm), $\times 80$, umbilical view. (6) Sample 548-7-4 $(103-110 \mathrm{~cm}), \times 80$, side view. 7-8. Globorotalia inflata ex. interc. G. puncticulata, Sample 548-21-3 $(37-39 \mathrm{~cm}), \times 100$, umbilical views. 9-10. Globorotalia aff. inflata (d'Orbigny), Sample 54818-3 (42-45 cm), $\times 160$, spiral views. 11-13. Globorotalia truncatulinoides (d'Orbigny). (11) Sample 548-12-1 $(129-130 \mathrm{~cm}), \times 100$, umbilical view. (12) Sample 549A-1-4 (40-43 cm), $\times 80$, side view. (13) Sample 549A-1-1 (39-42 cm), $\times 80$, spiral view. 14. Globorotalia aff. truncatulinoides, Sample 548-12-1 (123-130 cm), spiral view. 

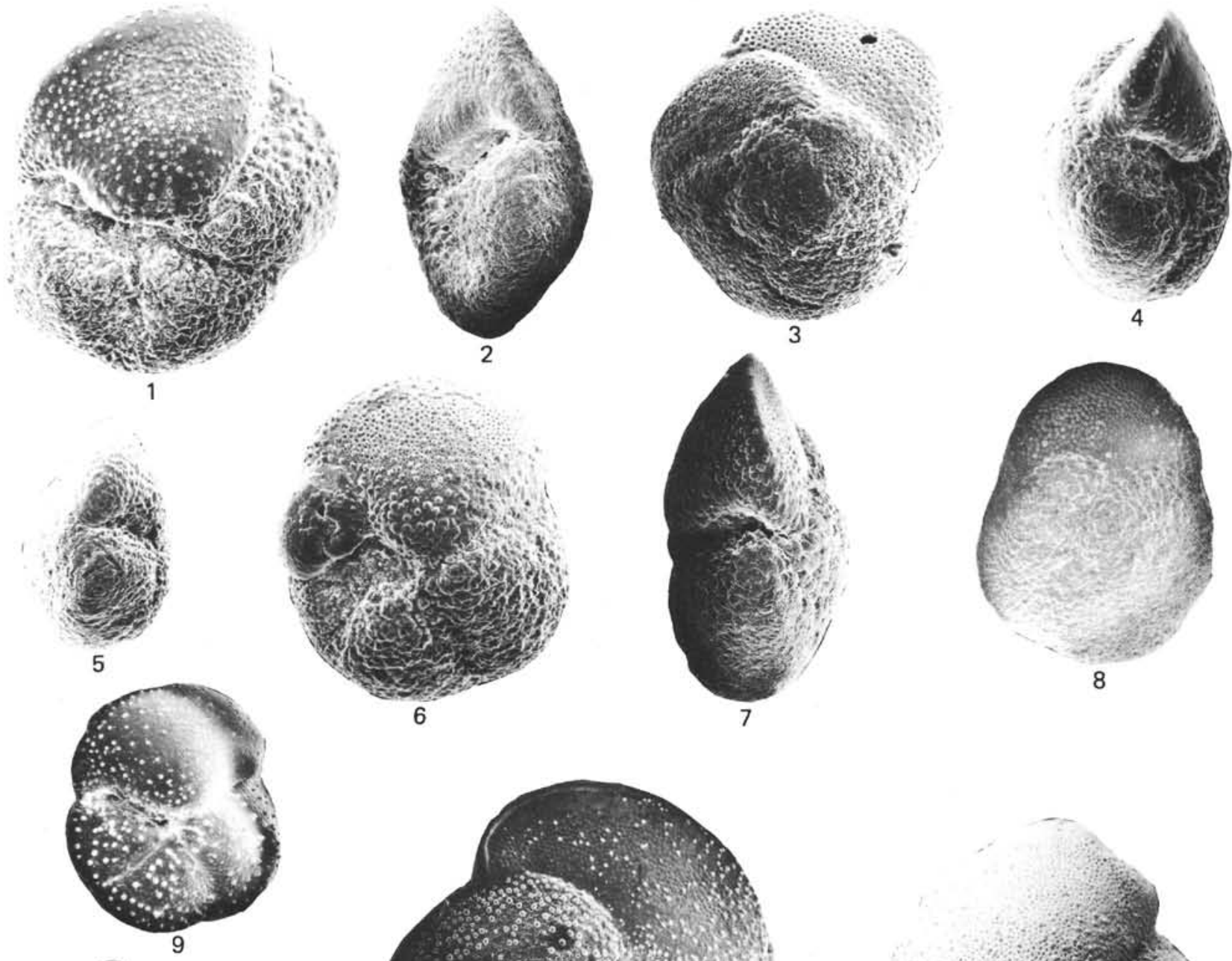

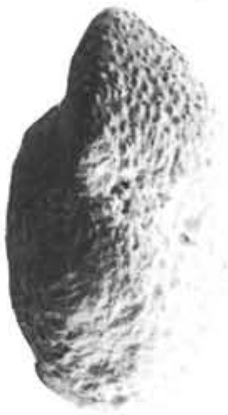

10

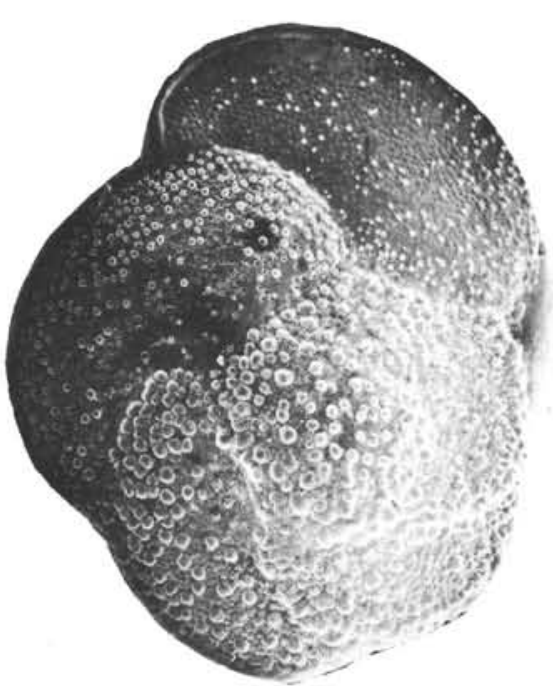

11

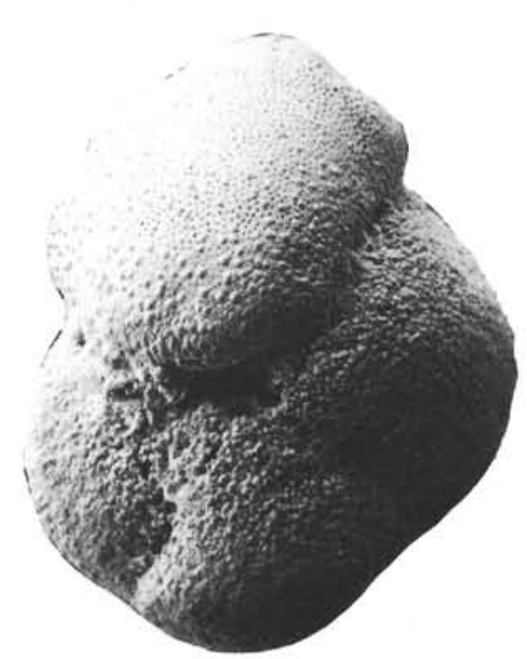

12

Plate 6. 1-12. Globorotalia hirsuta (d'Orbigny). (1) Sample 549A-1-1 $(39-42 \mathrm{~cm}), \times 80$, umbilical view. (2) Sample 549A-1-1 (39-42 cm), $\times 80$, side view. (3) Sample 549A-1-4 (40-43 cm), $\times 80$, spiral view. (4) Sample 549A-1-4 (40-43 cm), $\times 80$, side view. (5) Sample 550-1-3 (130-137 cm), $\times 80$, side view. (6) Sample 549A-1-4 (40-43 cm), $\times 80$, umbilical view. (7) Sample 549A-1-1 $(39-42 \mathrm{~cm}), \times 80$, side view. (8) Sample 550-1-3 (130-137 $\mathrm{cm}), \times 80$, spiral view. (9) Sample 549A-2-1 $(3-6 \mathrm{~cm}), \times 80$, umbilical view. (10) Sample 548-7-7 $(10-13 \mathrm{~cm}), \times 80$, side view. (11) Sample 549A-1-4 $(40-43 \mathrm{~cm}), \times 80$, spiral view. (12) Sample 548-7-7 $(10-13 \mathrm{~cm}), \times 80$, umbilical view. 


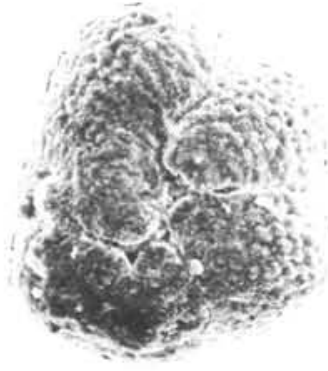

1

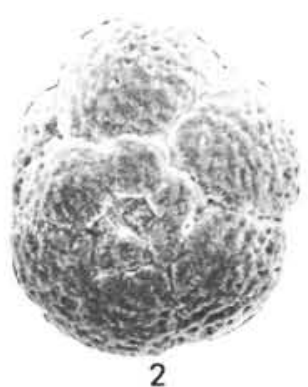

2
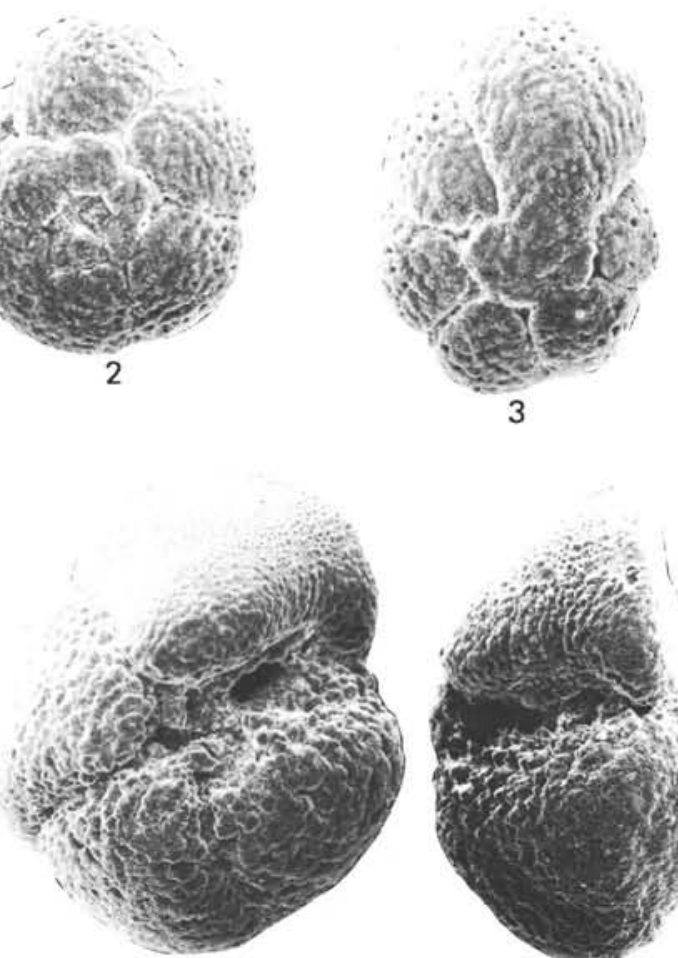

6

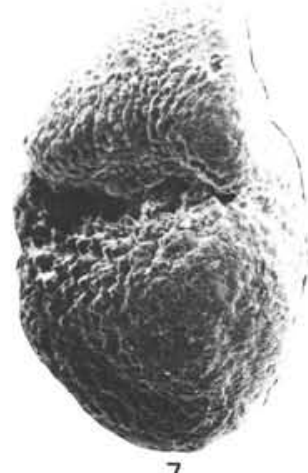

7

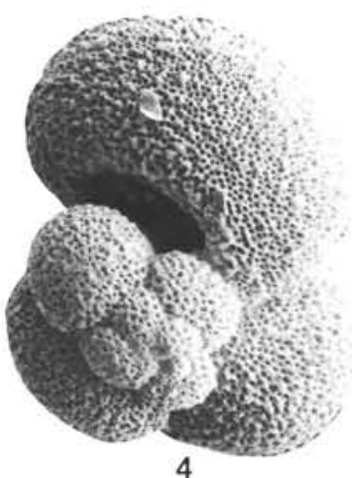

4
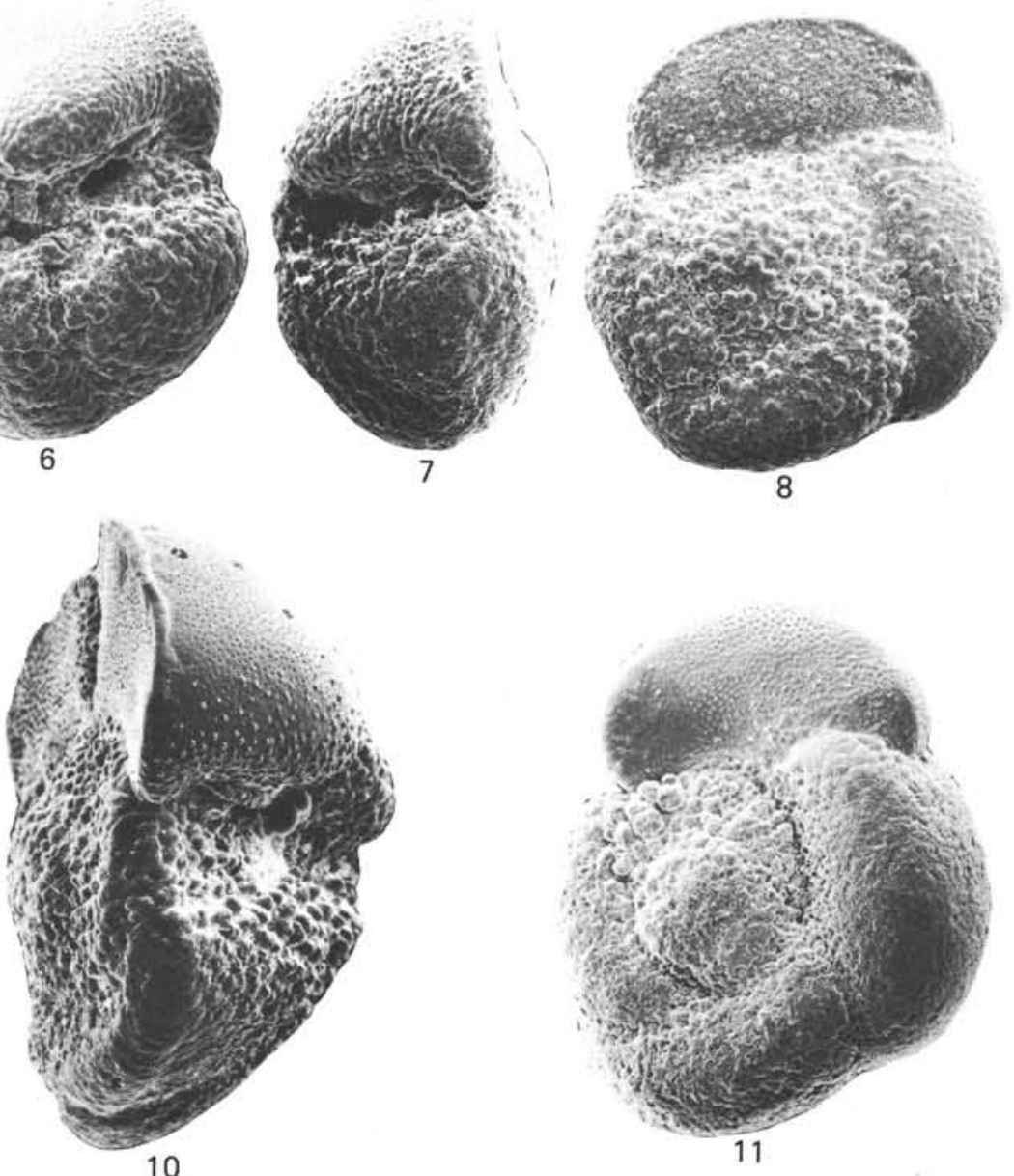

Plate 7. 1-3. Turborotalita humilis (Brady). (1) Sample 549A-1-1 $(1-4 \mathrm{~cm}), \times 200$, umbilical view, (2) Sample $549 \mathrm{~A}-1-1(1-4 \mathrm{~cm})$, $\times 200$, spiral view, (3) Sample 549A-1-1 (1-4 cm), $\times 200$, umbilical view. 4. Globigerina calida Parker, Sample 548-7-6 (110-117 cm), $\times 80$, spiral view. 5. Globigerinoides ruber (d'Orbigny), Sample 549-1-4 (40-43 cm), $\times 100$, spiral view. 6-8. Globorotalia crassaformis crassaformis (Galloway and Wissler). (6) Sample 549A-1-4 (10-13 cm), $\times 80$, umbilical view. (7) Sample 549A-1-4 (10-13 cm), $\times 80$, side view. (8) Sample 549A-1-4 (10-13 $\mathrm{cm}), \times 80$, spiral view. 9-11. Globorotalia crassaformis hessi Bolli and Premoli Silva. (9) Sample 549A-2-3 $(93-96 \mathrm{~cm})$, $\times 80$, umbilical view. (10) Sample 549A-2-3 (93-96 cm), $\times 80$, side view. (11) Sample 549A-3-3 $(132-134 \mathrm{~cm})$, $\times 80$, spiral view. 

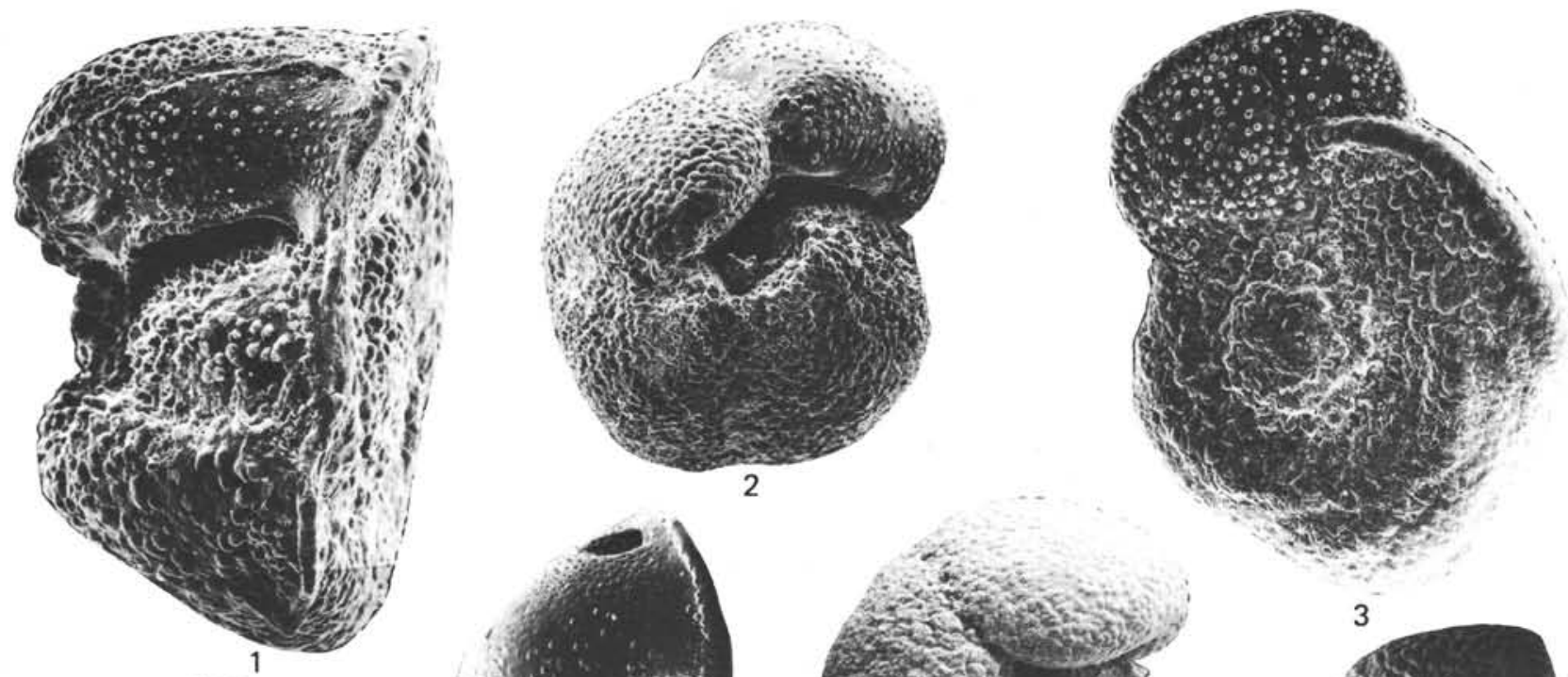

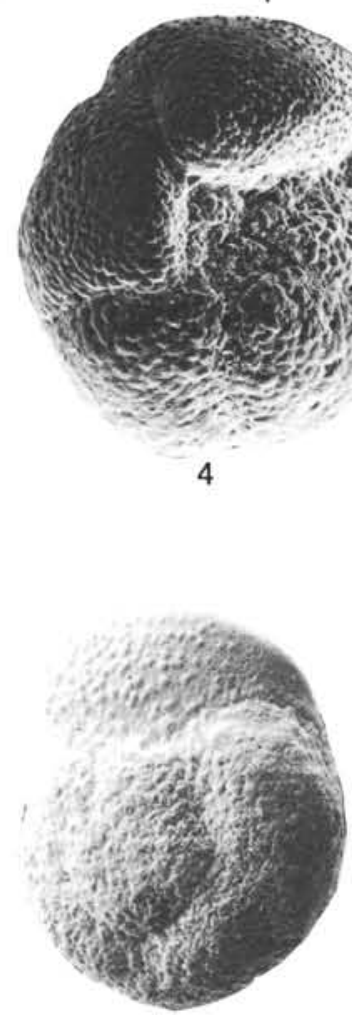

11

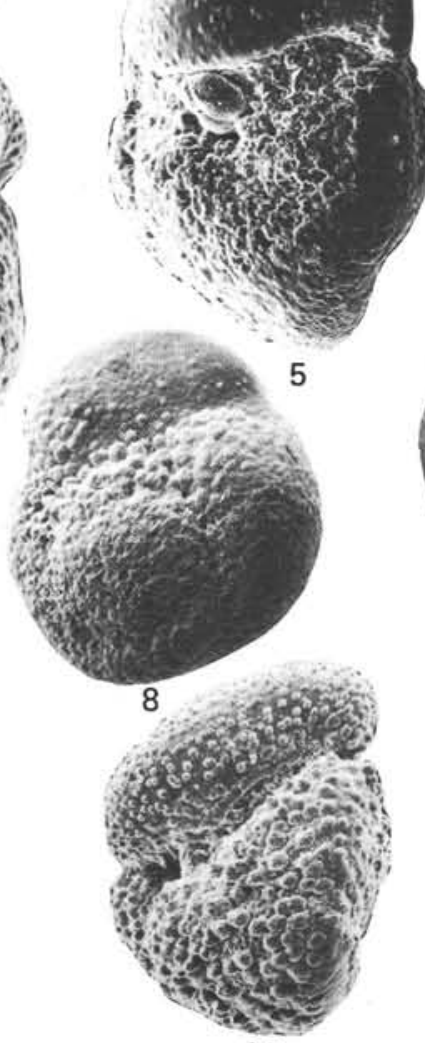

12

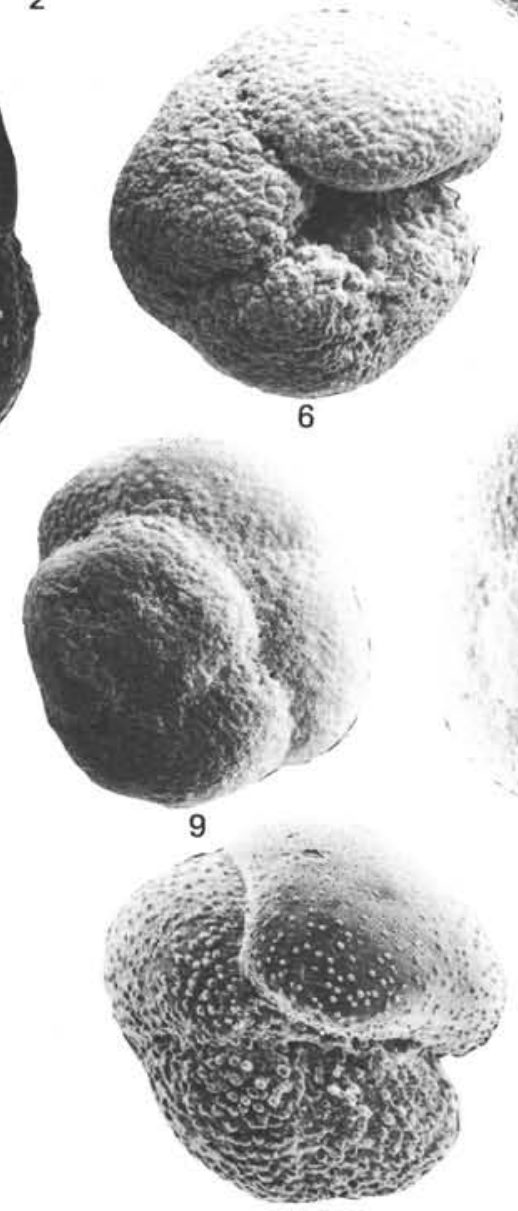

13
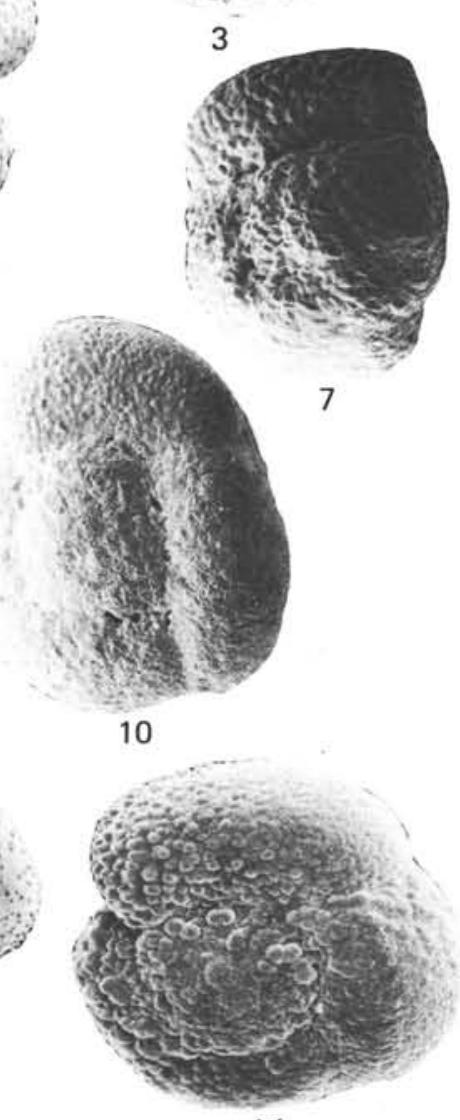

14

Plate 8. 1-5. Globorotalia crassaformis oceanica Cushman and Bermudez. (1) Sample $548-10, C C, \times 80$, side view. (2) Sample $548-10, C C, \times 60$, umbilical view. (3) Sample 548-10,CC, $\times 80$, spiral view. (4) Sample 548-10,CC, $\times 60$, umbilical view. (5) Sample 548-10,CC, $\times 80$, side view. 614. Globorotalia crassaformis ronda Blow. (6) Sample 548-11-1 (140-147 cm), $\times 80$, umbilical view. (7) Sample 548-9-2 (43-44 cm), $\times 80$, side view. (8) Sample 548-11-3 (139-146 cm), $\times 80$, spiral view. (9-10) Sample 548-9-2 (43-44 cm), $\times 80$, spiral view. (11) Sample 548-11-3 (139-146 $\mathrm{cm}$ ), $\times 80$, spiral view. (12) Sample 548-16,CC, $\times 100$, side view. (13) Sample 548-16,CC, $\times 100$, umbilical view. (14) Sample 548-16,CC, $\times 100$, spiral view. 

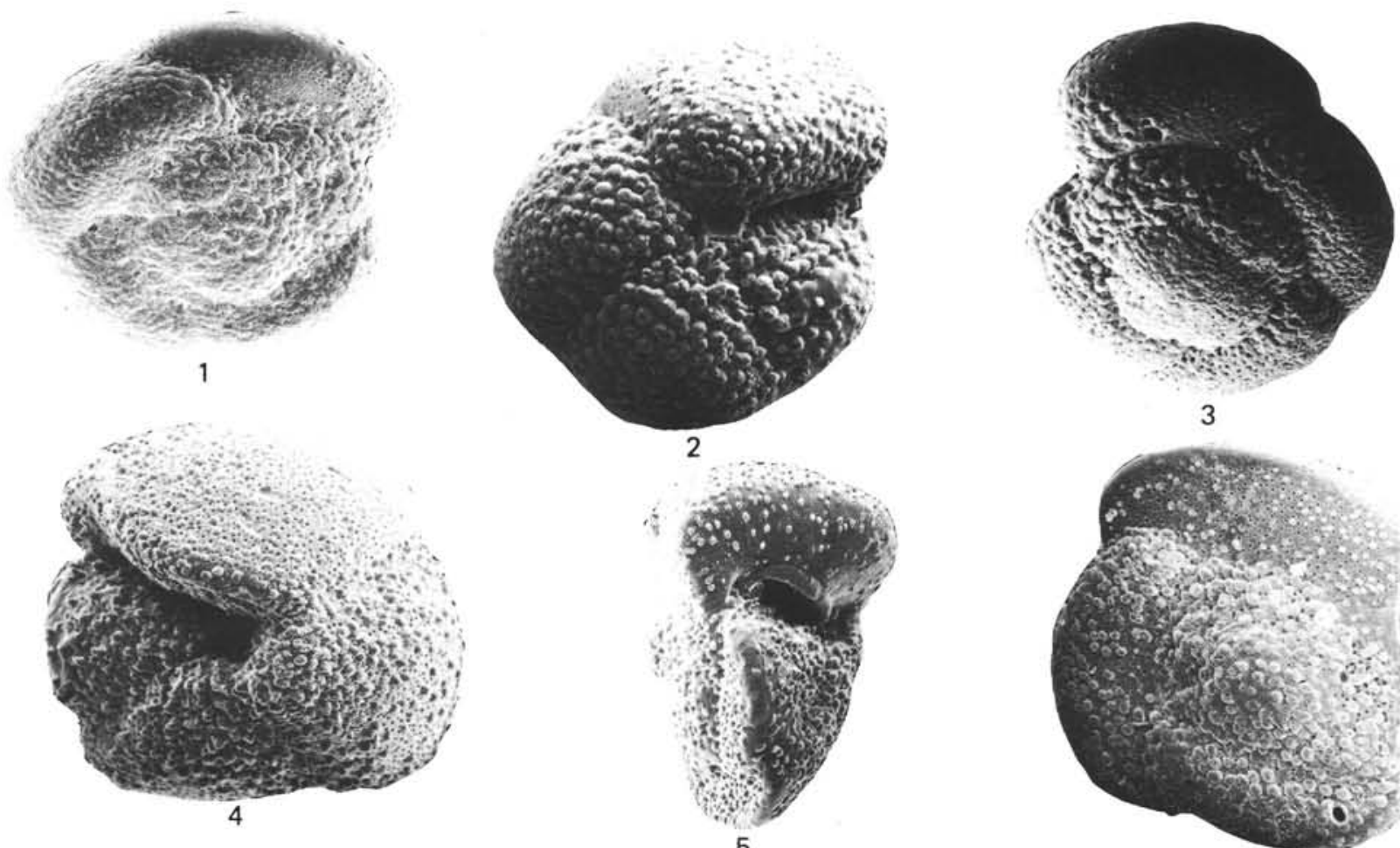

5
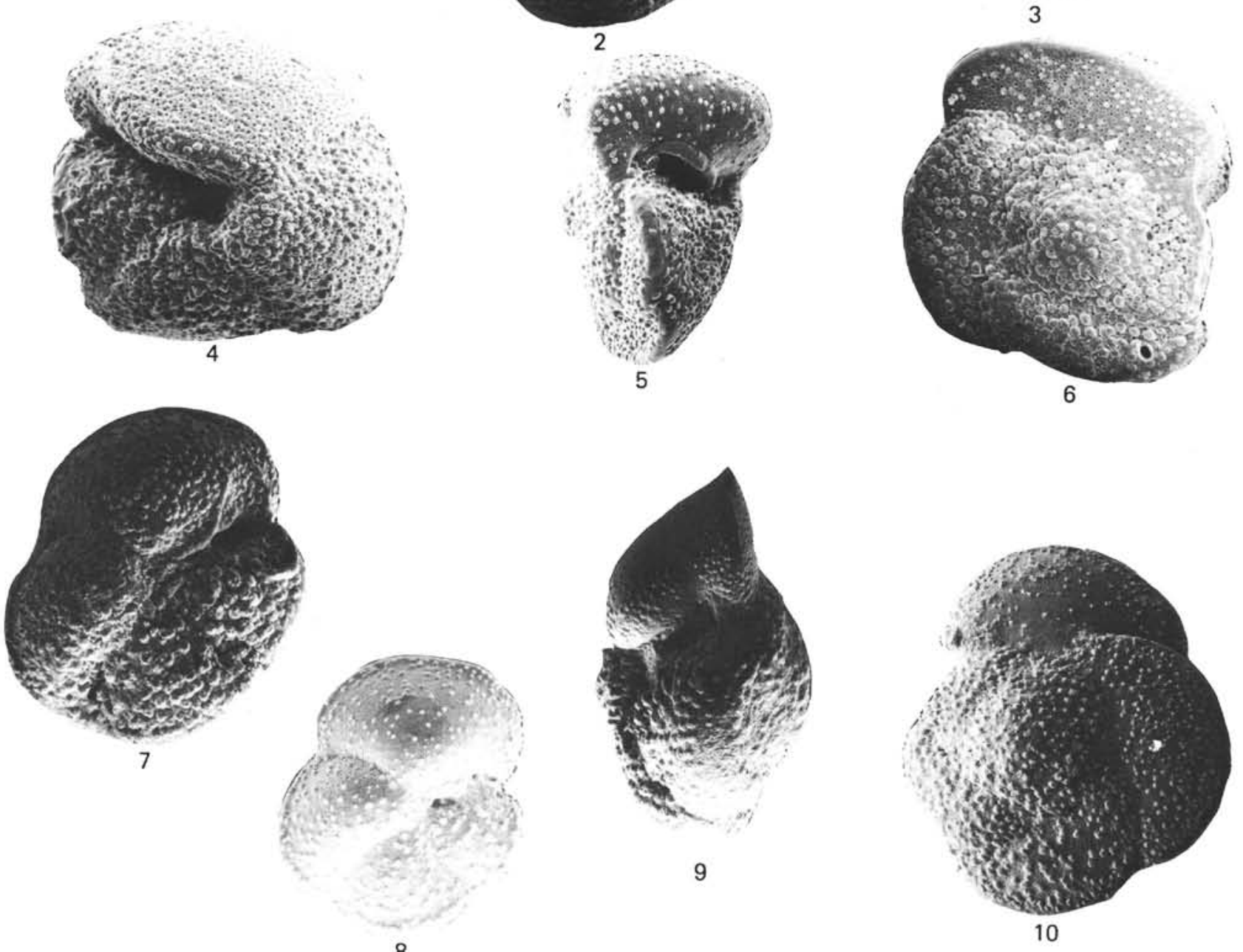

Plate 9. 1-3. Globorotalia crassaformis ronda Blow. (1) Sample 548-18,CC, $\times 100$, spiral view. (2) Sample 548-16-1 (70-77 cm), $\times 160$, umbilical view. (3) Sample 548-16-1 (70-77 cm), $\times 160$, spiral view. 4-6. Globorotalia crassaformis (Galloway and Wissler). (4) Sample 548-25,CC, $\times 100$, umbilical view. (5) Sample 548-25,CC, $\times 100$, side view. (6) Sample 548-25,CC, $\times 100$, spiral view. 7-10. Globorotalia crassula Cushman and Stewart. (7) Sample 548-15-5 (30-33 cm), $\times 100$, umbilical view. (8) Sample 548-15-5 (30-33 cm), $\times 100$, umbilical view. (9) Sample 548-15-5 (30$33 \mathrm{~cm}), \times 100$, side view. (10) Sample 548-15-5 $(30-33 \mathrm{~cm}), \times 80$, spiral view. 


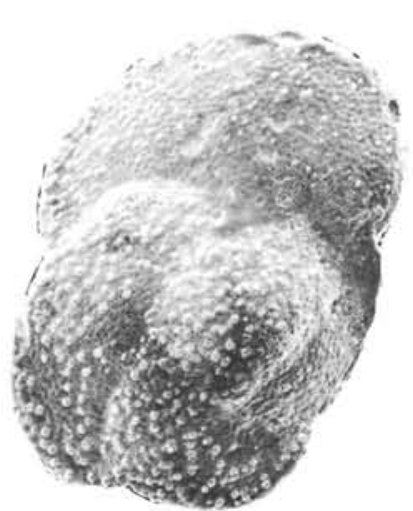

1

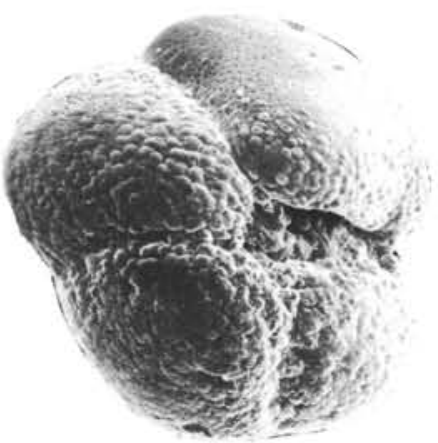

5

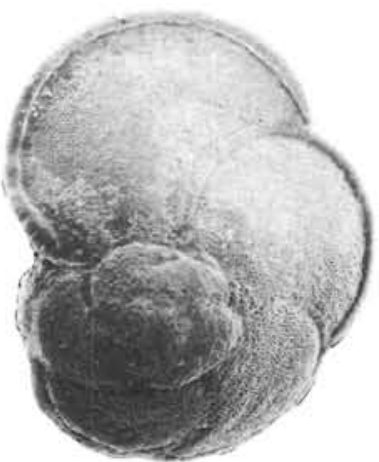

9
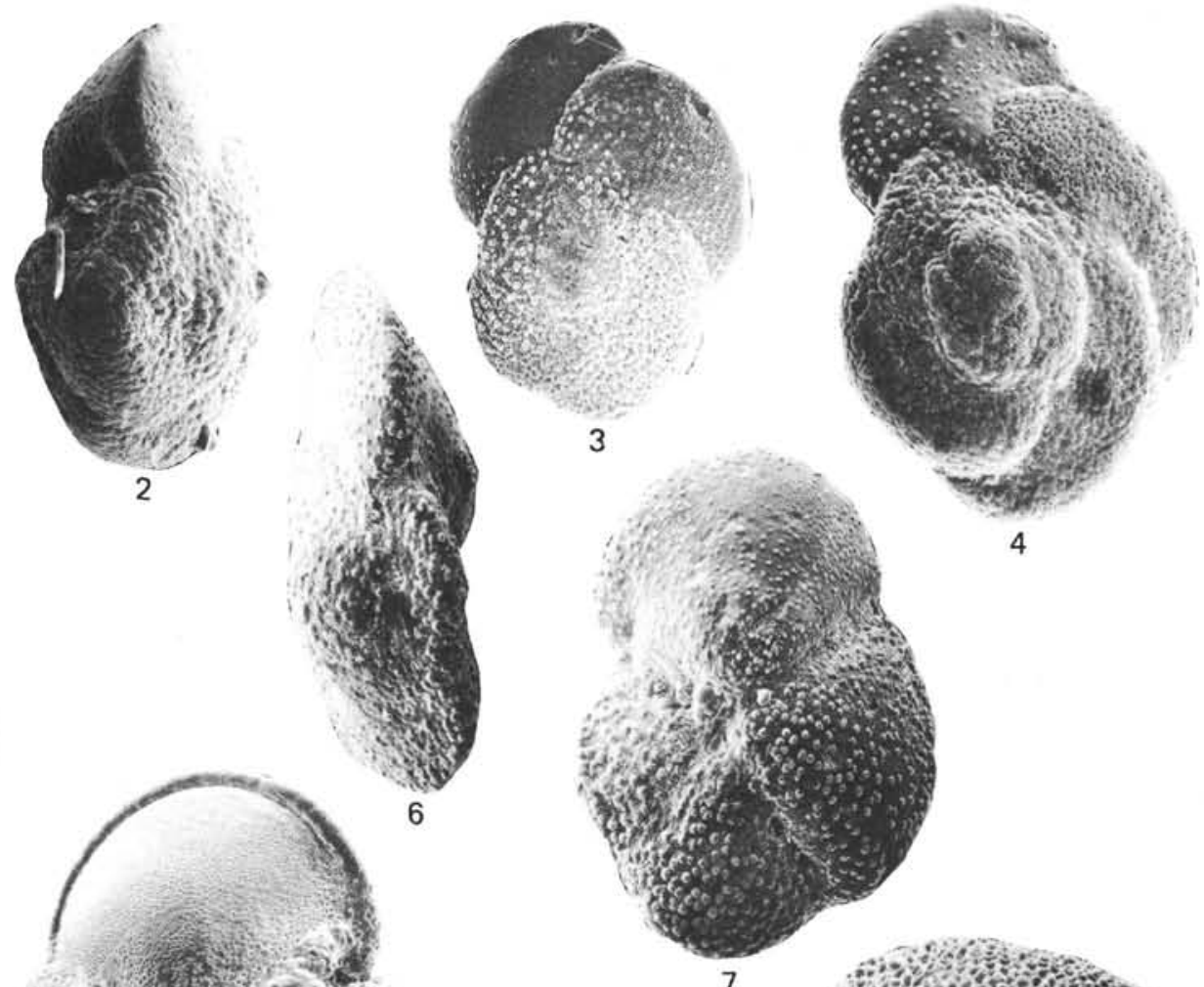

6

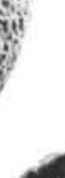

8

Plate 10. 1-7. Globorotalia crassula Cushman and Stewart. (1) Sample 548-29,CC, $\times 80$, spiral view. (2) Sample 548-21-3 (37-39 cm), $\times 80$, side view. (3) Sample 548-21-3 (37-39 cm), $\times 80$, spiral view. (4) Sample 548-21-3 (37-39 cm) $\times 80$, spiral view. (5) Sample 548-21-3 (37-39 cm), $\times 80$, umbilical view. (6) Sample 548-29,CC, $\times 100$, side view. (7) Sample 548-29,CC, $\times 80$, umbilical view. 8. Globorotalia tumida (Brady), Sample 549A-2-3 $(107-113 \mathrm{~cm}), \times 60$, umbilical view. 9. Globorotalia menardii (d'Orbigny), Sample 549A-2-3 $(107-113 \mathrm{~cm}), \times 60$, spiral view. 10 . Globorotalia $\mathrm{cf}$. flexuosa $($ Koch), Sample 549A-2-3 $(107-113 \mathrm{~cm}), \times 60$, umbilical view. 11. Globigerinoides conglobatus (Brady), Sample 549A-1-4 (40-43 cm), $\times 80$, spiral view. 

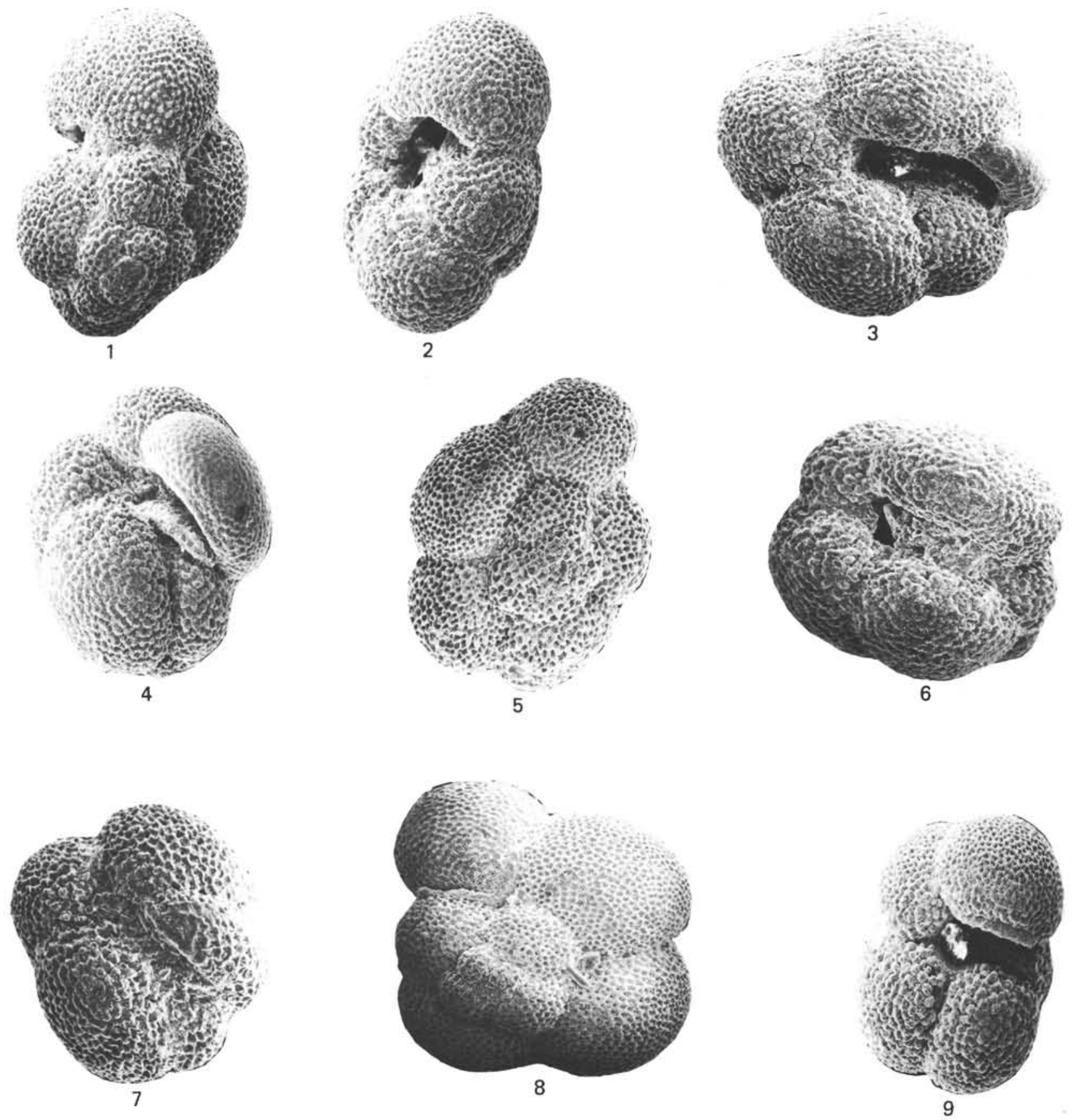

Plate 11. 1-9. Globigerina atlantica (Berggren). (1) Sample 548-18,CC, $\times 100$, spiral view. (2) Sample 548-28,CC, $\times 100$, side view. (3) Sample 548$25, \mathrm{CC}, \times 100$, umbilical view. (4) Sample 548-28,CC, $\times 100$, umbilical view. (5) Sample 548-28,CC, $\times 100$, spiral view. (6-7) Sample 548-18,CC, $\times 100$, umbilical view. (8) Sample 548-25,CC, $\times 100$, spiral view. (9) Sample 548-28,CC, $\times 100$, umbilical view. 


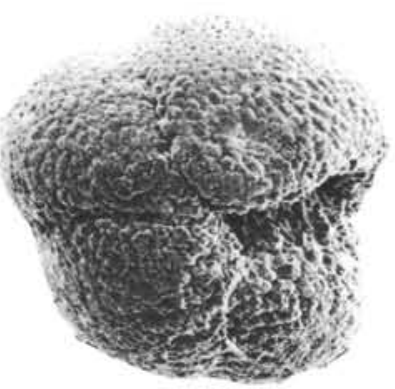

1

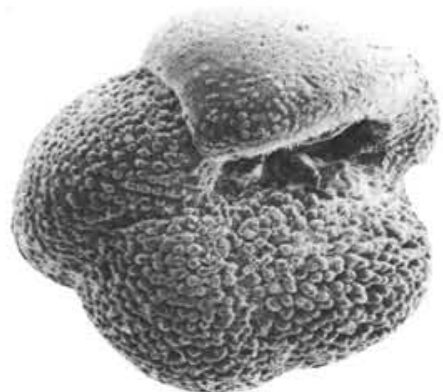

5

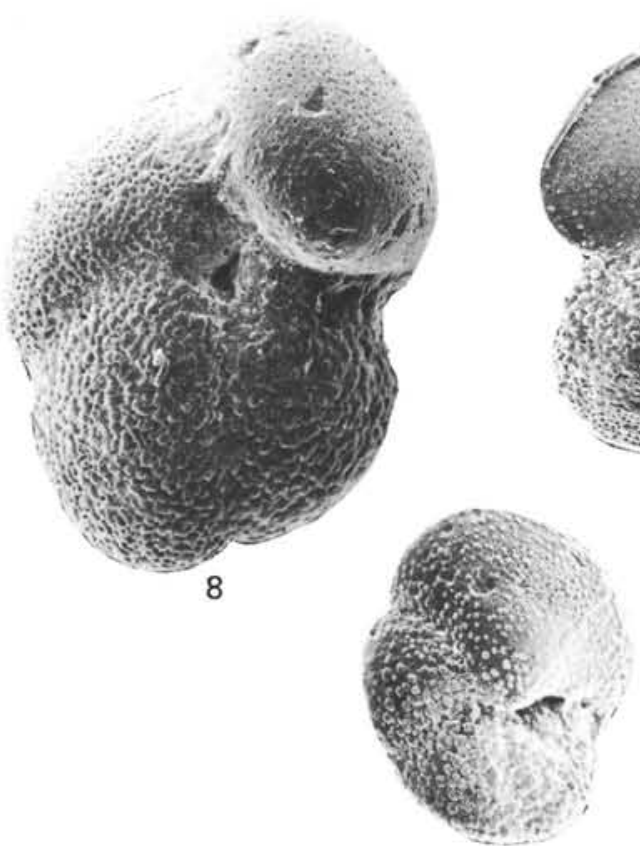

11

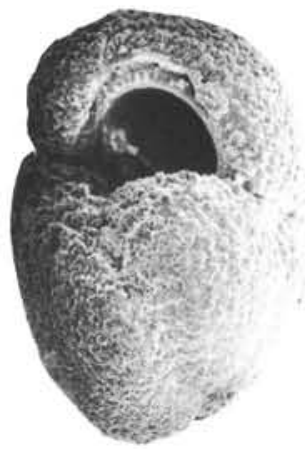

2

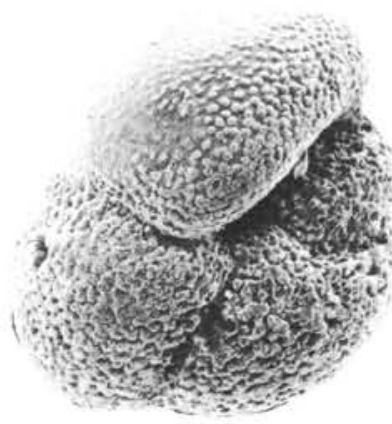

3
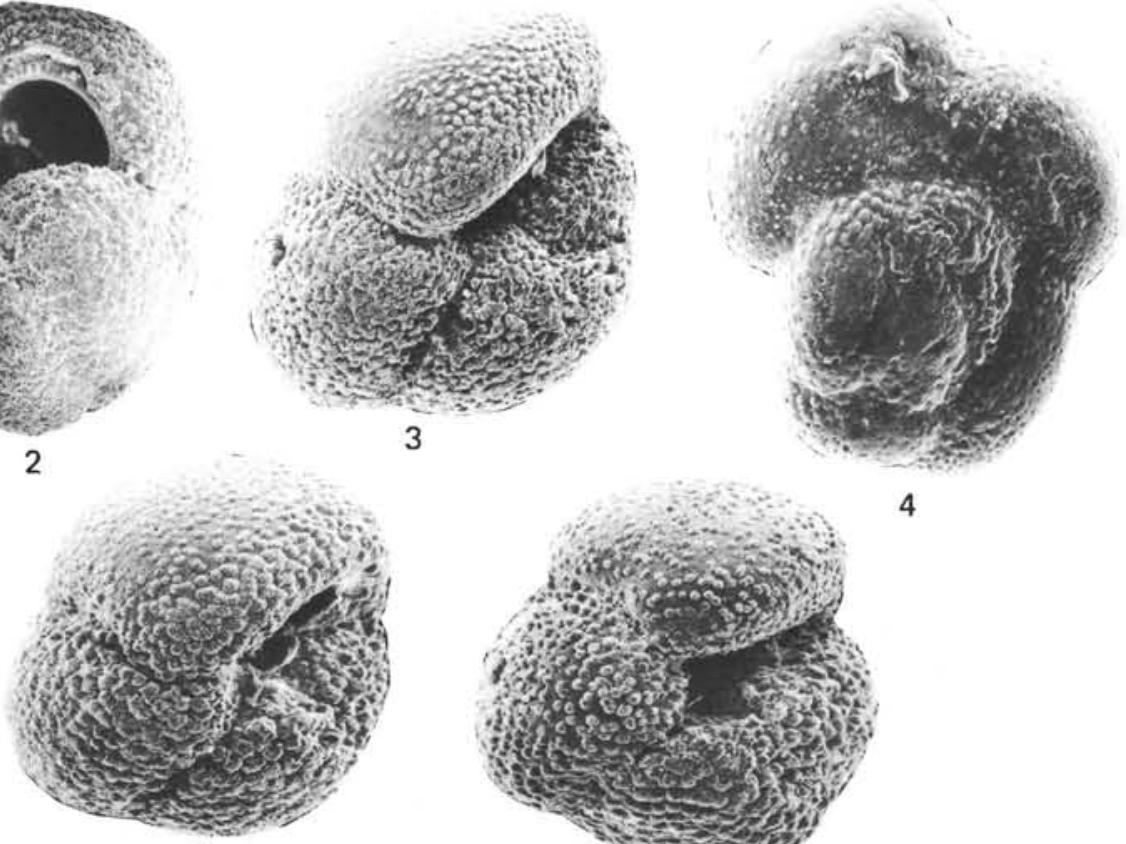

6

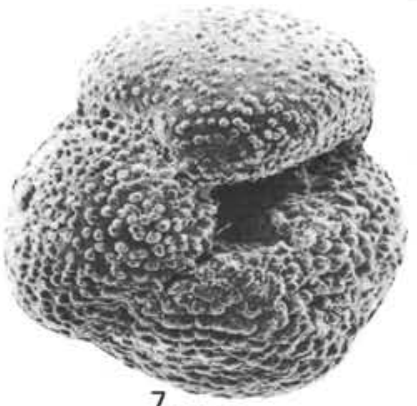

4
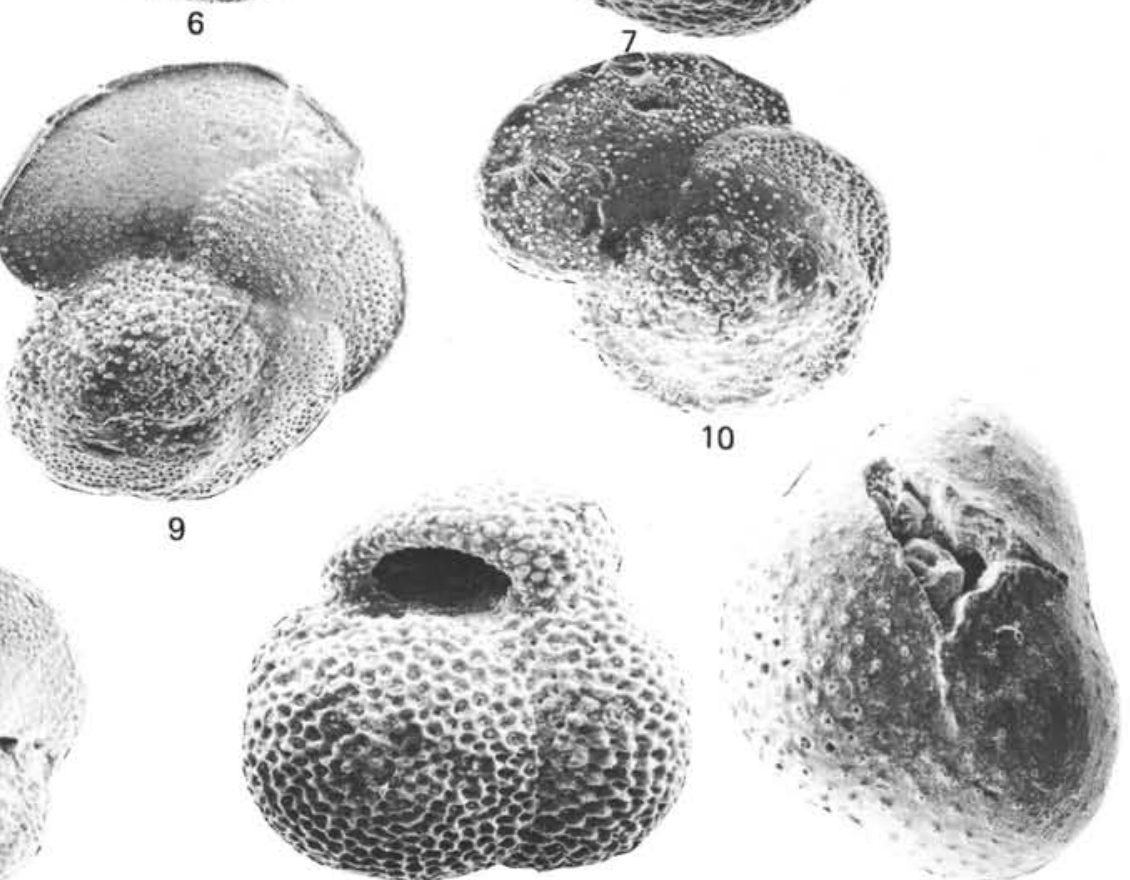

13

Plate 12. 1-8. Globorotalia puncticulata (Deshayes). (1) Sample 548-25,CC, $\times 100$, umbilical view. (2) Sample 548-25,CC, $\times 100$, side view. (3) Sample 548-32,CC, $\times 100$, umbilical view. (4) Sample 548-27,CC, $\times 100$, spiral view. (5-7) Sample 548-32,CC, $\times 100$, umbilical view. (8) Sample 548-29,CC, $\times 100$, umbilical view. 9-11. Globorotalia margaritae Bolli and Bermudez. (9-10) Sample 548-31,CC, $\times 100$, spiral view. (11) Sample 548-31,CC, $\times 100$, umbilical view. 12. Globigerinoides obliquus Bolli, Sample 548-35,CC, $\times 140$, umbilical view. 13. Sphaeroidinellopsis subdehiscens (Blow), Sample 548-35,CC, $\times 100$. 